\title{
Adicionando alças a uma superfície mínima
}

\section{Alexandre Lymberopoulos}

\author{
DiSSERTAÇÃO APRESENTADA
}

$\mathrm{AO}$

Instituto de Matemática e EstatísticA

DA

Universidade de SÃo Paulo

PARA

OBTENÇÃO DO GRAU

$\mathrm{DE}$

Mestre em Matemática

\section{Área de Concentração: GEOMETRIA \\ Orientador: Prof. Dr. Plínio Amarante Quirino Simões}

Durante a elaboração deste trabalho, o autor recebeu apoio financeiro da CAPES. 



\title{
Adicionando alças a uma superfície mínima
}

\author{
Este exemplar corresponde à redação \\ final da dissertação de mestrado devidamente \\ corrigida e defendida por Alexandre Lymberopoulos \\ e aprovada pela comissão julgadora.
}

São Paulo, novembro de 2003.

Banca examinadora:

- Prof. Dr. Plínio amarante Quirino Simões - IME-USP

- Prof. Dr. Cláudia Cueva Cândido - IME-USP

- Prof. Dr. Valério Ramos Batista - IMECC-UNICAMP 



\section{Agradecimentos}

Muitos são os que deveriam estar listados aqui. Em especial gostaria de agradecer: À banca examinadora, pelas enriquecedoras sugestões e considerações.

Ao Prof. Dr. Plínio Simões, pela paciência infindável, disposição e por tudo o que me ensinou desde os tempos do Cálculo I.

Ao Prof. Dr. Valério Ramos Batista, pela mesma paciência, disposição e grande ajuda ao longo deste trabalho.

Aos meus pais e irmãs, pelo suporte, estímulo e amor.

À Renata, meu amor, inspiração e vida.

Aos amigos Aritanan Gruber, Carlos Chacur (em memória), Célia Lopes, Daniel Tausk, Daniela Mariz, José Vilhena, Mario Leston, Roberto Masaishi, Rodnei, Rogério Brito, Sandro e muitos outros Cada um de vocês executou um papel memorável em minha vida.

Aos professores Cláudia Cândido, Luiz Augusto, Maria Elisa e Rosa Chaves.

À CAPES, instituição pela qual tive bolsa durante parte do trabalho. 



\section{Resumo}

Em 1989, H. Karcher [10] introduziu na teoria das superfícies mínimas do $\mathbb{R}^{3}$ a técnica de adicionar alças de diversos tipos a superfícies mínimas pré-existentes com a finalidade de obter novos exemplos de superfícies mínimas relevantes à teoria.

Neste trabalho descrevemos as técnicas usadas por M. Wohlgemuth em [16] para a adição de alças ao catenóide e apresentamos a construção de alguns exemplos de superfícies mínimas completas do $\mathbb{R}^{3}$ obtidos com esta técnica.

Fazendo uso dessa mesma técnica, descrevemos uma família de exemplos de superfícies mínimas em $\mathbb{R}^{3}$, originalmente construída por V. Ramos Batista (vide [1]), onde em cada família todo elemento tem gênero $n-1 \geq 1$ e todos os fins da superfície são catenoidais. Estes exemplos nos mostram que o grupo de simetrias junto com o tipo conforme não são suficientes para determinar a superfície mínima. 


\section{Abstract}

In 1989 H. Karcher [10] introduced a technique to add handles to known minimal surfaces in $\mathbb{R}^{3}$, to obtain new examples which are important to the theory.

On this work we describe the techniques used by M. Wohlgemuth in [16] to add handles to the catenoid. We also present the construction of examples of complete minimal surfaces in $\mathbb{R}^{3}$ using this technique.

Using the same method, we describe a family of examples of minimal surfaces in $\mathbb{R}^{3}$, originally constructed by $V$. Ramos Batista in [1], where each member of the family has genus $n-1 \geq 1$ and all of its ends are of catenoidal type. These examples show that the symmetry group and the conformal type are not enough to determine the minimal surface. 



\section{Sumário}

1 Superfícies Mínimas em $\mathbb{R}^{3} \quad 1$

1.1 Teoria básica de superfícies em $\mathbb{R}^{3} \ldots \ldots \ldots \ldots 1$

1.2 A segunda forma fundamental de uma superfície em $\mathbb{R}^{3} \ldots \ldots$. . . . 5

1.3 Superfícies de Riemann . . . . . . . . . . . . . . . . . . . . 11

1.4 Superfícies Mínimas e a representação de Weierstrass . . . . . . . . . 31

1.5 Princípios de simetria para superfícies mínimas . . . . . . . . . . 38

2 Obtendo novas superfícies a partir do catenóide 41

2.1 Catenóide . . . . . . . . . . . . . . . . . 41

2.2 Alças em uma superfície mínima. . . . . . . . . . . . . . 45

2.3 Exemplos ... . . . . . . . . . . . . . . 52

2.3.1 Catenóide duplamente periódico com quatro "alças de Schwarz" 52

2.3.2 Catenóide simplesmente periódico com quatro "alças de Schwarz" 55

2.3.3 Cerca de catenóides de gênero 2 . . . . . . . . . . 60

3 Birdcage-catenóides $\quad 65$

3.1 Equação algébrica do "birdcage" . . . . . . . . . . . . . . . 67

3.2 Os dados de Weierstrass para o "birdcage-catenóide" . . . . . . . . 71

3.3 O problema dos períodos . . . . . . . . . . . . . 75

Referências Bibliográficas $\quad 95$ 



\section{Lista de Figuras}

1.1 Vizinhança de um ponto de ramificação. . . . . . . . . . . . . . . 30

$2.1 M_{3}$-Costa-Hoffman-Meeks e o "birdcage-catenóide" de gênero 2. . . 42

2.2 O catenóide. . . . . . . . . . . . . . . . . . . . 42

2.3 Compactificação do catenóide. . . . . . . . . . . . . . . . . 42

2.4 Fatias do catenóide. . . . . . . . . . . . . . . . . 44

2.5 Alças de Schwarz numa superfície mínima. . . . . . . . . . . . . . 46

2.6 Alças de Neovius numa superfície mínima. . . . . . . . . . . . . . . . 46

2.7 Compatificação da cerca de catenóides. . . . . . . . . . . . . . . 47

2.8 A peça fundamental e as reflexões da cerca de catenóides. . . . . . . 47

2.9 Ângulos do vetor normal em uma $l_{j}$-alça. . . . . . . . . . . . . . . . . 52

2.10 Compactificação do catenóide duplamente periódico. . . . . . . . . 53

2.11 O catenóide duplamente periódico de gênero $2 \ldots \ldots$. . . . . . . . 53

2.12 Compactificação da cerca de catenóides simplesmente periódica com 4 alças. . . . . . . . . . . . . . . . . . 56

2.13 Cerca de catenóides simplesmente periódica com 4 alças. . . . . . . . 56

2.14 Cerca de catenóides de gênero 2 simplesmente periódico. . . . . . . . 61

2.15 Compactificação da cerca de catenóides de gênero 2. . . . . . . . . 61

3.1 O "birdcage" de gênero 2 e a $M_{3}$-Costa-Hoffman-Meeks. . . . . . . . 65

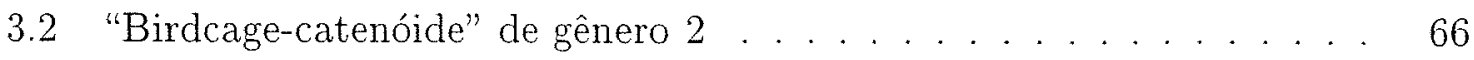

3.3 O "birdcage" e algumas curvas importantes. . . . . . . . . . . . . . 67

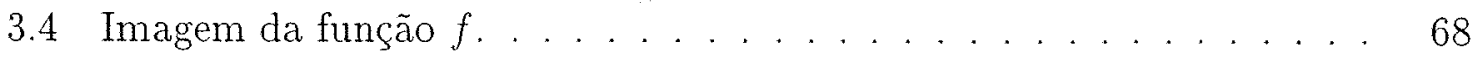

3.5 Simetria intrínseca do "birdcage". . . . . . . . . . . . . . 68

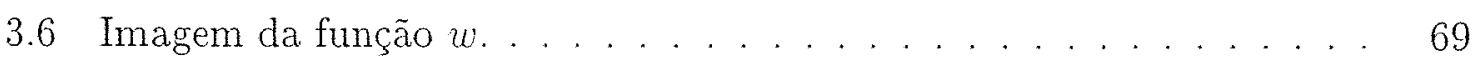

3.7 Zeros e pólos de $g, h$ e $w$ com suas multiplicidades. . . . . . . . . 72

3.8 Zeros e pólos de $d H$ com suas multiplicidades. . . . . . . . . . . . . 74

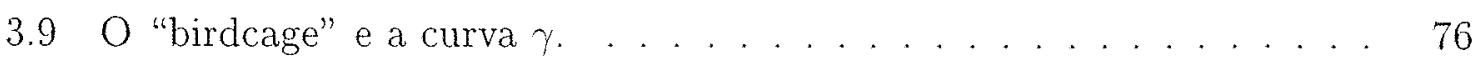

3.10 5-nóide. . . . . . . . . . . . . . . . . . . . . . . 88 
3.11 Zeros e pólos dos dados de Weierstrass do $n+2$-nóide. . . . . . . . 90

3.12 Setor circular e sua imagem por $G \ldots \ldots$. . . . . . . . . . . . 91

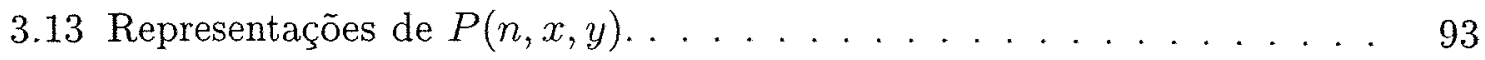




\section{Capítulo 1}

\section{Superfícies Mínimas em $\mathbb{R}^{3}$}

Neste capítulo são apresentados resultados necessários para o desenvolvimento do trabalho. Tratamos da teoria básica de superfícies, alguns resultados sobre superfícies de Riemann, representação de Weierstrass e dos princípios de simetria para superfícies mínimas.

\subsection{Teoria básica de superfícies em $\mathbb{R}^{3}$}

Definição 1.1.1. Sejam $M$ uma 2-variedade $C^{\infty}$ conexa orientável e $f: M \hookrightarrow \mathbb{R}^{3}$ uma imersão. Então, $S$ é uma superfície em $\mathbb{R}^{3}$ se existirem $M$ e $f$ nas condições acima satisfazendo $S=f(M)$.

Vamos dar a $M$ uma métrica Riemanniana fazendo o "pull-back" por $f$ da métrica usual do $\mathbb{R}^{3}$, ou seja, dados $u, v \in T_{p} M$ definimos

$$
d s^{2}(u, v):=f^{*}\langle u, v\rangle=\langle d f(u), d f(v)\rangle
$$

Assim $\left(M, d s^{2}\right)$ é uma 2-variedade Riemanniana. Sejam então $\nabla$ a conexão de Levi-Civita de $d s^{2}, \Sigma(M)$ sua estrutura diferenciável e $\bar{\nabla}$ a conexão de Levi-Civita do produto interno canônico, $\langle$,$\rangle , no \mathbb{R}^{3}$.

Dizemos que $M$ é completa com respeito a $d s^{2}$ se $\forall \gamma:[0, a[\rightarrow M$ caminho divergente em $M$ temos $\int_{0}^{a}\left|\gamma^{\prime}(t)\right| d t=\infty$, onde $\left|\gamma^{\prime}(t)\right|^{2}=d s^{2}\left(\gamma^{\prime}(t), \gamma^{\prime}(t)\right)$. A superfície $S=f(M)$ é completa se $M$ for completa relativamente à métrica induzida por $f$ sobre $M$.

Vamos a partir de agora identificar $\mathbb{R}^{2}$ com $\mathbb{C}$ associando ao par $(u, v) \in \mathbb{R}^{2}$ o complexo $u+i v$. 
Definição 1.1.2. Sejam $(U, w=u+i v) \in \Sigma(M), S=f(M)$ uma superfície, $f_{u}:=$ $\frac{\partial}{\partial u}\left(f \circ w^{-1}\right)$ e $f_{v}:=\frac{\partial}{\partial v}\left(f \circ w^{-1}\right)$. Então, $(U, w=u+i v)$ é um sistema de coordenadas isotérmicas para $S$ se existe $\lambda \in C^{\infty}(w(U)), \lambda>0$ tal que

$$
\lambda^{2}=\left\langle f_{u}, f_{u}\right\rangle=\left\langle f_{v}, f_{v}\right\rangle ; \quad\left\langle f_{u}, f_{v}\right\rangle \equiv 0
$$

Nessas condições temos que $d s^{2}=\lambda^{2}\left(d u^{2}+d v^{2}\right)$ ao longo de $U$. O teorema a seguir garante a existência de coordenadas isotérmicas para todas as superfícies de classe $C^{2}$.

Teorema 1.1.3. Seja $U$ aberto conexo de $\mathbb{C}$ e $f: U \hookrightarrow \mathbb{R}^{3}$ uma imersão de classe $C^{2}$. Então $\forall w_{0} \in U$ existem $V \subset U$, aberto, com $w_{0} \in V$ e $\phi: V \rightarrow \phi(V) \subset \mathbb{C}$ difeomorfismo de classe $C^{2}$ tal que $(V, \phi)$ é um sistema de coordenadas isotérmicas relativo à $f(U)$.

Demonstração: Ver [14].

$\mathrm{O}$ atlas constituído por coordenadas isotérmicas positivas (isto é, todas as mudanças de coordenadas têm Jacobiano positivo) cobre $M$ e induz uma estrutura holomorfa $\mathcal{H}(M)$ sobre $M$ tornando-a uma superfície de Riemann. A seção 1.3 traz a definição e alguns resultados importantes sobre estas superfícies.

Mais formalmente:

Definição 1.1.4. Seja $\Sigma(M)$ a estrutura diferenciável de $M$. O atlas $\mathcal{A}(M) \subset \Sigma(M)$ é uma estrutura holomorfa de $M$ se para todo $p \in M$ existe $(U, \phi) \in \mathcal{A}(M)$ tal que $p \in U$ e para todos $(U, \phi),(V, \psi) \in \mathcal{A}(M) \operatorname{com} U \cap V \neq \emptyset$ temos que

$$
\psi \circ \phi^{-1}: \phi(U \cap V) \rightarrow \psi(U \cap V) \text { e } \phi \circ \psi^{-1}: \psi(U \cap V) \rightarrow \phi(U \cap V)
$$

são funções complexas holomorfas.

O teorema seguinte é crucial para o que se segue no trabalho, pois trataremos de imersões mínimas de superfícies de Riemann compactas menos um número finito de pontos.

Teorema 1.1.5. Se $S=f(M)$ é uma superfície do $\mathbb{R}^{3}$ e $\mathcal{H}(M) \subset \Sigma(M)$ é o conjunto dos sistemas de coordenadas isotérmicas positivas de $M$ então $\mathcal{H}(M)$ é uma estrutura holomorfa de $M$. Assim, $(M, \mathcal{H}(M))$ é uma superfície de Riemann.

\section{Demonstração:}

$\operatorname{Sejam}(U, w=u+i v),(V, z=x+i y) \in \mathcal{H}(M), \operatorname{com} U \cap V \neq \emptyset$.

Vamos mostrar que $z \circ w^{-1}: w(U \cap V) \rightarrow z(U \cap V)$ é holomorfa, ou seja, $z(w)=$ $x(u, v)+i y(u, v)$ satisfaz as equações de Cauchy-Riemann: 


$$
x_{u}=y_{v} ; \quad x_{v}=-y_{u}, \text { sobre } U \cap V .
$$

Sejam $\lambda \in C^{\infty}(w(U))$ e $\mu \in C^{\infty}(z(V))$ funções positivas dadas pelos sistemas de coordenadas isotérmicos, isto é, $d s^{2}=\lambda^{2}\left(d u^{2}+d v^{2}\right)$ ao longo de $U$ e $d s^{2}=$ $\mu^{2}\left(d x^{2}+d y^{2}\right)$ ao longo de $V$. Então, ao longo de $U \cap V$ temos $d s^{2}=\lambda^{2}\left(d u^{2}+d v^{2}\right)=$ $\mu^{2}\left(d x^{2}+d y^{2}\right)$.

Porém,

$$
\begin{aligned}
d x & =x_{u} d u+x_{v} d v \mathrm{e} \\
d y & =y_{u} d u+y_{v} d v
\end{aligned}
$$

donde

$$
\begin{aligned}
& d x^{2}=d x \otimes d x=x_{u}^{2} d u^{2}+x_{u} x_{v} d u \otimes d v+x_{u} x_{v} d v \otimes d u+x_{v}^{2} d v^{2} \mathrm{e} \\
& d y^{2}=d y \otimes d y=y_{u}^{2} d u^{2}+y_{u} y_{v} d u \otimes d v+y_{u} y_{v} d v \otimes d u+y_{v}^{2} d v^{2}
\end{aligned}
$$

E então

$$
\begin{aligned}
\lambda^{2}\left(d u^{2}+d v^{2}\right) & \equiv \mu^{2}\left(d x^{2}+d y^{2}\right) \\
& =\mu^{2}\left(\left(x_{u}^{2}+y_{u}^{2}\right) d u^{2}+2\left(x_{u} x_{v}+y_{u} y_{v}\right) d u \otimes d v+\left(x_{v}^{2}+y_{v}^{2}\right) d v^{2}\right)
\end{aligned}
$$

Logo,

$$
\begin{aligned}
\lambda^{2} & \equiv \mu^{2}\left(x_{u}^{2}+y_{u}^{2}\right), \\
\lambda^{2} & \equiv \mu^{2}\left(x_{v}^{2}+y_{v}^{2}\right), \text { e } \\
x_{u} x_{v}+y_{u} y_{v} & \equiv 0 \text { ao longo de } U \cap V .
\end{aligned}
$$

De (1.4) temos $\left(x_{u}, y_{u}\right) \equiv \rho\left(-y_{v}, x_{v}\right)$ sobre $U \cap V$.

As equações (1.3) e (1.4) implicam $x_{u}^{2}+x_{v}^{2} \equiv x_{v}^{2}+y_{v}^{2}$. Disto e da conclusão acima temos $\rho= \pm 1$.

Mas se $\left\{\partial_{u}, \partial_{v}\right\}$ e $\left\{\partial_{x}, \partial_{y}\right\}$ são respectivamente as bases duais de $\{d u, d v\}$ e $\{d x, d y\}$ para $T_{p} w(U \cap V)$ temos

$$
\begin{aligned}
& \partial_{u}=x_{u} \partial_{x}+y_{u} \partial_{y} \\
& \partial_{v}=x_{v} \partial_{x}+y_{v} \partial_{y}
\end{aligned}
$$


Como $\left\{\partial_{u}, \partial_{v}\right\}$ e $\left\{\partial_{x}, \partial_{y}\right\}$ possuem a mesma orientação temos que $d u \wedge d v\left(\partial_{x}, \partial_{y}\right)>$ 0. Porém:

$$
\begin{aligned}
d u \wedge d v\left(\partial_{u}, \partial_{v}\right) & =d u\left(\partial_{u}\right) d v\left(\partial_{v}\right)-d u\left(\partial_{v}\right) d v\left(\partial_{u}\right) \\
& =\left[x_{u} d u\left(\partial_{x}\right)+y_{u} d u\left(\partial_{y}\right)\right]\left[x_{v} d v\left(\partial_{x}\right)+y_{v} d v\left(\partial_{y}\right)\right] \\
& -\left[x_{v} d u\left(\partial_{x}\right)+y_{v} d u\left(\partial_{y}\right)\right]\left[x_{u} d v\left(\partial_{x}\right)+y_{u} d v\left(\partial_{y}\right)\right] \\
& =\left(x_{u} y_{v}-x_{v} y_{u}\right) d u(\partial x) d v(\partial y)-\left(y_{v} x_{u}-y_{u} x_{v}\right) d u(\partial y) d v(\partial x) \\
& =\left(x_{u} y_{v}-x_{v} y_{u}\right)[d u(\partial x) d v(\partial y)-d u(\partial y) d v(\partial x)] \\
& =\left|\begin{array}{ll}
x_{u} & y_{u} \\
x_{v} & y_{v}
\end{array}\right|
\end{aligned}
$$

$\operatorname{Logo}\left|\begin{array}{ll}x_{u} & y_{u} \\ x_{v} & y_{v}\end{array}\right|>0$. Como

$$
0<x_{u} y_{v}-x_{v} y_{u}=-\rho y_{v}^{2}-\rho y_{u}^{2}=-\rho\left(y_{u}^{2}+y_{v}^{2}\right)
$$

então $\rho \equiv-1$ e portanto

$$
x_{u} \equiv y_{v} \text { e } x_{v} \equiv-y_{u} .
$$

A partir de agora vamos supor que $M$ é uma superfície de Riemann e nos referiremos a $S=f(M)$ como uma superfície conforme do $\mathbb{R}^{3}$.

Definição 1.1.6. Se $h \in C^{\infty}(M)$ e $(U, w=u+i v) \in \mathcal{H}(M)$, sejam

$$
h_{u}:=\frac{\partial}{\partial u}\left(h \circ w^{-1}\right) ; h_{v}:=\frac{\partial}{\partial v}\left(h \circ w^{-1}\right) ; h_{w}:=\frac{1}{2}\left(h_{u}-i h_{v}\right) ; h_{\bar{w}}:=\frac{1}{2}\left(h_{u}+i h_{v}\right) .
$$

Proposição 1.1.7. Se $h \in C^{\infty}(M, \mathbb{C})$ então

(a) $h$ é holomorfa $\Longleftrightarrow h_{\bar{w}} \equiv 0, \forall(U, w=u+i v) \in \mathcal{H}(M)$.

(b) $h_{\bar{w} w}=h_{w \bar{w}}=\frac{1}{4}\left(h_{u u}+h_{v v}\right)$.

\section{Demonstração:}

(a) Suponha que $h \circ w^{-1}(w(p))=\left(h \circ w^{-1}\right)_{1}(w(p))+i\left(h \circ w^{-1}\right)_{2}(w(p))=h_{1}(w(p))+$ $i h_{2}(w(p))$. Para que $h$ seja holomorfa ela deve satisfazer as equações de CauchyRiemann:

$$
\left(h_{1}\right)_{u}=\left(h_{2}\right)_{v} ; \quad\left(h_{1}\right) v=-\left(h_{2}\right)_{u} .
$$

Se estas equações valem, então 


$$
\begin{aligned}
h_{\bar{w}} & =\frac{1}{2}\left(h_{u}+i h_{v}\right) \\
& =\frac{1}{2}\left(\left(h_{1}\right)_{u}+i\left(h_{2}\right)_{u}+i\left(\left(h_{1}\right)_{v}+i\left(h_{2}\right)_{v}\right)\right) \\
& =\frac{1}{2}\left(\left(h_{1}\right)_{u}-\left(h_{2}\right)_{v}+i\left(\left(h_{2}\right)_{u}-\left(h_{1}\right)_{v}\right)\right)=0 .
\end{aligned}
$$

Reciprocamente, se $h_{\bar{w}} \equiv 0$, então

$$
\begin{aligned}
0 & =h_{\bar{w}} \\
& =h_{u}+i h_{v} \\
& =\left(h_{1}\right)_{u}+i\left(h_{2}\right)_{u}+i\left(\left(h_{1}\right)_{v}+i\left(h_{2}\right)_{2}\right) \\
& =\left(\left(h_{1}\right)_{u}-\left(h_{2}\right)_{v}\right)+i\left(\left(h_{1}\right)_{v}+\left(h_{2}\right)_{u}\right)
\end{aligned}
$$

donde $\left(h_{1}\right)_{u}=\left(h_{2}\right)_{v}$ e $\left(h_{1}\right)_{v}=-\left(h_{2}\right)_{u}$.

(b)

$$
h_{w \bar{w}}=\left(\frac{1}{2}\left(h_{u}-i h_{v}\right)\right)_{\bar{w}}=\frac{1}{2}\left(\frac{1}{2}\left(h_{u u}+i h_{u v}\right)-\frac{i}{2}\left(h_{v u}+i h_{v v}\right)\right)=\frac{1}{4}\left(h_{u u}+h_{v v}\right) .
$$

Uma conta semelhante mostra a primeira igualdade.

\subsection{A segunda forma fundamental de uma super- fície em $\mathbb{R}^{3}$}

Faremos nesta seção o desenvolvimento da segunda forma fundamental de uma superfície. A teoria apresentada aqui tem caráter bem mais geral do que a necessária para as superfícies em $\mathbb{R}^{3}$.

Sejam $S=f(M)$ uma superfície do $\mathbb{R}^{3}, \nabla$ a conexão de Levi-Civita de $M$ e $\bar{\nabla}$ a de $\mathbb{R}^{3}$. Seja ainda $\mathscr{X}(f)$ o conjunto dos campos de vetores de classe $C^{\infty}$ em $\mathbb{R}^{3}$ ao longo de $S=f(M)$, isto é, $X \in \mathscr{X}(f)$ se $X: M \rightarrow T \mathbb{R}^{3}$ satisfaz $X(p) \in T_{f(p)} \mathbb{R}^{3}$, para todo $p \in M$ e para toda $h \in C^{\infty}(M)$ a aplicação de $M$ em $\mathbb{R}$ dada por $p \mapsto$ $X(p)[h]:=d h(X(p))$ é de classe $C^{\infty}$.

Observações:

1. $f_{*}:=d f, \forall f \in C^{\infty}\left(M, \mathbb{R}^{3}\right)$. Ambos os símbolos serão usados ao longo do texto. 
2. Se $X \in \mathscr{X}(f)$, escrevemos $X=X^{T}+X^{\perp}$, que são respectivamente as componentes tangencial e normal de $X$ relativamente à $S=f(M)$.

As conexões $\nabla$ e $\bar{\nabla}$ nos permitem definir o seguinte operador sobre $\mathscr{X}(f)$ :

$$
\begin{aligned}
\nabla^{f}: \mathscr{X}(M) \times \mathscr{X}(f) & \rightarrow \mathscr{X}(f) \\
(X, Y) & \rightarrow \nabla_{X}^{f} Y \text { dado por } \nabla_{X}^{f} Y:=\bar{\nabla}_{d f\left(X_{p}\right)} \tilde{Y}
\end{aligned}
$$

onde $\tilde{Y}$ é qualquer estensão $C^{\infty}$ de $Y$ a uma vizinhança aberta de $f(p)$ em $\mathbb{R}^{3}$ e $\mathscr{X}(M)$ é o conjunto dos campos de vetores de classe $C^{\infty}$ em $M$.

Esse operador tem as seguintes propriedades para $X \in \mathscr{X}(M)$ e $Y, Z \in \mathscr{X}(f)$ :

1. Se $h \in C^{\infty}(M)$ então $\nabla_{h X}^{f}(Y+Z)=h \nabla_{X}^{f} Y+h \nabla_{X}^{f} Z$

2. Se $h \in C^{\infty}(M)$ então $\nabla_{X}^{f} h Y=X[h] Y+h \nabla_{X}^{f} Y, X[h]:=d h(X)$

3. Se $X, Y \in \mathscr{X}(M)$ então $f_{*}\left(\nabla_{X} Y\right)=\left(\nabla_{X}^{f} f_{*}(Y)\right)^{T}$.

Se $X, Y \in \mathscr{X}(M)$ podemos escrever $\nabla_{X}^{f} Y$ querendo dizer $\nabla_{X}^{f} f_{*}(Y)$.

A partir de $\nabla^{f}$ definimos a segunda forma fundamental de $S$ (ou de $f$ ), indicada por $B(X, Y)$. Para $X, Y \in \mathscr{X}(M)$ :

$$
B(X, Y):=\left(\nabla_{X}^{f} Y\right)^{\perp}=\nabla_{X}^{f} Y-\left(\nabla_{X}^{f} Y\right)^{T}=\nabla_{X}^{f} Y-f_{*}\left(\nabla_{X} Y\right)
$$

Considerando agora a derivada covariante do tensor $f_{*}\left(f_{*}\right.$ é um 1-tensor covariante a valores em $\mathscr{X}(f))$ temos

$$
\left(\nabla_{X} f_{*}\right)(Y)=\bar{\nabla}_{f_{*}(X)} f_{*}(Y)-f_{*}\left(\nabla_{X} Y\right)
$$

Se definirmos $\left(\nabla f_{*}\right)(X, Y):=\left(\nabla_{X} f_{*}\right)(Y)$ então $B=\nabla f_{*}$, isto é, $B$ mede a variação do plano tangente, ou seja, o modo como $M$ está imersa em $\mathbb{R}^{3}$. Desta observação vemos que $B$ é um objeto da geometria extrínseca de $M$, isto é, $B$ depende do espaço ambiente da imersão $f$. Um exemplo disso na teoria de superfícies mínimas é o caso do catenóide menos uma catenária e de um período do helicóide. As duas superfícies são isométricas e são imersões da esfera de Riemann menos um meridiano com esboços completamente diferentes em $\mathbb{R}^{3}$.

Proposição 1.2.1. B tem caráter tensorial e é simétrica. 
Demonstração: $B(X, Y)-B(Y, X)=\nabla_{X}^{f} Y-\nabla_{Y}^{f} X-f_{*}\left(\nabla_{X} Y\right)+f_{*}\left(\nabla_{Y} X\right)=$ $f_{*}([X, Y])-f_{*}([X, Y]) \equiv 0$. Portanto. $B$ é simétrica e da definição vemos que é linear na primeira coordenada. Logo, $B$ é bilinear e simétrica.

Como $B(X, Y) \in \mathscr{X}(f)^{\perp}$ então para $p \in M$ e $u, v \in T_{p} M$ determinamos $B_{p}(u, v)$ se calculamos $\left\langle B_{p}(u, v), w\right\rangle, \forall w \in T_{f(p)} S^{\perp}$. Assim, podemos definir para cada $Z \in$ $\mathscr{X}(f)^{\perp}$ o operador $A^{Z}: \mathscr{X}(M) \rightarrow \mathscr{X}(M)$ como a única soluçào de

$$
d s^{2}\left(A^{Z}(X), Y\right)=\langle B(X, Y), Z\rangle \text {. }
$$

Proposição 1.2.2. O operador $A^{Z}$ definido acima tem caráter tensorial em $X$ e $Z$ e é auto-adjunto, isto é, $d s^{2}\left(A^{Z}(X), Y\right)=d s^{2}\left(X, A^{Z}(Y)\right)$.

Demonstração: $A^{Z}$ tem caráter tensorial em $X$ devido ao caráter tensorial de $B$. O mesmo ocorre para $Z$ quanto ao tensor métrico $\langle$,$\rangle em \mathbb{R}^{3} . A^{Z}$ é auto adjunto pois $d s^{2}\left(A^{Z}(X), Y\right):=\langle B(X, Y), Z\rangle=\langle B(Y, X), Z\rangle=: d s^{2}\left(A^{Z}(Y), X\right)=d s^{2}\left(X, A^{Z}(Y)\right)$.

Definição 1.2.3. O operador $A^{Z}: \mathscr{X}(M) \rightarrow \mathscr{X}(M)$ definido acima é chamado tensor de Weingarten de $S$ (ou de $f$ ).

Para a segunda forma fundamental, $B(X, Y)$, podemos definir seus coeficientes num sistema de coordenadas $(U, w=u+i v)$ :

$$
\begin{array}{ll}
b_{11}:=B\left(\partial_{u}, \partial_{u}\right) ; \quad b_{12}:=B\left(\partial_{u}, \partial_{v}\right) ; \\
b_{21}:=B\left(\partial_{v}, \partial_{u}\right) ; \quad b_{22}:=B\left(\partial_{v}, \partial_{v}\right) .
\end{array}
$$

Como $B$ é simétrica $b_{12}=b_{21}$. As funções $b_{i j}, i \leq j, j=1,2$ são os coeficientes da segunda forma fundamental de $S$ relativamente ao sistemas de coordenadas $(U, w)$. Podemos então escrever

$$
B=b_{11} d u^{2}+2 b_{12} d u d v+b_{22} d v^{2}
$$

ao longo de $U$, onde $d u^{2}:=d u \otimes d u, d v^{2}=d v \otimes d v$ e $d u d v=\frac{d u \otimes d v+d v \otimes d u}{2}$.

Como em cada $T_{p} M, p \in M$ o operador de Weingarten é auto-adjunto, existe uma base para $T_{p} M$ na qual sua matriz é diagonal. Os autovalores do operador de Weingarten em $p$ são chamados curvaturas principais, enquanto que os autovetores são chamados vetores principais de $T_{p} M$.

No caso do $\mathbb{R}^{3}$, em que tratamos neste trabalho, a codimensão do espaço tangente é 1 e portanto $B_{p}(X, Y)$ será paralelo ao campo normal unitário de $S \subset \mathbb{R}^{3} \mathrm{em} f(p)$. A aplicação $N: M \rightarrow S^{2}(1)$, que a cada $p \in M$ associa o vetor unitário ortogonal ao 
longo de $S$, é chamada aplicação normal de Gauss e pode ser dada num sistema de coordenadas $(U, w=u+i v)$ de $M$ por

$$
N(u+i v)=\frac{f_{u} \wedge f_{v}}{\left\|f_{u} \wedge f_{v}\right\|},
$$

onde $\wedge$ denota o produto vetorial usual do $\mathbb{R}^{3}$.

Assim, $B_{p}(X, Y)=\mu(p) N_{f(p)}$. Da definição de $B$ devemos ter

$$
\mu(p)=\left\langle\left(\nabla_{X}^{f} Y\right)_{p}, N_{f(p)}\right\rangle .
$$

$\operatorname{Mas}\left(\nabla_{X}^{f} Y\right)_{p}=f_{x y}(p)$, se $x=X(p)$ e $y=Y(p)$ e com isso temos em coordenadas:

$$
b_{11}=\left\langle f_{u u}, N\right\rangle ; \quad b_{12}=\left\langle f_{u v}, N\right\rangle=b_{21} ; \quad b_{22}:=\left\langle f_{v v}, N\right\rangle .
$$

Se $(U, u+i v)$ é um sistema de coordenadas de $M$ então em cada $p \in M$ temos que $A:=A^{N}$ tem como matriz relativamente à base $\left\{\partial_{u}, \partial_{v}\right\}$ em $T_{p} M$

$$
A=\left(\begin{array}{ll}
b_{11} & b_{12} \\
b_{12} & b_{22}
\end{array}\right) \text {. }
$$

Como $\left\langle f_{u}, N\right\rangle \equiv 0$, obtemos $b_{11}=\left\langle f_{u u}, N\right\rangle=-\left\langle f_{u}, N_{u}\right\rangle$ e de modo análogo, $b_{12}=-\left\langle f_{u}, N_{v}\right\rangle=-\left\langle f_{v}, N_{u}\right\rangle$ e $b_{22}=-\left\langle f_{v}, N_{v}\right\rangle$. Portanto, $A=-d N$.

No caso geral, definimos o campo vetorial curvatura média $\vec{H}$ ao longo de $S$ como sendo

$$
\vec{H}=\frac{1}{2} \operatorname{tr}(B) \in \mathscr{X}(f)^{\perp} .
$$

Em uma superfície $S=f(M)$ do $\mathbb{R}^{3}$, como $B$ é paralelo ao campo normal de $S$, temos em um sistema de coordenadas isotérmico $(U, u+i v)$ que

$$
\begin{aligned}
\vec{H} & =\frac{1}{2} \operatorname{tr}(B)=\frac{1}{2} \sum_{i=1}^{2} B\left(e_{i}, e_{i}\right) \\
& =\frac{1}{2}\left\langle\sum_{i=1}^{2} B\left(e_{i}, e_{i}\right), N\right\rangle N \\
& =\frac{1}{2} \sum_{i=1}^{2}\left\langle e_{i}, A\left(e_{i}\right)\right\rangle N \\
& =\frac{1}{2 \lambda^{2}}\left(\left\langle\partial_{u}, A\left(\partial_{u}\right)\right\rangle+\left\langle\partial_{v}, A\left(\partial_{v}\right)\right\rangle\right) N \\
& =\frac{1}{2 \lambda^{2}}\left(b_{11}+b_{22}\right) N
\end{aligned}
$$

onde $e_{1}:=\frac{1}{\lambda} \partial_{u}$ e $e_{2}:=\frac{1}{\lambda} \partial_{v}$ 
Definição 1.2.4. Seja $S=f(M)$ uma superfície em $\mathbb{R}^{3}$. Definimos

$$
\begin{aligned}
K & :=\operatorname{det}(A), \\
H & :=\frac{1}{2} \operatorname{tr}(A),
\end{aligned}
$$

como sendo, respectivamente, as curvaturas Gaussiana e média de $S$.

Observe que, pela definição acima, $K$ depende da geometria extrínseca de $S=$ $f(M)$. Porém Gauss provou que este objeto é intrínseco, dependendo somente da métrica em $M$. Estes objetos estão bem definidos pois tanto o determinante quanto o traço da matriz de um operador linear independem da base escolhida.

Agora podemos observar uma importante relação entre o operador de LaplaceBeltrami de uma superfície e seu vetor curvatura média.

Definição 1.2.5. O operador de Lapace-Beltrami de $M$, que denotamos $\Delta_{M}$ é definido como

$$
\Delta_{M}(\cdot):=\operatorname{div}\left(\nabla_{M}(\cdot)\right)
$$

onde div representa o divergente e $\nabla_{M}$ o gradiente em $M$ relativos a $d s^{2}$.

Proposição 1.2.6. Se $\Delta$ é o operador de Laplace-Beltrami definido em coordenadas $(U, w=u+i v)$ no plano por $\Delta=\frac{\partial^{2}}{\partial u^{2}}+\frac{\partial^{2}}{\partial v^{2}}$ e $\Delta_{M}$ é o operador de Laplace-Beltrami num sistema de coordenadas isotérmico de $M$ então

$$
\Delta_{M}(\cdot)=\frac{1}{\lambda^{2}} \Delta(\cdot) \circ w^{-1}, \lambda=\left\langle f_{u}, f_{u}\right\rangle^{\frac{1}{2}}=\left\langle f_{v}, f_{v}\right\rangle^{\frac{1}{2}} .
$$

Demonstração: Ver [4].

Teorema 1.2.7. Sejam $M$ uma superfície de Riemann, $f: M \hookrightarrow \mathbb{R}^{3}$ uma imersão $C^{\infty}$ com coordenadas $f_{i}, i=1 \ldots 3$ e $S=f(M)$ a superfície do $\mathbb{R}^{3}$ com parâmetros isotérmicos dada por $f$ e $M$. Se $\vec{H}$ é o vetor curvatura média de $S$ (ou de f) então

$$
\Delta_{M} f:=\left(\Delta_{M} f_{1}, \Delta_{M} f_{2}, \Delta_{M} f_{3}\right)=2 \vec{H}
$$

Demonstração: $\operatorname{Seja}(U, w=u+i v) \in \mathcal{H}(M)$ então $\left\langle f_{u}, f_{u}\right\rangle=\left\langle f_{v}, f_{v}\right\rangle=: \lambda^{2}$ e $\left\langle f_{u}, f_{v}\right\rangle \equiv 0$. Além disso, $\left\langle f_{u}, N\right\rangle \equiv 0$ e $\left\langle f_{v}, N\right\rangle \equiv 0$. Donde, derivando em $u$ e $v$, obtemos

$$
\begin{array}{ll}
\left\langle f_{u u}, N\right\rangle=-\left\langle f_{u}, N_{u}\right\rangle, & \left\langle f_{v v}, N\right\rangle=-\left\langle f_{v}, N_{v}\right\rangle, \\
\left\langle f_{u u}, f_{u}\right\rangle=\left\langle f_{u v}, f_{v}\right\rangle, & \left\langle f_{v v}, f_{v}\right\rangle=\left\langle f_{u v}, f_{u}\right\rangle, \\
\left\langle f_{u u}, f_{v}\right\rangle=-\left\langle f_{u}, f_{v u}\right\rangle, & \left\langle f_{u v}, f_{v}\right\rangle=-\left\langle f_{u}, f_{v v}\right\rangle .
\end{array}
$$


Lembrando que $\Delta_{M} f=\frac{1}{\lambda^{2}}\left(f_{u u}+f_{v v}\right)$ temos

$$
\begin{aligned}
\left\langle\Delta_{M} f, N\right\rangle & =\frac{1}{\lambda^{2}}\left(\left\langle f_{u u}, N\right\rangle+\left\langle f_{v v}, N\right\rangle\right) \\
& =\frac{1}{\lambda^{2}}\left(-\left\langle f_{u}, N_{u}\right\rangle-\left\langle f_{v}, N_{v}\right\rangle\right) \\
& =\frac{1}{\lambda^{2}}\left(b_{11}+b_{22}\right) \\
& =2\langle\vec{H}, N\rangle .
\end{aligned}
$$

Mas, como $\vec{H} \in \mathscr{X}(f)^{\perp}$ e $|N|=1$ temos que

$$
\Delta_{M} f=2 \vec{H}=2 H N
$$

para $H:=\frac{1}{2 \lambda^{2}}\left(b_{11}+b_{22}\right)$

Observação: Da fórmula acima, tiramos a relação entre a curvatura média e o operador de Laplace-Beltrami em coordenadas $(U, w=u+i v)$ :

$$
\Delta f \circ w^{-1}=4 f_{w \bar{w}}=2 \lambda^{2}\left(\vec{H} \circ w^{-1}\right) \Rightarrow f_{w \bar{w}}=\frac{\lambda^{2}}{2} \vec{H} \circ w^{-1} .
$$

Se $V$ é um espaço vetorial sobre $\mathbb{R}$ indiquemos o seu complexificado por $V^{\mathbb{C}}:=$ $\{u+i v: u, v \in V\}$. Se $V$ possui um produto interno $\langle$,$\rangle , podemos estendê-lo a V^{\mathbb{C}}$ do seguinte modo

$$
\langle u+i v, x+i y\rangle^{\mathbb{C}}:=\langle u, x\rangle-\langle v, y\rangle+i(\langle u, y\rangle+\langle v, x\rangle) .
$$

Note que $\operatorname{dim}_{\mathbb{R}} V=\operatorname{dim}_{\mathbb{C}} V^{\mathbb{C}}$.

Voltando para a segunda forma fundamental de $S=f(M)$, podemos considerar $T_{p} M^{C}$ e $B^{C}$.

Definição 1.2.8. Se $u, v, x, y \in T_{p} M$,

$$
B_{p}^{\mathbb{C}}(u+i v, x+i y):=B_{p}(u, x)-B_{p}(v, y)+i\left(B_{p}(u, y)+B(v, x)\right) .
$$

$B^{\mathbb{C}}$ é então a segunda forma fundamental complexificada de $S$.

Proposição 1.2.9. Sejam $S=f(M)$ uma superfície conforme do $\mathbb{R}^{3}$ e $(U, w=$ $u+i v) \in \mathcal{H}(M)$ então

$$
B^{\mathbb{C}}:=B^{\mathbb{C}}\left(\partial_{w}, \partial_{w}\right) d w^{2}+2 B^{\mathbb{C}}\left(\partial_{w}, \partial_{\bar{w}}\right) d w d \bar{w}+B^{\mathbb{C}}\left(\partial_{\bar{w}}, \partial_{\bar{w}}\right) d \bar{w}^{2}
$$

onde $\partial_{w}=\frac{1}{2}\left(\partial_{u}-i \partial_{v}\right), \partial_{w}=\frac{1}{2}\left(\partial_{u}+i \partial_{v}\right), d w=d u+i d v, d \bar{w}=d u-i d v, d w^{2}=d w d w$, $d w d \bar{w}=\frac{d w \otimes d \bar{w}+d \bar{w} \otimes d w}{2} e d \bar{w}^{2}=d \bar{w} d \bar{w}$. 
Demonstração: Conseqüência direta da definição acima e da bilinearidade de $B$

Vamos omitir $\mathbb{C}$ em $B^{\mathbb{C}}$ sempre que não houver ambigüidade. Da definição acima temos que

$$
B=\left[\left\langle f_{w w}, N\right\rangle^{\mathbb{C}} d w^{2}+2\left\langle f_{w \bar{w}}, N\right\rangle^{\mathbb{C}} d w d \bar{w}+\left\langle f_{\overline{w w}}, N\right\rangle^{\mathbb{C}} d \bar{w}^{2}\right] N
$$

Definição 1.2.10. Seja $B$ a segunda forma fundamental complexificada de $S=$ $f(M)$; então definimos

$$
\begin{aligned}
B^{2,0} & :=\left\langle f_{w w}, N\right\rangle^{\mathbb{C}} d w^{2} \\
B^{1,1} & :=2\left\langle f_{w \bar{w}}, N\right\rangle^{\mathbb{C}} d w d \bar{w} \\
B^{0,2} & :=\left\langle f_{\overline{w w}}, N\right\rangle^{\mathbb{C}} d \bar{w}^{2} .
\end{aligned}
$$

Então $B^{\mathbb{C}}=B^{2,0}+B^{1,1}+B^{0,2}$.

Proposição 1.2.11. Sejam $S=f(M)$ superfície conforme com $B$ e $\vec{H}$ sendo a segunda forma fundamental e o vetor curvatura média, respectivamente, e $(U, w=$ $u+i v) \in \mathcal{H}(M)$. Então para $\lambda^{2}=\left\langle f_{u}, f_{u}\right\rangle=\left\langle f_{v}, f_{v}\right\rangle$ temos

$$
\begin{aligned}
& b_{11}=2 \Re\left\langle f_{w w}, N\right\rangle^{\mathbb{C}}-\lambda^{2}\langle\vec{H}, N\rangle, \\
& b_{22}=-2 \Re\left\langle f_{w w}, N\right\rangle^{\mathbb{C}}-\lambda^{2}\langle\vec{H}, N\rangle, \\
& b_{12}=-2 \Im\left\langle f_{w w}, N\right\rangle^{\mathbb{C}} .
\end{aligned}
$$

Demonstração: Basta usar a expressão para $B^{\mathbb{C}}$ em coordenadas e a equação (1.7).

\subsection{Superfícies de Riemann}

Como vimos no teorema 1.1.5, se $S=f(M)$ é uma superfície conforme em $\mathbb{R}^{3}$ então podemos induzir uma estrutura holomorfa sobre $M$. Nesta seção vamos enunciar alguns resultados sobre superfícies de Riemann que serão utilizados ao longo do texto.

Definição 1.3.1. Uma superfície de Riemann $M$ é uma variedade bidimensional, conexa, de Hausdorff (isto é, dados dois pontos distintos $p$ e $q \in M$ existem abertos disjuntos $U$ e $V$ contendo $p$ e $q$ respectivamente) com um atlas máximo $\mathcal{A}$ cujas mudanças de coordenadas são funções analíticas complexas. 
Como exemplos de superfícies de Riemann temos o plano complexo $\mathbb{C}$ e qualquer domínio nele, isto é, abertos conexos de $\mathbb{C}$, sendo as estruturas complexas dadas pelos atlas máximos que contêm a identidade.

A esfera unitária $\hat{\mathbb{C}}:=\mathbb{C} \cup\{\infty\}$ é outro exemplo de grande importância para a teoria. Para dar estrutura complexa a esse conjunto, definimos a seguinte topologia sobre ele: os abertos de $\hat{\mathbb{C}}$ são os abertos de $\mathbb{C}$ mais os conjuntos da forma $V \cup\{\infty\}$, onde $\mathbb{C} \backslash V$ é compacto. Com essa topologia $\hat{\mathbb{C}}$ é de Hausdorff e homeomorfo a $S^{2}(1)$. Definindo $U_{1}=\hat{\mathbb{C}} \backslash\{\infty\}, \varphi_{1}: U_{1} \rightarrow \mathbb{C}$ dada por $\varphi_{1}(z)=z$ e $U_{2}=\hat{\mathbb{C}} \backslash\{0\}, \varphi_{2}: U_{2} \rightarrow \mathbb{C}$ dada por $\varphi_{2}(z)=\frac{1}{z}$ temos que $\hat{\mathbb{C}}$ é conexo, pois para $i=1,2, \varphi_{i}$ é homeomorfismo, $U_{1} \cap U_{2} \neq \emptyset$ e cada $U_{i}$ é conexo. Vamos mostrar agora que $\varphi_{1}$ e $\varphi_{2}$ são compatíveis na interseção de seus domínios. Observemos que $U_{1} \cap U_{2}=\mathbb{C} \backslash\{0\}=: \mathbb{C}_{*}$ e que $\varphi_{i}\left(U_{1} \cap U_{2}\right)=\mathbb{C}_{*}$. Das expressões em coordenadas de $\varphi_{i}(z)$ temos

$$
\left.\varphi_{2} \circ \varphi_{1}^{-1}\right|_{U_{1} \cap U_{2}}=\left.\varphi_{1} \circ \varphi_{2}^{-1}\right|_{U_{1} \cap U_{2}}=\frac{1}{z}
$$

que é biholomorfismo de $U_{1} \cap U_{2}$. Para dar uma estrutura complexa para $\hat{\mathbb{C}}$ basta então considerar o atlas máximo que contenha $\left(U, \varphi_{1}\right)$ e $\left(U, \varphi_{2}\right)$.

Definição 1.3.2. Seja $M$ uma superfície de Riemann e $X \subset M$ um aberto. Uma função contínua $f: X \rightarrow \mathbb{C}$ é holomorfa (respectivamente meromorfa) se para todo sistema de coordenadas $(U, z)$ de $M$ tal que $U \cap X \neq \emptyset$ temos que $f \circ z^{-1}: z(U \cap X) \rightarrow$ $\mathbb{C}$ é holomorfa (respectivamente meromorfa). Indicaremos por $\mathcal{O}(X)$ (respectivamente $\mathcal{M}(X))$ o conjunto das funções holomorfas (respectivamente meromorfas) de $X \subset M$ em $\mathbb{C}$.

Definição 1.3.3. Sejam $M$ uma superfície de Riemann e $f: M \rightarrow \mathbb{C}$ uma função holomorfa não constante. Um ponto $p \in M$ é um ponto de ramificação se não existe nenhuma vizinhança $V$ de $p$ tal que $\left.f\right|_{V}$ é injetora. Se a função $f$ não possui pontos de ramificação dizemos que ela é uma função holomorfa não ramificada.

Teorema 1.3.4. Seja $M$ uma superfície de Riemann. Uma função $f: M \rightarrow \mathbb{C}$ não tem pontos de ramificação se e somente se f é homeomorfismo local.

Demonstração: Ver [8].

Como exemplos de aplicações com pontos de ramificação temos as funções de $f_{k}: \mathbb{C} \rightarrow \mathbb{C}$ dadas por $f_{k}(z)=z^{k}, k \geq 2$. Para elas $0 \in \mathbb{C}$ é ponto de ramificação pois para cada $\rho>0, V:=\{z \in \mathbb{C}:|z|<\rho$ e $b \in V \backslash\{0\}\}$, existem $a_{1}, \ldots, a_{k} \in\{z \in \mathbb{C}:$ $\left.|z|<\rho^{1 / n}\right\}$ distintos tais que $f_{k}\left(a_{j}\right)=b, 1 \leq j \leq k$.

Um função holomorfa não ramificada é exp $: \mathbb{C} \rightarrow \mathbb{C}_{*}$ pois ela é injetora em cada aberto $V \subset \mathbb{C}$ que não contenha dois pontos cuja diferença seja um múltiplo inteiro de $2 \pi i$. 
Podemos descrever localmente o comportamento de funções holomorfas em superfícies de Riemann. Sejam $M$ uma superfície de Riemann e $(U, z)$ uma coordenada local para $M$ e $V$ um aberto de $\mathbb{C}$ com $p \in U$. Uma vez que as tranlações são biholomorfismos de $\mathbb{C}$, se $f \in \mathcal{O}(M)$ e $p \in M$ podemos supor $0 \in V$ com $f(p)=0$ e $z(p)=0$. Então $f$ tem a seguinte expressão em relação à coordenada $z$ :

$$
f(z)=z^{k}\left(c_{0}+c_{1} z+c_{2} z^{2}+\ldots\right)=z^{k} g(z)
$$

onde $g(z) \in \mathcal{O}(z(U)), g(0) \neq 0$ e sua expansão em série de Taylor com relação à coordenada $z$ é $g(z)=\sum_{j=1}^{\infty} c_{j} z^{j}$. Como $g(0) \neq 0$ existe uma vizinhança simplesmente conexa de $0 \in z(U)$ onde $g(z)$ não se anula e uma função $h$ nessa vizinhança tal que $g(z)=(h(z))^{n}$. Considerando a função $\xi(z)=z h(z)$ temos que $\xi \circ z$ é coordenada local de $M$ em torno de $p$ e com relação a essas coordenadas temos

$$
f(\xi)=\xi^{k}
$$

Podemos interpretar o número $k$ do seguinte modo: para cada vizinhança $U$ de $p$ existem vizinhanças $V \subset U$ de $p$ e $W$ de $q=f(p)$ tais que $f^{-1}(y) \cap V$ consiste exatamente de $k$ pontos para todo $y \in W$ se $y \neq q$.

Definição 1.3.5. O número $k$ acima é chamado de multiplicidade de $f$ no ponto $p$, denotado por $m(f, p)$, e $k-1$ é o índice de ramificação ou ordem de ramificação de $f$ no ponto $p$, denotado por $i(f, p)$. Quando o valor $q$ tiver multiplicidade $k$ escreveremos $q^{(k)}$

Definição 1.3.6. Seja $f \in \mathcal{M}(M)$ e $p \in M$. O ponto $p$ é um zero de multiplicidade $k$ de $f$ se $f(p)=0$ e além disso para uma coordenada $(z, U)$ com $z(p)=a$ temos

$$
f(z)=\sum_{j=k}^{\infty} c_{j}(z-a)^{j}=c_{k}(z-a)^{k}+c_{k+1}(z-a)^{k+1}+\ldots
$$

Definição 1.3.7. Sejam $M$ uma superfície de Riemann e $f \in \mathcal{M}(M)$. Os pontos $p \in M$ tais que $\lim _{z \rightarrow p}|f(z)|=\infty$ são chamados pólos de $f$. Um pólo tem multiplicidade $k$ se a série de Laurent de $f$ com relação à coordenada $z$ em torno de $z(p)=a$ tem a forma:

$$
f(z)=\sum_{j=-k}^{\infty} c_{j}(z-a)^{j}
$$

Teorema 1.3.8 (Teorema das singularidades removiveis de Riemann). Sejam $M$ uma superfície de Riemann, $U \subset M$ aberto e $a \in U$. Suponha que $f \in \mathcal{O}(U \backslash\{a\})$ é limitada numa vizinhança de a. Então $f$ pode ser estendida unicamente a uma função $\tilde{f} \in \mathcal{O}(U)$. 
Demonstração: Ver [8].

Teorema 1.3.9. Sejam $M$ uma superficie de Riemann e $f, g \in \mathcal{O}(M)$. Se $f$ e $g$ coincidem num conjunto com ponto de acumulação de $M$ então $f \equiv g$.

Demonstração: Ver [8].

Definição 1.3.10. Sejam $M$ e $N$ superfícies de Riemann, $X \subset M$ e $Y \subset N$ abertos. Uma função contínua $f: X \rightarrow Y$ é holomorfa (respec. meromorfa) se para toda carta $(z, U)$ de $M$ e toda carta $(w, V)$ de $N$ tais que $U \cap X \neq \emptyset$ e $V \cap Y \neq \emptyset$ temos que $w \circ f \circ z^{-1}: z(U \cap X) \rightarrow w(V \cap Y)$ é holomorfa (respec. meromorfa).

A seguir listamos alguns resultados que são consequências dos resultados acima, cujas demonstrações são simples e podem ser encontradas em [8].

Corolário 1.3.11. Sejam $M, N$ superfícies de Riemann e $f: M \rightarrow N$ uma função holomorfa não constante. Então $f$ é aberta, ou seja a imagem por $f$ de qualquer aberto de $M$ é aberto em $N$.

Corolário 1.3.12. Sejam $M, N$ superficies de Riemann e $f: M \rightarrow N$ uma função holomorfa injetora. Então f é um biholomorfismo.

Corolário 1.3.13 (Princípio do máximo). Sejam $M$ uma superfície de Riemann e $f: M \rightarrow \mathbb{C}$ uma função holomorfa não constante. Então $|f|$ não atinge máximo em $M$.

Teorema 1.3.14. Sejam $M$ e $N$ superficies de Riemann, com $M$ compacta e $f$ : $M \rightarrow N$ uma função holomorfa não constante. Então $N$ é compacta e f é sobrejetora.

Corolário 1.3.15. Toda função holomorfa de uma superfície de Riemann compacta em $\mathbb{C}$ é constante.

Podemos com esses resultados classificar todas as funções meromorfas da esfera de Riemann $\hat{\mathbb{C}}$ em $\mathbb{C}$, ou equivalentemente as funções holomorfas de $\hat{\mathbb{C}}$ em $\hat{\mathbb{C}}$.

Teorema 1.3.16. Seja $f \in \mathcal{M}(\hat{\mathbb{C}})$. Então $f$ é racional.

Demonstração: A função $f$ tem um número finito de pólos, pois em caso contrário, como $\hat{\mathbb{C}}$ é compacto essa sequência de pólos teria um subsequência convergente de pólos, existindo então um ponto de acumulação o que implica $f \equiv \infty$. Podemos assumir que $f(\infty) \neq \infty$, pois caso contrário considere uma aplicação $\varphi$ de Möebius que leva $\infty$ em 0 . Compondo $f \operatorname{com} \varphi$ temos que $\infty$ não é mais pólo de $f$ e claramente esta composta também é meromorfa (todo biholomorfismo de $\hat{\mathbb{C}}$ é uma aplicação de 
Möebius). Sejam então $p_{1}, p_{2}, \ldots, p_{n} \in \mathbb{C}$ os pólos de $f$ e $h_{\nu}(z)=\sum_{j=-k_{\nu}}^{-1} c_{\nu_{j}}\left(z-a_{\nu}\right)^{j} \mathrm{a}$ parte principal da série de Laurent de $f$ em torno do pólo $p_{\nu}, \nu=1, \ldots, n$. Então a função

$$
g:=f-\sum_{\nu=1}^{n} h_{\nu}
$$

é holomorfa em $\hat{\mathbb{C}}$ e portanto constante pelo corolário 1.3.15. Logo $f$ é racional, pois

$$
f=c t e+\sum_{\nu=1}^{n} h_{\nu}
$$

Teorema 1.3.17 (Teorema de Liouville). Toda função holomorfa $f: \mathbb{C} \rightarrow \mathbb{C}$ limitada é constante.

Demonstração: Pelo teorema $1.3 .8 f$ pode ser estendida analiticamente a uma função holomorfa $\tilde{f}: \hat{\mathbb{C}} \rightarrow \mathbb{C}$ que é constante pelo corolário 1.3.15. Donde $f$ também é constante.

Teorema 1.3.18. Sejam $M$ uma superfície de Riemann e $f, g$ funções meromorfas sobre $M$. Se $f$ e g têm os mesmos zeros e pólos então $g=c f, c \in \mathbb{C}$ constante.

Demonstração: Uma versão para funções no plano complexo pode ser encontrada em [3]. Para superfícies de Riemman basta importar esse resultado através de suas estruturas holomorfas.

Definição 1.3.19. Uma forma diferencial holomorfa (respc. meromorfa) $\eta$ sobre uma superfície de Riemann $M$ é uma lei que associa a cada sistema de coordenadas $\left(U_{\alpha}, z_{\alpha}\right)$ de sua estrutura holomorfa uma função holomorfa (respec. meromorfa) $f_{\alpha}$ : $z_{\alpha}\left(U_{\alpha}\right) \rightarrow \mathbb{C}$ com a propriedade

$$
f_{\alpha}\left(z_{\alpha}\right)=\varphi_{\alpha \beta}^{\prime}\left(z_{\alpha}\right) f_{\beta}\left(\varphi_{\alpha \beta}\left(z_{\alpha}\right)\right), \text { onde }
$$

$\left(U_{\alpha}, z_{\alpha}\right)$ e $\left(U_{\beta}, z_{\beta}\right)$ são sistemas de coordenadas de $M$ tais que $U_{\alpha} \cap U_{\beta} \neq \emptyset$ e $\varphi_{\alpha \beta}:=$ $z_{\beta} \circ z_{\alpha}^{-1}$.

Para as formas diferenciais temos um resultado análogo ao teorema 1.3.18:

Teorema 1.3.20. Sejam $M$ uma superficie de Riemann e $\eta, \omega$ diferenciais meromorfas sobre $M$. Se $\eta$ e $\omega$ têm os mesmos zeros e pólos então $\omega=c \eta, c \in \mathbb{C}$ constante. 
Demonstração: Localmente podemos escrever $\eta=f_{\alpha} d z_{\alpha}$. Aplicando o teorema 1.3 .18 a $f_{\alpha}$ e usando a conexão por caminhos de $M$ obtemos o resultado.

Localmente representamos a diferencial $\eta$ acima por $\eta_{\alpha}=f_{\alpha} d z_{\alpha}$. Usamos as diferenciais para fazer integração ao longo de caminhos numa superfície de Riemann $M$. Se $\gamma=\gamma(t):[a, b] \rightarrow M$ é um caminho de classe $C^{1}$ por partes sobre $M$ e $U_{1}, U_{2}, \ldots, U_{k}$ são vizinhanças coordenadas de $M \operatorname{com}\{\gamma\} \subset \bigcup_{j=1}^{k} U_{j}$ e $\eta_{j} d z_{j}$ são as representações locais de uma diferencial meromorfa $\eta$ sobre $M$ definimos

$$
\int_{\gamma} \eta=\sum_{j=1}^{k} \int_{\gamma_{j}} \eta_{j}=\sum_{j=1}^{k} \int f_{j} d z_{j}
$$

onde $\gamma_{j}=\left.\gamma\right|_{\left[a_{j-1}, a_{j}\right]}, a_{0}=a, a_{k}=b$. A integral acima está bem definida, devido à condição de compatibilidade na definição de diferenciais e com isso a integração delas resume-se localmente à integração usual de funções complexas no plano.

Definição 1.3.21. Duas curvas $\gamma=\gamma(t)$ e $\tilde{\gamma}=\tilde{\gamma}(t)$ numa superfície de Riemann $M$ são homotópicas se elas têm os mesmos extremos, isto é, $\gamma(0)=\tilde{\gamma}(0)=$ : $a$ e $\gamma(1)=\tilde{\gamma}(1)=: b$, e se existe uma aplicação contínua $A=A(s, t):[0,1] \times[0,1] \rightarrow M$ que satisfaz $A(0, t)=\gamma(t), A(1, t)=\tilde{\gamma}(t), A(s, 0)=a$ e $A(s, 1)=b$ para todo $s$ e $t$ em $[0,1] \times[0,1]$

Teorema 1.3.22. Seja $M$ uma superfície de Riemann e $\eta$ uma forma diferenciável sobre $M$ e $\gamma$ e $\tilde{\gamma}$ caminhos sobre $M$. Então se $\gamma$ é homotópica a $\tilde{\gamma}$ temos que

$$
\int_{\gamma} \eta=\int_{\tilde{\gamma}} \eta
$$

Demonstração: Segue do teorema de Cauchy para funções complexas de $\mathbb{C}$ que pode ser encontrado em [3].

Definição 1.3.23. Seja $\eta$ uma diferencial sobre uma superfície de Riemann. Definimos o grau de $\eta$, deg $(\eta)$, como a diferença entre o número de zeros e pólos de $\eta$, contando suas multiplicidades.

Toda superfície topológica orientável e compacta é homeomorfa a uma esfera com $g \geq 0$ alças. A teoria de superfícies topológicas pode ser encontrada em [11]. A adição de alças a uma superfície de Riemann está feita em [15].

Definição 1.3.24. O gênero de uma superfície topológica orientável e compacta é definido como o inteiro $g$ acima. 
Teorema 1.3.25. Seja $M$ uma superfície topológica orientável e compacta de gênero g.A característica de Euler de $M$ é

$$
\chi(M)=2(1-g) .
$$

Demonstração: Ver [11]

Teorema 1.3.26. Nas condições da definição 1.3.23, se $M$ é compacta então,

$$
\operatorname{deg}(\eta)=-\chi(M)
$$

Demonstração: Ver [2].

Vamos definir agora o resíduo de uma funcão meromorfa num pólo isolado, o que será muito útil para a resolucão de alguns problemas de períodos em superfícies mínimas. Para isso observemos que se $f: \mathbb{C} \rightarrow \mathbb{C}$ é uma função meromorfa e $a \in \mathbb{C}$ é um pólo de $f$ então temos sua expansão em série de Laurent

$$
f(z)=\sum_{j=-k}^{\infty} c_{j}(z-a)^{j}
$$

com

$$
c_{j}=\frac{1}{2 \pi i} \int_{\gamma} \frac{f(\zeta)}{(\zeta-a)^{j+1}} d \zeta, k \in \mathbb{N}
$$

onde $\gamma$ é uma curva simples fechada qualquer, percorrida no sentido positivo de orientação, tal que o único pólo em seu interior é $z=a$.

Definição 1.3.27. Sejam $f: \mathbb{C} \rightarrow \mathbb{C}$ uma função meromorfa e $a \in \mathbb{C}$ um pólo de $f$. O resíduo de $f$ em $a$ é o coeficiente $c_{-1}$ na série acima e será indicado por res $(f, a)$ ou $\operatorname{res}_{a}(f)$.

Se $a$ é um pólo simples de $f$ então numa vizinhança de a temos a expansão $f(z)=c_{-1}(z-a)^{-1}+c_{0}+c_{1}(z-a)+\ldots$ Se multiplicamos os dois lados por $(z-a)$ e tomamos o limite $z \rightarrow a$ temos

$$
c_{-1}=\lim _{z \rightarrow a}(z-a) f(z)
$$

Se $a$ é um pólo de ordem $k$ de $f$ então sua expansão em série de Laurent fica $f(z)=c_{-k}(z-a)^{-k}+\ldots+c_{-1}(z-a)^{-1}+c_{0}+c_{1}(z-a)+\ldots$ Multiplicando os dois lados por $(z-a)^{k}$, derivando o resultado $k-1$ vezes e então passando o limite $z \rightarrow a$ obtemos

$$
\operatorname{res}(f, a)=c_{-1}=\frac{1}{(k-1) !}\left(\lim _{z \rightarrow a} \frac{d^{k-1}}{d z^{k-1}}\left[(z-a)^{k} f(z)\right]\right)
$$


Teorema 1.3.28 (Teorema do Resíduo). Sejam $f \in \mathcal{M}(\mathbb{C}), a_{k} \in \mathbb{C}, k=1, \ldots, n$ os pólos de $f$ e $\gamma$ uma curva simples fechada orientada positivamente no plano complexo contendo todos $a_{k}$. Então

$$
\int_{\gamma} f(z) d z=2 \pi i \sum_{j=1}^{n} \operatorname{res}\left(f, a_{j}\right) .
$$

Demonstração: Segue do teorema dos resíduos para funções meromorfas complexas.

A seguir listamos alguns resultados sobre a classificação de superfícies de Riemann.

Definição 1.3.29. Duas superfícies de Riemann são conformemente equivalentes se existe um biholomorfismo $f: M \rightarrow N$.

Podemos caracterizar, a menos de biholomorfismos, todas as superfícies de Riemann simplesmente conexas.

Teorema 1.3.30. Toda superfície de Riemann compacta simplesmente conexa é conformemente equivalente à esfera de Riemann $\hat{\mathbb{C}}$.

Mais geralmente temos o resultado a seguir proposto por Riemann em 1851 e generalizado e completamente provado por Henry Poincaré e Paul Koebe em 1907.

Teorema 1.3.31 (Teorema de Koebe). Seja $M$ uma superfície de Riemann simplesmente conexa. Então $M$ é conformemente equivalente à esfera de Riemann $\hat{\mathbb{C}}$, ao plano complexo ou ao disco unitário $D:=\{z \in \mathbb{C}:|z|<1\}$.

Demonstração: Ver [8].

A recíproca para este teorema também é verdadeira, pois $\hat{\mathbb{C}}, \mathbb{C}$ e $D$ são simplesmente conexos e, portanto se uma superfície de Riemann é biholomorfa a qualquer um deles então ela é simplesmente conexa.

Existem superfícies de Riemann compactas que não são simplesmente conexas, como por exemplo o toro. Para um estudo mais profundo dessas superfícies com gênero maior precisamos de alguns resultados de aplicações de recobrimento e funções algébricas, que serão vistos agora.

Proposição 1.3.32 (Fórmula de Euler-Poincaré). Sejam $M$ uma superfície de Riemann e $\chi(M)$ sua característica de Euler. Se $\rho: M \rightarrow M$ tem $k$ pontos fixos e é tal que $\rho^{n}=i d$ então

$$
\chi(M / \rho)=\frac{\chi(M)}{n}+k \frac{n-1}{n} .
$$

Demonstração: Ver [6]. 
Definição 1.3.33. Suponha que $X$ e $Y$ são espaços topológicos de Hausdorff e $\pi$ : $Y \rightarrow X$ é uma função contínua. Para cada $x \in X$ o conjunto $\pi^{-1}(x)$ é chamado de fibra de $\pi$ sobre $x$. Se $y \in \pi^{-1}(x)$ dizemos que $y$ está sobre $x$.

Definição 1.3.34. Se $\pi_{1}: Y \rightarrow X$ e $\pi_{2}: Z \rightarrow X$ são aplicações contínuas entre espaços de Hausdorff então $f: Y \rightarrow Z$ preserva fibras se $\pi_{1}=\pi_{2} \circ f$, isto é, se $y \in Y$ está sobre $x$ então $f(y) \in Z$ também está sobre $x$.

Definição 1.3.35. Uma aplicação entre dois espaços topológicos $\pi: Y \rightarrow X$ é discreta se a fibra de cada $x \in X$ é um conjunto discreto em $Y$.

Teorema 1.3.36. Suponha que $M$ e $N$ são superfícies de Riemann e $\pi: M \rightarrow N$ é uma função holomorfa não constante. Então $\pi$ é aberta e discreta.

Demonstração: Do corolário (1.3.11) temos que $\pi$ é aberta e do teorema (1.3.9) temos que a fibra de qualquer ponto de $N$ deve conter somente pontos isolados, pois caso contrário $\pi$ seria constante.

Freqüentemente encontramos "funções" multivalentes, ou seja um ponto tem diversas imagens por este tipo de função. Isso acontece por exemplo quando temos funções holomorfas $f: M \rightarrow \hat{\mathbb{C}}$ e $\pi: M \rightarrow N$ e desejamos definir uma função de $N$ em $\hat{\mathbb{C}}$ usando $\pi$ e $f$. Para isso observamos que se $q \in N$ então $\pi^{-1}(q)=\left\{p_{j} \in M: j \in J\right\}$, e daí definimos uma função multivalente $\tilde{f}: N \rightarrow \hat{\mathbb{C}}$ dada por $\tilde{f}(x)=\left\{f\left(y_{j}\right): j \in J\right\}$.

O exemplo mais importante dessa situação ocorre quanto tomamos $M=\mathbb{C}, N=$ $\mathbb{C}_{*}, \pi: \mathbb{C} \rightarrow \mathbb{C}_{*}$ dada por $\pi(z)=\exp (z)$ e $f: \mathbb{C} \rightarrow \mathbb{C}$ dada por $\exp \circ f=i d$. Então $f$ corresponde ao logaritmo em $\mathbb{C}_{*}$, pois para cada $q \in \mathbb{C}_{*}$ o conjunto $\exp ^{-1} \circ i d(q)=$ $\exp ^{-1}(q)$ consiste dos infinitos logaritmos de $q$.

Outro exemplo importante é o das raízes n-ésimas. Sabemos que cada valor $z \notin$ $\{0, \infty\}$ de $\hat{\mathbb{C}}$ admite $n$ raízes distintas fazendo então com que a função $\sqrt[n]{z}$ seja multivalente. Veremos mais adiante que podemos definir um espaço topológico com estrutura de superfície de Riemann onde esta função deixa de ser multivalente.

Quando quisermos integrar funções multivalentes devemos escolher o domínio de integração de modo que os pontos de ramificação fiquem fora da região de integração e então consideramos um ramo da função sobre esta região.

Definição 1.3.37. Suponha que $X$ e $Y$ são espaços topológicos. Dizemos que $\pi$ : $Y \rightarrow X$ é uma aplicação de recobrimento se para cada $x \in X$ existir um aberto conexo $U_{x}$ contendo $x$ tal que $\pi^{-1}\left(U_{x}\right)=\bigcup_{y \in \pi^{-1}(x)} V_{y}$ é uma união disjunta dos abertos $V_{y} \subset Y$ e $\left.\pi\right|_{V_{y}}$ é homeomorfismo local para todo $y \in \pi^{-1}(x)$. 
Definição 1.3.38. Suponha que $X, Y$ e $Z$ sejam espaços topológicos e $\pi: Y \rightarrow X$ e $f: Z \rightarrow X$ são funções contínuas. Um levantamento de $f$ com respeito a $\pi$ é uma função contínua $g: Z \rightarrow Y$ tal que $f=\pi \circ g$.

Teorema 1.3.39 (Unicidade do Levantamento). Sejam $X$ e $Y$ espaços de Hausdorff e $\pi: Y \rightarrow X$ um homeomorfismo local. Se $Z$ é um espaço topológico, $f: Z \rightarrow X$ é contínua e $g_{1}$ e $g_{2}$ são dois levantamentos de $f$ com respeito a $\pi$ tais que $g_{1}\left(z_{0}\right)=$ $g_{2}\left(z_{0}\right)$ para algum $z_{0} \in Z$ então, $g_{1} \equiv g_{2}$.

Demonstração: Ver [8].

Teorema 1.3.40. Sejam $X$ e $Y$ espaços de Hausdorff e $\pi: Y \rightarrow X$ um homeomorfismo local. Suponha que $x_{1}, x_{2} \in X$ e $\tilde{x}_{1} \in Y$ tais que $\pi\left(\tilde{x}_{1}\right)=x_{1}$. Seja ainda $A:[0,1] \times[0,1] \rightarrow X$ uma aplicação tal que $A(0, s)=x_{1}$ e $A(1, s)=x_{2}$ para todo $s \in[0,1]$. Se definimos $u_{s}:=A(t, s)$ e temos que cada $u_{s}(t)$ pode ser levantada a uma curva $\tilde{u}_{s}(t)$ com $\tilde{u}_{s}(0)=\tilde{x}_{1}$, então $\tilde{u}_{0}(1)=\tilde{u}_{1}(1)$ e $\tilde{u}_{0}$ e $\tilde{u}_{1}$ são homotópicas.

Demonstração: Ver [8].

Definição 1.3.41. Uma aplicação contínua $\pi: Y \rightarrow X$ tem a propriedade do levantamento de curvas se para toda curva $u:[0,1] \rightarrow X$ e todo ponto $y_{0} \in Y$ com $\pi\left(y_{0}\right)=u(0)$ existe um levantamento $\tilde{u}:[0,1] \rightarrow Y$ tal que $\tilde{u}(0)=y_{0}$.

Teorema 1.3.42. Toda aplicação de recobrimento tem a propriedade do levantamento de curvas.

Demonstração: Ver [8].

Um observação importante é que o levantamento de uma curva fechada, apesar de único quando escolhido o ponto inicial, nem sempre é fechado. Considere $\pi: \mathbb{C}_{*} \rightarrow \mathbb{C}_{*}$ dada por $\pi(z)=z^{2}, x_{0}=y_{0}=1$. Seja $u:[0,1] \rightarrow \mathbb{C}_{*}$ a curva $u(t)=e^{2 \pi i t}$, que é fechada, pois $x_{0}=u(0)=u(1)=1$. O levantamento de $u$ com respeito a $\pi$ é $\tilde{u}(t)=e^{\pi i t}$ cujo ponto inicial $y_{0}=\tilde{u}(0)=1$ e o ponto final é $\tilde{u}(1)=-1$. Porém, do teorema (1.3.40) temos que se a curva for homotopicamente nula seu levantamento também o será. Isso ocorre em particular nos domínios simplesmente conexos.

Teorema 1.3.43. Suponha que $X$ e $Y$ são espaços de Hausdorff com $X$ conexo por caminhos e $\pi: Y \rightarrow X$ uma aplicação de recobrimento. Então para quaisquer pontos $x_{0}, x_{1} \in X$ a cardinalidade de $\pi^{-1}\left(x_{0}\right)$ e $\pi^{-1}\left(x_{1}\right)$ é a mesma.

Demonstração: Ver [8].

Definição 1.3.44. Nas condições do teorema acima, a cardinalidade de $\pi^{-1}(x), x \in X$ é chamada de o número de folhas do recobrimento. 
Esse número de folhas pode ser finito, como no caso das aplicações $z \rightarrow z^{k}, k \in \mathbb{N}$, ou infinito, como no caso da aplicação $z \rightarrow \exp (z)$.

Teorema 1.3.45. Sejam $X$ uma variedade, $Y$ um espaço de Haudorff $e \pi: Y \rightarrow X$ um homeomorfismo local com a propriedade do levantamento de curvas. Então $\pi$ é uma aplicação de recobrimento.

Demonstração: Ver [8].

Definição 1.3.46. Sejam $X$ e $Y$ dois espaços topológicos localmente compactos. Uma função contínua $f: Y \rightarrow X$ é própria se $f^{-1}(K)$ é compacto em $Y$, para todo $K$ compacto de $X$.

Observe que uma aplicação própria é fechada, ou seja, a imagem de qualquer fechado é fechada.

Lema 1.3.47. Sejam $X, Y$ espaços localmente compactos e $\pi: Y \rightarrow X$ uma aplicação discreta própria. Então

(a) Para cada $x \in X, \pi^{-1}(x)$ é finito.

(b) Se $x \in X$ e $V$ é uma vizinhança de $\pi^{-1}(x)$, então existe uma vizinhança $U$ de $x$ tal que $\pi^{-1}(U) \subset V$.

Demonstração: Ver [8].

Teorema 1.3.48. Sejam $X$ e $Y$ espaços localmente compactos $e \pi: Y \rightarrow X$ um homeomorfismo local próprio. Então $\pi$ é aplicação de recobrimento.

Demonstração: Ver [8].

Consideremos agora o conceito de aplicações próprias no contexto de superfícies de Riemann e funções holomorfas.

Se $M$ e $N$ são superfícies de Riemann e $f: M \rightarrow N$ é uma aplicação holomorfa própria não constante, então o conjunto $A$ dos pontos de ramificação de $f$ é fechado e discreto, e como $f$ é própria e portanto fechada, temos que $B:=f(A)$ também é fechado e discreto. Queremos aqui estender o resultado do teorema (1.3.43) para recobrimentos holomorfos ramificados, mas para isso precisamos contar a multiplicidade total de um certo valor $c \in N$ e este valor é dado por

$$
m=\sum_{p \in f^{-1}(c)} m(f, p)
$$

Este valor está bem definido devido à primeira parte do lema (1.3.47), e dos teoremas (1.3.45) (1.3.43)

Assim temos o seguinte teorema: 
Teorema 1.3.49. Sejam $M$ e $N$ superfícies de Riemann e $f: M \rightarrow N$ uma aplicação holomorfa, não constante e própria. Então existe um número natural $n$ tal que todo valor $c \in N$ é assumido exatamente $n$ vezes contando as multiplicidades.

Demonstração: Ver [8].

Portanto uma aplicação holomorfa própria não constante é um recobrimento ramificado com um número definido de folhas e por isso será chamado de recobrimento ramificado de $n$ folhas. Se não houver pontos de ramificação diremos que ela é um recobrimento não ramificado.

Definição 1.3.50. Seja $g: M \rightarrow \hat{\mathbb{C}}$ um recobrimento ramificado de $\hat{\mathbb{C}}$. Então definimos o grau de $g, \operatorname{deg}(g)$, como sendo o número de folhas do recobrimento.

Observe a diferença entre o grau de uma função meromorfa da definição acima e o grau de uma diferencial meromorfa na definição 1.3.23.

Corolário 1.3.51. Sejam $M$ uma superficie de Riemann compacta e $f: M \rightarrow \hat{\mathbb{C}}$ holomorfa. Então $f$ tem o mesmo número de zeros e pólos, contando multiplicidades.

Demonstração: Uma função nas condições acima é própria pois $M$ é compacta.

Definição 1.3.52. Sejam $X$ e $Y$ espaços topológicos e $\pi: Y \rightarrow X$ uma aplicação de recobrimento. Uma transformação de recobrimento é um homeomorfismo $f: Y \rightarrow Y$ que preserva as fibras de $\pi$. Com a operação de composição de funções o conjunto das transformações de recobrimento de $\pi$ é um grupo, indicado por $T(Y / X)$.

Definição 1.3.53. Sejam $X$ e $Y$ espaços de Hausdorff conexos e $\pi: Y \rightarrow X$ uma aplicação de recobrimento. O recobrimento $\pi$ é de Galois se para todos $y_{0}, y_{1} \in Y$ $\operatorname{com} \pi\left(y_{0}\right)=\pi\left(y_{1}\right)$ existe uma tranformação de recobrimento $f$ tal que $f\left(y_{0}\right)=f\left(y_{1}\right)$.

O grupo das tranformações de recobrimento de Galois tem papel importante na construção das superfícies de Riemann associadas a funções algébricas. Ele generaliza o conceito de grupo de Galois para extensões de corpos galoisianas como veremos na próxima seção.

Se $X$ é espaço de Hausdorff conexo então ele admite um recobrimento $\pi: Y \rightarrow X$ com $Y$ simplesmente conexo. Tal recobrimento é chamado recobrimento universal e no caso de superfícies de Riemann é único a menos de equivalência conforme pois se $\pi_{1}: \tilde{M}_{1} \rightarrow M$ e $\pi_{2}: \tilde{M}_{2} \rightarrow M$ são recobrimentos universais de uma superfície de Riemann $M$ então podemos induzir a estrutura complexa de $M$ para $\tilde{M}_{1}$ e $\tilde{M}_{2}$ através de $\pi_{1}$ e $\pi_{2}$ respectivamente. Logo, $\tilde{M}_{1}$ e $\tilde{M}_{2}$ são portanto equivalentemente conformes, considerando o biholomorfismo local definido por $\pi_{2}$ e $\pi_{1}$. 
Se $M$ é conformemente equivalente a $\hat{\mathbb{C}}$ então seu recobrimento universal é $\hat{\mathbb{C}}$, pois $\hat{\mathbb{C}}$ é simplesmente conexo. O teorema a seguir classifica os recobrimentos universais de superfícies de Riemann não conformemente equivalentes a $\hat{\mathbb{C}}$.

Teorema 1.3.54. Sejam $M$ uma superfície de Riemann não conformemente equivalente a $\hat{\mathbb{C}}$ e $\pi: \tilde{M} \rightarrow M$ um recobrimento conforme simplesmente conexo de $M$. Então $\tilde{M}$ é conformemente equivalente ao

(a) plano complexo se $M$ é conformemente equivalente a $\mathbb{C}, \mathbb{C}_{*}$ ou a um toro ou,

(b) ao disco unitário $D=\{z \in \mathbb{C}:|z|<1\}$ nos outros casos.

Demonstração: Ver [2].

Vamos agora definir o gérmen de uma função meromorfa $f(z)=\sum_{\nu=-\infty}^{\infty} c_{\nu}\left(z-z_{0}\right)^{\nu}$ numa vizinhança de um ponto $z_{0} \in \mathbb{C}$. Esta noção nos leva ao conceito de função multivalente como continuação de gérmens de funções meromorfas e à superfície de Riemann de $f$ onde esta função multivalente em $\mathbb{C}$ é univalente e holomorfa. $O$ conceito de gérmen pode ser generalizado para funções meromorfas e para funções holomorfas de $\hat{\mathbb{C}}$ em $\hat{\mathbb{C}}$ de modo análogo, considerando a coordenada $\xi=\frac{1}{z}$, que leva vizinhanças de $\infty$ em vizinhanças de 0 .

Definição 1.3.55. Sejam $z_{0} \in \hat{\mathbb{C}}, r>0$. Um elemento de função meromorfa $f(z)$ é a série de potências

$$
\sum_{\nu=k}^{\infty} c_{\nu}\left(z-z_{0}\right)^{\nu}, k \in \mathbb{Z}, c_{\nu} \in \mathbb{C}
$$

com raio de convergência $r$ e centro $z_{0}$ tal que $f(z)$ seja representada pela série acima em torno de $z_{0}$. O elemento de função será indicado por $\varphi=\left(f, z_{0}, r\right)$. O domínio onde esta série é convergente, isto é, $D=\left\{z \in \hat{\mathbb{C}}: 0<\left|z-z_{0}\right|<r\right\}$ se $z_{0} \neq \infty$ ou $D=\{z \in \hat{\mathbb{C}}:|z|>r\}$, se $z_{0}=\infty$ é chamado domínio do elemento de função $\varphi=\left(f, z_{0}, r\right)$. Indicaremos por $\varphi_{z_{0}}$ um elemento de função centrado em $z_{0}$.

Dois elementos de função $\varphi_{1}$ em $D_{1}$ e $\varphi_{2}$ em $D_{2}$ são continuações analíticas um do outro se $D_{1} \cap D_{2} \neq \emptyset$ e $f_{1}(z)=f_{2}(z)$ em $D_{1} \cap D_{2}$. Isso ocorre por exemplo se $D_{1} \cap D_{2}$ é um aberto de $\hat{\mathbb{C}}$.

Definição 1.3.56. Seja $\gamma=\gamma(t):[0,1] \rightarrow \hat{\mathbb{C}} \operatorname{com} \gamma(0)=p$ e $\gamma(1)=q$. Um gérmen $\psi$ de centro $q$ é a continuação analítica de um gérmen $\varphi$ de centro $p$ ao longo de $\gamma$ se existe uma partição de $[0,1], 0=t_{0}<t_{1}<\ldots<t_{n-1}<t_{n}=1$, abertos $U_{i}, i=1, \ldots, n$ tais que $\gamma\left(\left[t_{i-1}, t_{i}\right]\right) \subset U_{i}$ e uma família de funções holomorfas $f_{i} \in \mathcal{M}\left(U_{i}\right)$ tais que o gérmen $\varphi=\left(f_{1}, p, r_{1}\right)$ e $\psi=\left(f_{n}, q, r_{n}\right)$ e $\left.f_{i}\right|_{V_{i}}=\left.f_{i+1}\right|_{V_{i}}, i=1, \ldots, n-1$, onde $V_{i}$ é a componente conexa de $U_{i} \cap U_{i+1}$ que contém o ponto $\gamma\left(t_{i}\right)$. 
Teorema 1.3.57 (Teorema da Monodromia). Sejam $\gamma_{0}=\gamma_{0}(t), \gamma_{1}=\gamma_{1}(t)$ : $[0,1] \rightarrow \hat{\mathbb{C}}$ curvas homotópicas de extremos $p$ e q. Suponha que o gérmen $\varphi$ admite continuação analítica para cada $\gamma_{s}, 0 \leq s \leq 1$, deformação de $\gamma_{0}$ em $\gamma_{1}$. Então a continuação analítica de $\varphi$ ao longo de $\gamma_{0}$ e $\gamma_{1}$ geram o mesmo gérmem de função em $\gamma_{0}(1)=\gamma_{1}(1)=q$

Demonstração: Ver [8].

Em geral, mesmo quando a continuação analítica de um gérmen é possível ao longo de duas curvas com os mesmos extremos, os gérmens resultantes podem ser diferentes. Se considerarmos todos os gérmens obtidos a partir da continuação analítica de um gérmen obtemos uma função multivalente. O exemplo clássico é o do gérmen $\varphi=$ $(\sqrt{z}, 1,1 / 2)$. Para $\gamma_{1}(t)=1, t \in[0,1]$ a continuação de $\varphi$ ao longo de $\gamma_{1}$ é $\varphi$, enquanto que ao longo de $\gamma_{2}(t)=e^{i t}, 0 \leq t \leq 2 \pi$ a continuação é $(-\sqrt{z},-1,1 / 2)$. Isto está de acordo com a observação após o teorema 1.3.42, pois o levantamento de $\gamma_{2}$ com respeito à função $z \rightarrow z^{2}$ não é uma uma curva fechada. Por outro lado, o gérmen $\varphi=(\sqrt{z}, 1,1 / 2)$ continuado sobre a curva $\gamma=e^{i t}, 0 \leq t \leq 4 \pi$ não sofre alteração. Portanto precisamos de duas voltas em torno da origem para obter o gérmen original, donde temos duas soluções distintas para $w^{2}(z)-z=0$ numa vizinhança de $z=0$. Para a mesma curva no caso de um gérmen do logaritmo nunca voltaremos ao mesmo gérmen, pois todos eles diferem por um múltiplo inteiro de $2 \pi i$.

Se $\Omega$ é um aberto de $\mathbb{C}$, colecionando todas continuações analíticas de um certo gérmen $\varphi_{z_{0}}, z_{0} \in \Omega$ obtemos um conjunto $M$ de fibras com pontos base $z \in \Omega$. A esse conjunto podemos dar uma estrutura de superfície de Riemann, como na proposição abaixo.

Definição 1.3.58. Sejam $z_{0} \in \mathbb{C}$ e $\varphi=\left(f, z_{0}, r\right)$ um elemento de função centrado em $z_{0}$ com $f=\sum_{\nu=k}^{\infty} a_{\nu}\left(z-z_{0}\right)^{\nu}$. Seja ainda $z_{1} \in \mathbb{C}$ tal que $\left|z_{1}-z_{0}\right|=\rho<r$. Então $\tilde{\varphi}=\left(\tilde{f}, z_{1}, \mu\right) \operatorname{com}$

$$
\tilde{f}=\sum_{\nu=k}^{\infty} b_{\nu}\left(z-z_{1}\right)^{\nu}=\sum_{\nu=k}^{\infty} a_{n} u\left[\left(z-z_{1}\right)+\left(z_{1}-z_{0}\right)\right]^{\nu}
$$

é uma continuação imediata de $\varphi$.

Na definição acima podemos verificar com desenvolvimento binomial que a série que representa $\tilde{f}$ é convergente e seu raio de convergência é maior que $r-\rho$.

Proposição 1.3.59. Sejam $M$ o conjunto acima, $\varphi_{z}=(f, z, r)$ e $\epsilon<r$. Então $V_{\epsilon}\left(\varphi_{z}\right)=\left\{\varphi_{\tilde{z}} \in M:|z-\tilde{z}|<\epsilon\right\}$, onde $\varphi_{\tilde{z}}$ é continuação imediata de $\varphi_{z}, z \in \Omega$ formam uma base de abertos para uma topologia em $M$ de Hausdorff e conexa por 
caminhos. Além disso, as funções $\phi: V_{\epsilon}\left(\varphi_{z}\right) \rightarrow \mathbb{C}$ dadas por $\phi\left(\varphi_{\tilde{z}}\right)=\tilde{z}$ fazem parte de um atlas complexo $\mathcal{A}$ que define uma estrutura complexa para $M$.

Demonstração: Diremos que $A \subset M$ é aberto se e só se $A=\bigcup V_{\epsilon}\left(\varphi_{z}\right)$ ou $A=\emptyset$. Claramente os abertos desse tipo cobrem $M$ (isto é, $\bigcup A=M$ ) e $A=\emptyset$ é aberto, faltando verificar apenas que se $A_{1}$ e $A_{2}$ são abertos então $A_{1} \cap A_{2}$ também o é, para mostrar que esta coleção de abertos de fato é uma topologia para $M$. Suponha então que $\varphi_{z} \in A_{1} \cap A_{2}$. Daí existem $\epsilon_{1}, z_{1}$ tais que $\varphi_{z} \in V_{\epsilon_{1}}\left(\varphi_{z_{1}}\right) \subset A_{1}$ e $\epsilon_{2}, z_{2}$ tais que $\varphi_{z} \in V_{\epsilon_{2}}\left(\varphi_{z_{2}}\right) \subset A_{2}$ e ainda podemos supor que $\varphi_{z}$ é continuação imediata de $\varphi_{z_{1}}$ e de $\varphi_{z_{2}}$. Portanto exite $\delta>0$ tal que $V_{\delta}\left(\varphi_{z}\right) \subset \bigcap V_{\epsilon_{j}}\left(\varphi_{z_{k}}\right), k=1,2$ e então $V_{\delta}\left(\varphi_{z}\right) \subset A_{1} \cap A_{2}$. Isto pode ser feito para todo gérmen $\varphi_{z} \in A_{1} \cap A_{2}$. Assim, $A_{1} \cap A_{2}$ é reunião de vizinhanças do tipo $V_{\epsilon}\left(\varphi_{z}\right)$ e portanto aberto.

Vamos mostrar agora que essa topologia em $M$ é de Hausdorff. Para isso suponha que $\varphi_{z_{1}}$ e $\varphi_{z_{2}}$ sejam elementos distintos de $M$. Se $z_{1} \neq z_{2}$ escolhendo $\epsilon<\frac{1}{2}\left|z_{1}-z_{2}\right|$ temos que existem $\varphi_{z_{k}} \in V_{\epsilon}\left(\varphi_{z_{k}}\right), k=1,2$ e $V_{\epsilon}\left(\varphi_{z_{1}}\right) \cap V_{\epsilon}\left(\varphi_{z_{2}}\right)=\emptyset$. Agora se $z_{1}=z_{2}$ temos que $\varphi_{z_{k}}=\left(f_{k}, z_{k}, r_{k}\right), k=1,2$ satisfazendo $f_{1} \neq f_{2}$ pois $\varphi_{z_{1}}$ e $\varphi_{z_{2}}$ são distintos. Tomando $\epsilon<\min \left\{r_{1}, r_{2}\right\}$ temos $V_{\epsilon}\left(\varphi_{z_{1}}\right) \cap V_{\epsilon}\left(\varphi_{z_{2}}\right)=\emptyset$ pois em caso contrário teríamos que $\varphi_{z_{1}}$ seria continuação analítica imediata de $\varphi_{z_{1}}$, o que implicaria $f_{1}=f_{2}$ e portanto $\varphi_{z_{1}}=\varphi_{z_{2}}$.

Para mostrar que essa topologia é conexa por caminhos considere $z_{0} \in \Omega, \varphi_{z_{0}} \in M$ e $l_{k}(t), t \in[0,1], k=1,2$ dois caminhos em $\Omega$ tais que $L_{k}(0)=z_{0}$. Sejam ainda $\varphi_{t}^{k}$ elementos de funções centrados em $l_{k}(t)$ e $\varphi_{z_{k}}^{k}$ as continuações de $\varphi_{z_{0}}$ ao longo de $l_{k}(t)$. Com isso podemos definir gérmens de funções $\tilde{\varphi}_{t}$ dados por $\tilde{\varphi}_{t}=\varphi_{1-2 t}^{1}, 0 \leq t \leq \frac{1}{2}$ (volta de $\varphi_{z_{1}}$ a $\varphi_{z_{0}}$ por $l_{1}^{-1}$ ) e $\tilde{\varphi}_{t}=\varphi_{2 t-1}^{2}, \frac{1}{2} \leq t \leq 1$ (vai de $\varphi_{z_{0}}$ a $\varphi_{z_{2}}$ por $l_{2}$ ) que é uma continuação de $\varphi_{z_{1}}$ a $\varphi_{z_{2}}$ ao longo do caminho $l_{1}^{-1} \cdot l_{2}$.

A estrutura de superfície de Riemann para $M$ com essa topologia é verificada observando que se $V_{1}:=V_{\epsilon_{1}}\left(\varphi_{z_{1}}\right), V_{2}:=V_{\epsilon_{2}}\left(\varphi_{z_{2}}\right)$ e $\phi_{k}: V_{k} \rightarrow \Omega \subset \hat{\mathbb{C}}$ tal que $\phi_{k}\left(\varphi_{\bar{z}}\right)=\tilde{z}$ então $\phi_{k}\left(V_{k}\right)$ é um disco centrado em $\tilde{z}_{k}$ de raio $\epsilon_{k}$ em $\Omega$. Se $V_{1} \cap V_{2} \neq \emptyset$ então $\phi_{1} \circ \phi_{2}^{-1}: \phi_{2}\left(V_{1} \cap V_{2}\right) \rightarrow \phi_{1}\left(V_{1} \cap V_{2}\right)$ será dada por $\phi_{1} \circ \phi_{2}^{-1}(z)=z$ que é analítica e injetora e portanto um biholomorfismo. Como $M=\bigcup_{z \in \Omega} V_{\epsilon}\left(\varphi_{z}\right)$ então as funções $\phi$ do tipo acima fazem parte de um atlas que dá uma estrutura complexa para $M$.

Inspirados nos exemplos anteriores à proposição acima, podemos considerar polinômios $P(w, z)$ a duas variáveis complexas, isto é, se $(w, z) \in \mathbb{C}^{2}$ então

$$
P(w, z)=P_{0}(z) w^{n}+P_{1}(z) w^{n-1}+\ldots+P_{n}(z) .
$$

No caso em que $z=\infty$ basta considerar

$$
\tilde{P}(w, \xi)=\tilde{P}_{0}(\xi) w^{n}+\tilde{P}_{1}(\xi) w^{n-1}+\ldots+\tilde{P}_{n}(\xi)
$$


onde $\xi=1 / z$.

Definição 1.3.60. Seja $P(w, z)$ um polinômio irredutível a duas variáveis complexas como em (1.9). O conjunto dos pontos singulares $S$ de $P(w, z)$ é formado pelos $z_{0} \in \hat{\mathbb{C}}$ tais que

1. $P_{0}\left(z_{0}\right)=0$ se $z_{0} \in \mathbb{C}$ ou $\tilde{P}_{0}(0)=0$ se $z_{0}=\infty$, ou

2. se existe $w_{1} \in \mathbb{C}$ tal que $P\left(w_{1}, z_{0}\right)=\frac{\partial P}{\partial w}\left(w_{1}, z_{0}\right)=0$ quando $z_{0} \in \mathbb{C}$ ou $\tilde{P}\left(w_{1}, 0\right)=\frac{\partial \tilde{P}}{\partial w}\left(w_{1}, 0\right)=0$ se $z_{0}=\infty$,

onde $\tilde{P}(w, \xi)$ e $\tilde{P}_{0}(\xi)$ são como em (1.10).

Proposição 1.3.61. Seja $P(w, z)$ um polinômio irredutivel como em (1.9) e $S$ seu conjunto de pontos singulares. Então para todo $z \in \hat{\mathbb{C}} \backslash S$ existem $n$ raízes distintas $w_{k}=w_{k}(z), k=1, \ldots, n$, de $P(w, z)=0$.

Demonstração: Para cada $z_{0} \in \hat{\mathbb{C}} \backslash S$ a equação (1.9) é um polinômio de grau $n$ na variável $w$ que se fatora na forma

$$
P\left(w, z_{0}\right)=\left(w-w_{1}\right)^{k_{1}} \ldots\left(w-w_{m}\right)^{k_{m}}
$$

onde cada $w_{i}$ é tal que $P\left(w_{i}, z_{0}\right)=0$. Mas como $z_{0}$ é não-singular então $\frac{\partial P}{\partial w}\left(w_{i}, z_{0}\right) \neq 0$ donde $k_{i}=1$ e portanto $m=n$.

Vamos agora mostrar que as soluções $w_{k}(z)$ podem ser agrupadas em $n$ funções analíticas de $z$ distintas numa vizinhança de cada $z_{0} \in \hat{\mathbb{C}} \backslash S$. Estas funções representarão localmente os diversos ramos da função algébrica globalmente definida por $P(w, z)=0$.

Lema 1.3.62. Seja $f(z)$ uma função analítica num domínio de $\mathbb{C}$. Suponha que $z=$ $x+i y, x, y \in \mathbb{R}$ e $f(z)=u(x, y)+i v(x, y)$ (identificando aqui $x+i y \operatorname{com}(x, y) \in \mathbb{R}^{2}$ ), onde $u, v$ são funções a valores reais. Valem então as seguintes igualdades:
(a) $f_{z}:=\frac{\partial f}{\partial z}=2 u_{z}$
(b) $f_{z}=2 i v_{z}$
(c) $f_{z}=f_{x}$
(d) $f_{z}=-i f_{y}$

\section{Demonstração:}


(a)

$$
\begin{aligned}
f_{z}=\frac{1}{2}\left(f_{x}-i f_{y}\right) & =\frac{1}{2}\left(u_{x}+i v_{x}-i\left(u_{y}+i v_{y}\right)\right)=\frac{1}{2}\left(u_{x}-i u_{y}+v_{y}-i u_{y}\right)= \\
& =\frac{1}{2}\left(u_{x}-i u_{y}+u_{x}-i u_{y}\right)=u_{x}-i u_{y}=2 u_{z} .
\end{aligned}
$$

(b)

$$
\begin{aligned}
f_{z}=\frac{1}{2}\left(f_{x}-i f_{y}\right) & =\frac{1}{2}\left(u_{x}+i v_{x}-i\left(u_{y}+i v_{y}\right)\right)=\frac{1}{2}\left(v_{y}+i v_{x}+v_{y}+i v_{x}\right)= \\
& =v_{y}+i v_{x}=i\left(v_{x}-i v_{y}\right)=2 i v_{z} .
\end{aligned}
$$

(c)

$$
\begin{aligned}
f_{z}=\frac{1}{2}\left(f_{x}-i f_{y}\right) & =\frac{1}{2}\left(u_{x}+i v_{x}-i\left(u_{y}+i v_{y}\right)\right)=\frac{1}{2}\left(u_{x}+i v_{x}+u_{x}+i v_{x}\right)= \\
& =u_{x}+i v_{x}=f_{x}
\end{aligned}
$$

(d)

$$
\begin{aligned}
f_{z}=\frac{1}{2}\left(f_{x}-i f_{y}\right) & =\frac{1}{2}\left(u_{x}+i v_{x}-i\left(u_{y}+i v_{y}\right)\right)=\frac{1}{2}\left(v_{y}-i u_{y}+v_{y}-i u_{y}\right)= \\
& =v_{y}-i u_{y}=-i\left(u_{y}+i v_{y}\right)=-i f_{y} .
\end{aligned}
$$

Teorema 1.3.63. Sejam $P(w, z)$ um polinômio irredutível, $S$ seu conjunto de pontos singulares e $z_{0} \in \hat{\mathbb{C}} \backslash S$. Então existe $V_{z_{0}}$ vizinhança aberta de $z_{0}$ e $n$ funções analíticas $w_{k}(z), k=1, \ldots, n$ tais que $P\left(w_{k}(z), z\right)=0$ e $w_{i}(z) \neq w_{j}(z)$ se $i \neq j, \forall z \in V_{z_{0}}$.

Demonstração: Seja $z_{0} \in \hat{\mathbb{C}} \backslash S, z_{0} \neq \infty$. Sejam também $w_{1}, \ldots, w_{n}$ as $n$ raízes distintas de $P\left(w, z_{0}\right)$. Como $\frac{\partial P}{\partial w}\left(w, z_{0}\right) \neq 0$ existem vizinhanças $V_{1}, \ldots, V_{n}$ de $z_{0}$ em $\mathbb{C}$ com $V_{k} \cap S=\emptyset$ e $W_{k}$ vizinhanças de $w_{k}$ e funções $w_{k}(z): V_{k} \rightarrow W_{k}$, com $w_{k}\left(z_{0}\right)=w_{k}$ tais que $\left(w_{k}(z), z\right) \in W_{k} \times V_{k}$ são todas as soluções de $P(w, z)=0$ em $W_{k} \times V_{k}, k=1, \ldots, n$. Então para $z_{0} \in V_{z_{0}}:=V_{1} \cap V_{2} \cap \ldots \cap V_{n}$ temos que $\left(w_{k}(z), z\right)$ é um sistema completo de soluções de $P(w, z)=0$. Vamos agora mostrar que cada $w_{k}(z)$ é função analítica de $z$ em $V_{z_{0}}$. Se escrevemos $w_{k}(z)=u_{k}(z)+i v_{k}(z)$ e $z=x+i y$ temos

$$
P\left(w_{k}(z), z\right)=0 \Longleftrightarrow F\left(w_{k}(z), z\right)+i G\left(w_{k}(z), z\right)=0, z \in V_{z_{0}},
$$

onde $F(w, z)$ e $G(w, z)$ são respectivamente as partes real e imaginária de $P(w, z)$. Assim temos

$$
P\left(w_{k}(z), z\right)=0 \Longleftrightarrow F\left(w_{k}(z), z\right)=G\left(w_{k}(z), z\right)=0
$$


ou seja,

$$
\begin{aligned}
& F\left(u_{k}+i v_{k}, x+i y\right)=0 \\
& G\left(u_{k}+i v_{k}, x+i y\right)=0 .
\end{aligned}
$$

Identificando $u+i v \in \mathbb{C}$ com $(u, v) \in \mathbb{R}^{2}, x+i y \in \mathbb{C}$ com $(x, y) \in \mathbb{R}^{2}$ e derivando (1.11) e (1.12) em $x$ e $y$ no ponto $z_{0}=x_{0}+i y_{0}$ temos

$$
\begin{aligned}
& \frac{\partial F}{\partial x}(u, v, x, y)=0 \Rightarrow u_{x} F_{u}+v_{x} F_{v}+F_{x}=0 \\
& \frac{\partial F}{\partial y}(u, v, x, y)=0 \Rightarrow u_{y} F_{u}+v_{y} F_{v}+F_{y}=0 \\
& \frac{\partial G}{\partial x}(u, v, x, y)=0 \Rightarrow u_{x} G_{u}+v_{x} G_{v}+G_{x}=0 \\
& \frac{\partial G}{\partial y}(u, v, x, y)=0 \Rightarrow u_{y} G_{u}+v_{y} G_{v}+G_{y}=0 .
\end{aligned}
$$

Agora, como $F$ e $G$ dependem analiticamente de $w$ temos $F_{u}=G_{v}, F_{v}=-G_{u}$, e além disso dependem também analiticamente de $x$ e $y$ donde $F_{x}=G_{y}, F_{y}=-G_{x}$. Disto e da multiplicação (1.14) e (1.16) por $i$ somadas respectivamente a (1.13) e a (1.15), temos

$$
\begin{aligned}
& u_{x}\left(F_{u}+i G_{u}\right)+v_{x}\left(F_{v}+i G_{v}\right)+F_{x}+i G_{x}=0 \Rightarrow \\
& u_{x}\left(F_{u}-i F_{v}\right)+i v_{x}\left(F_{u}-i F_{v}\right)+F_{x}+i G_{x}=0,
\end{aligned}
$$

e

$$
\begin{aligned}
& u_{y}\left(F_{u}+i G_{u}\right)+v_{y}\left(F_{v}+i G_{v}\right)+F_{y}+i G_{y}=0 \Rightarrow \\
& u_{y}\left(F_{u}-i F_{v}\right)+i v_{y}\left(F_{u}-i F_{v}\right)+F_{y}+i G_{y}=0
\end{aligned}
$$

e, do lema (1.3.62), vem que

$$
\begin{aligned}
u_{x} \frac{1}{2} \frac{\partial P}{\partial w}+i v_{x} \frac{1}{2} \frac{\partial P}{\partial w}+\frac{\partial P}{\partial x} & =0 \Rightarrow \\
\frac{1}{2} \frac{\partial P}{\partial w} w_{x}+\frac{\partial P}{\partial z} & =0
\end{aligned}
$$

e 


$$
\begin{aligned}
u_{y} \frac{1}{2} \frac{\partial P}{\partial w}+i v_{y} \frac{1}{2} \frac{\partial P}{\partial w}+\frac{\partial P}{\partial y} & =0 \Rightarrow \\
\frac{1}{2} \frac{\partial P}{\partial w} w_{y}+2 i \frac{\partial P}{\partial z} & =0
\end{aligned}
$$

ou seja,

$$
\begin{aligned}
2 \frac{\partial P}{\partial z} & =-\frac{\partial P}{\partial w} w_{x} \mathrm{e} \\
2 \frac{\partial P}{\partial z} & =i w_{y} \frac{\partial P}{\partial w}
\end{aligned}
$$

De (1.17) e (1.18) temos $\frac{\partial P}{\partial w}\left(w_{x}+i w_{y}\right)=0$. Como $z$ está numa vizinhança de pontos regulares de $z_{0}$ temos $w_{x}+i w_{y}=0 \Rightarrow \frac{\partial w}{\partial \bar{z}}=0$, ou seja, $w(z)$ é analítica.

Se $z_{0}=\infty$ consideramos o polinômio $\tilde{P}(w, \xi)$, com $\xi=1 / z$. Como visto acima temos $n$ soluções distintas $\tilde{w}_{k}(\xi), k=1, \ldots, n$ numa vizinhança de $\xi=0$. Logo $w_{k}(z)=\tilde{w}_{k}(\xi)$ são as soluções analíticas de $P(w, z)=0$ numa vizinhança de $z_{0}=\infty$.

Com o teorema acima, temos que a um polinômio irredutível $P(w, z)$ está associada uma superfície de Riemann $M$ onde existem soluções $(w(z), z)$ de $P(w, z)$, se $z \in \hat{\mathbb{C}} \backslash S$. Assim, $M$ é um recobrimento de $n$ folhas de $\hat{\mathbb{C}} \backslash S$, se o grau de $P(w, z)$ é $n$. Podemos estender $M$ de modo que ela contenha os pontos críticos em sua estrutura. Para isso, consideremos todas continuações analíticas dos gérmens definidos por cada $w_{i}$ e com raio tal que o disco não contenha nenhum ponto crítico de $P(w, z)$ ao longo de curvas fechadas que contenham somente um ponto crítico de $P(w, z)$. Podemos verficar que somando o menor número de voltas necessário para que cada gérmen em torno de cada ponto crítico retorne ao valor original resulta sempre em $n$, nos pontos regulares isso já acontecia pois o número de folhas é constante. Observe que se após uma volta em torno de um ponto crítico $z_{s}$ de um certo gérmen dado por $w_{i}(z)$ ele não se altera então temos que $w_{i}$ é holomorfa em $z_{s}$. A figura 1.1 representa esta situação, onde numa vizinhança de $z_{1}$ o gérmem dado pela solução $w_{1}(z)$ se repete após três voltas e o gérmem dado por $w_{2}(z)$ se repete após duas voltas. Assim, acrescentamos à superfície $M$ os pontos correspondentes a cada diferente gérmen obtido pela continuação de cada $w_{i}$ em torno de cada ponto singular $z_{s}$, chamando-a de $\hat{M}$. Para a construção da topologia em $\hat{M}$ ver [4].

Teorema 1.3.64. A superfície de Riemann $\hat{M}$ associada ao polinômio $P(w, z)$ descrita no parágrafo anterior é compacta. 


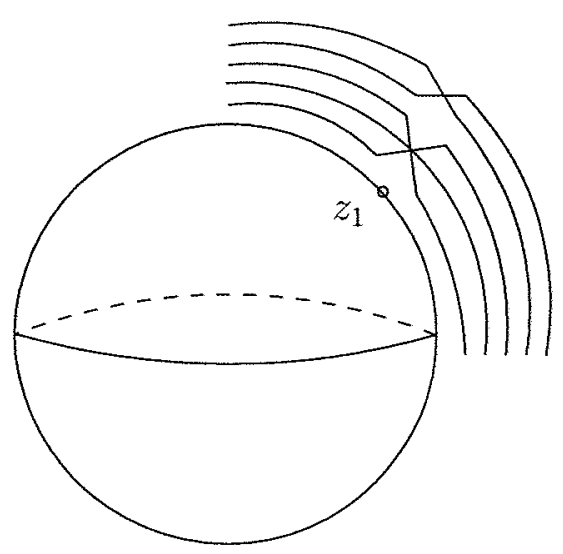

Figura 1.1: Vizinhança de um ponto de ramificação.

Demonstração: Ver [4].

Existe ainda uma recíproca para esse teorema:

Teorema 1.3.65. Seja $M$ uma superfície de Riemann compacta. Então existe um polinômio irredutivel $P(w, z)$ tal que a superfície de Riemann associada a ele é conformemente equivalente $a M$.

Demonstração: Ver [2].

Como vimos no parágrafo após o teorema 1.3 .4 cada função $w_{i}(z)$ solução de $P(w, z)=0$ tem comportamento local do tipo $w_{i}(z)=z^{p_{k}}$ e das considerações acima temos que $\sum_{i} p_{k}=n$. Generalizando conceito da definição 1.3 .5 temos que o índice de ramificação de $z$ ponto singular de $P(w, z)$ tal que $w_{i}(z)=0^{\left(p_{k}\right)}$ é dado por $i(z):=\sum_{i}\left(p_{k}-1\right)$.

Definição 1.3.66. Seja $\hat{M}$ uma superfície de Riemann compacta. A sua ordem de ramificação total é dada por

$$
b(\hat{M}):=\sum_{z \in \hat{M}} i(z)
$$

Observe que se $z$ é um ponto não-singular de $P(w, z)$ então $i(z)=0$, donde a soma acima é finita, pois os pontos críticos são finitos.

Proposição 1.3.67 (Fórmula de Riemann-Hurwitz). Seja $\hat{M}$ uma superfície de Riemann compacta, dada por um polinômio $P(w, z)$ irredutivel de grau $n$ em $w$. Então o gênero $\gamma$ de $\hat{M}$ é

$$
\gamma=1-n+\frac{b(\hat{M})}{2}
$$


Demonstração: Ver [4].

Existe uma generalização deste resultado para um recobrimento ramificado entre duas superfícies de Riemann:

Proposição 1.3.68. Seja $\pi: M \rightarrow N$ um recobrimento holomorfo ramificado entre duas superficies de Riemann compactas $M$ e $N$ e sejam $b$ a ordem de ramificação total de $\pi, \gamma$ e $\gamma^{\prime}$ os gêneros de $M$ e $N$ respectivamente. Então

$$
\gamma=1+n\left(\gamma^{\prime}-1\right)+\frac{b}{2}
$$

Demonstração: Ver [8].

Note que a proposição 1.3 .67 é consequência direta da proposição acima quando $N=\hat{\mathbb{C}}$, pois nesse caso $\gamma^{\prime}=0$.

\subsection{Superfícies Mínimas e a representação de Wei- erstrass}

Definição 1.4.1. Uma superfície $S=f(M)$ é mínima se $\vec{H} \equiv \overrightarrow{0}$.

Da definição e da equação (1.7) observamos que se $S$ é uma superfície mínima e $u, v$ são coordenadas isotérmicas para $S$ temos $f_{u u}+f_{v v} \equiv 0$, ou seja, as funções coordenadas de $S$ são funções harmônicas de $\mathbb{R}^{2}$ em $\mathbb{R}$.

Desta observação temos o seguinte corolário:

Corolário 1.4.2. Não existe superfície mínima compacta em $\mathbb{R}^{3}$.

Demonstração: Seja $S=f(M)$ tal superfície. Se $S$ é compacta então $M$ é compacta e portanto cada coordenada de $f:=\left(f_{1}, f_{2}, f_{3}\right)$ assume um valor máximo em um $p_{i} \in M, i=1 \ldots 3$. Pelo princípio do máximo para funções harmônicas reais cada $f_{i}, 1 \leq i \leq 3$ seria constante numa vizinhança de $p_{i}$, o que implica $f$ constante em $M$. Contradição, pois $f$ é imersão.

Vamos agora construir a representação de Weierstrass para superfícies mínimas. Esta é a ferramenta fundamental para a construção e estudo de exemplos de superfícies mínimas, pois os dados de entrada desta representação tem relação muito forte com a geometria extrínseca da superfície no $\mathbb{R}^{3}$ (especialmente a aplicação normal como veremos) e, por outro lado esses dados definem a equação algébrica da superfície de Riemann que será imersa no $\mathbb{R}^{3}$.

Proposição 1.4.3. Se $S=f(M)$ é uma superfície conforme do $\mathbb{R}^{3}$ então $\partial f:=f_{w} d w$ $e \bar{\partial} f:=f_{\bar{w}} d \bar{w}$ onde $d w=d u+i d v e d \bar{w}=d u-i d v \operatorname{para}(U, w=u+i v) \in \mathcal{H}(M)$ satifazem: 
(a) $\partial f$ e $\bar{\partial} f$ são 1-formas a valores em $\mathbb{C}^{3}$ globalmente definidas,

(b) $\overline{\partial f}=\bar{\partial} f$,

(c) $\langle\partial f, \partial f\rangle^{\mathbb{C}} \equiv 0$,

(d) S é mínima se e somente se af é holomorfa.

\section{Demonstração:}

(a) Se $(U, w=u+i v),(V, z=x+i y) \in \mathcal{H}(M)$ com $U \cap V \neq \emptyset$ temos

$$
f_{z}=f_{w} \frac{d w}{d z} \text { e } f_{\bar{z}}=f_{\bar{w}} \overline{\left(\frac{d w}{d z}\right)}=f_{\bar{w}} \frac{d \bar{w}}{d \bar{z}}
$$

e portanto $f_{z} d z=f_{w} d w$ e $f_{\bar{z}} d \bar{z}=f_{\bar{w}} d \bar{w}$ ao longo de $U \cap V$.

(b) $\overline{\partial f}=\overline{f_{w} d w}=\overline{f_{w} d w}=\frac{1}{2} \overline{\left(f_{u}-i f_{v}\right)(d u+i d v)}=\frac{1}{2}\left(f_{u}+i f_{v}\right)(d u-i d v)=$ $f_{\bar{w}} d \bar{w}=\bar{\partial} f$.

(c) $\langle\partial f, \partial f\rangle^{\mathbb{C}}=\left\langle f_{w}, f_{w}\right\rangle^{\mathbb{C}}=\left\langle f_{u}+i f_{v}, f_{u}+i f_{v}\right\rangle=\left\langle f_{u}, f_{u}\right\rangle-\left\langle f_{v}, f_{v}\right\rangle+2 i\left\langle f_{u}, f_{v}\right\rangle \equiv 0$, pois $u$ e $v$ são coordenadas isotérmicas.

(d) $S$ é mínima $\Longleftrightarrow \vec{H} \equiv \overrightarrow{0} \Longleftrightarrow f_{w \bar{w}} \equiv 0 \Longleftrightarrow f_{w}$ é holomorfa $\Longleftrightarrow \partial f$ é holomorfa.

Proposição 1.4.4. Se $S=f(M)$ é uma superfície conforme mínima do $\mathbb{R}^{3},(U, w=$ $u+i v) \in \mathcal{H}(M)$ com $w_{0} \in w(U)$ e $\tilde{f}:=f \circ w^{-1}: \mathbb{C} \rightarrow \mathbb{R}^{3}$ então

$$
\tilde{f}(w)=\tilde{f}\left(w_{0}\right)+2 \Re \int_{w_{0}}^{w} \partial f .
$$

Demonstração: $2 \partial f=\left(f_{u}-i f_{v}\right)(d u+i d v)=\left(f_{u} d u+f_{v} d v\right)+i\left(f_{u} d v-f_{v} d u\right)$. Se $\gamma$ é um caminho de classe $C^{1}$ por partes em $M$ tal que $w(\gamma(t))=u(t)+i v(t), t \in[a, b]$ $\operatorname{com} \gamma(a)=w_{0}$ e $\gamma(b)=w$ temos

$$
\begin{aligned}
\int_{\gamma} f_{w} d w & =\int_{a}^{b} f_{w}(\gamma(t)) \gamma^{\prime}(t) d t=\int_{a}^{b} \frac{1}{2}\left(f_{u}(\gamma(t))-i f_{v}(\gamma(t))\right)\left(u^{\prime}(t)+i v^{\prime}(t)\right) d t \\
& =\frac{1}{2} \int_{a}^{b} \frac{d}{d t} f(\gamma(t)) d t+\frac{i}{2} \int_{a}^{b}\left(f_{u}(\gamma(t)) v^{\prime}(t)+f_{v}(\gamma(t)) u^{\prime}(t)\right) d t
\end{aligned}
$$

Logo, $2 \Re \int_{\gamma} f_{w} d w=\int_{a}^{b} \frac{d}{d t} f(\gamma(t)) d t=\tilde{f}(\gamma(b))-\tilde{f}(\gamma(a))=\tilde{f}(w)-\tilde{f}\left(w_{0}\right)$.

O resultado a seguir permite a construção da superfície conjugada a $S=f(M)$. 
Proposição 1.4.5. Se $S=f(M)$ é uma superfície conforme mínima e $(U, w=$ $u+i v) \in \mathcal{H}(M)$ com $U$ simplesmente conexo então

(a) existe $\tilde{f}^{*}: w(U) \subset \mathbb{C} \rightarrow \mathbb{R}^{3}$ harmônica, única a menos de constante do $\mathbb{R}^{3}$, tal que $\Phi:=\tilde{f}+i \tilde{f}^{*}: w(U) \rightarrow \mathbb{C}^{3}$ tem coordenadas holomorfas $\left(\tilde{f}:=f \circ w^{-1}\right)$.

(b) $S^{*}:=\tilde{f}^{*}(w(U))$ é uma superfície conforme minima do $\mathbb{R}^{3}$.

(c) Se $w_{0} \in w(U)$ então

$$
\tilde{f}^{*}(w)=\tilde{f}^{*}\left(w_{0}\right)+2 \Im \int_{w_{0}}^{w} \frac{1}{i} f_{w} d w
$$

\section{Demonstração:}

(a) Vem da existência da conjugada harmônica de uma função harmônica em domínios abertos simplesmente conexos de $\mathbb{C}$.

(b) As equações de Cauchy-Riemann para $\Phi, \tilde{f}_{u}=\tilde{f}_{v}^{*}$ e $\tilde{f}_{v}=-\tilde{f}_{u}^{*}$ implicam

$$
\tilde{f}_{u u}^{*}+\tilde{f}_{v v}^{*}=-\tilde{f}_{v u}+\tilde{f}_{u v} \equiv 0 \text {. }
$$

Logo $\tilde{f}^{*}$ é harmônica em $w(U)$ e portanto $S^{*}$ é mínima conforme.

(c) Demonstração análoga à da Proposição 1.4.4.

Definição 1.4.6. A superfície $S^{*}$ da proposição acima é chamada superfície mínima conjugada da superfície $S=f(U)$.

A superfície mínima conjugada apresenta propriedades geométricas, intrínsecas e extrínsecas, fortemente relacionadas com as da superfície original.

Proposição 1.4.7. Se $\alpha, \beta$ são duas 1-formas ao longo de uma superfície de Riemann $M$ dadas localmente por $\alpha=f d w$ e $\beta=g d w$ então $\frac{\alpha}{\beta}=\frac{f}{g}$ é uma função meromorfa em $M$ globalmente definida.

Demonstração: $\operatorname{Sejam~}(U, w),(V, z) \in \mathcal{H}(M) \operatorname{com} U \cap V \neq \emptyset$. Então

$$
\begin{aligned}
& \alpha=f d w=\tilde{f} d z \\
& \beta=g d w=\tilde{g} d z
\end{aligned}
$$


Logo $\frac{d w}{d z}=\frac{\tilde{f}}{f}=\frac{\tilde{g}}{g} \Rightarrow \frac{f}{g}=\frac{\tilde{f}}{\tilde{g}}$ ao longo de $U \cap V$.

Se $S=f(M)$ é superfície mínima conforme do $\mathbb{R}^{3}$ com $f=\left(f_{1}, f_{2}, f_{3}\right)$ então $\partial f$ é uma 1-forma holomorfa pois $f_{w \bar{w}} \equiv 0$ em qualquer sistema de coordenadas $(U, w=u+i v)$. Logo, do item(c) da proposição $1.4 .3\langle\partial f, \partial f\rangle^{\mathbb{C}} \equiv 0$ e portanto

$$
\begin{aligned}
0 \equiv\langle\partial f, \partial f\rangle^{\mathbb{C}}= & \left\langle\left(\left(f_{1}\right)_{u},\left(f_{2}\right)_{u},\left(f_{3}\right)_{u}\right)-i\left(\left(f_{1}\right)_{v},\left(f_{2}\right)_{v},\left(f_{3}\right)_{v}\right)\right. \\
& \left.\left(\left(f_{1}\right)_{u},\left(f_{2}\right)_{u},\left(f_{3}\right)_{u}\right)-i\left(\left(f_{1}\right)_{v},\left(f_{2}\right)_{v},\left(f_{3}\right)_{v}\right)\right\rangle \\
= & \sum_{k=1}^{3}\left(f_{k}\right)_{u}^{2}-\sum_{k=1}^{3}\left(f_{k}\right)_{v}^{2}-2 i \sum_{k=1}^{3}\left(f_{k}\right)_{u}\left(f_{k}\right)_{v} \\
= & \sum_{k=1}^{3}\left[\left(f_{k}\right)_{u}^{2}-\left(f_{k}\right)_{v}^{2}-2 i\left(f_{k}\right)_{u}\left(f_{k}\right)_{v}\right] \\
= & \sum_{k=1}^{3}\left[\left(f_{k}\right)_{u}-i\left(f_{k}\right)_{v}\right]^{2} \\
= & \left(\partial f_{1}\right)^{2}+\left(\partial f_{2}\right)^{2}+\left(\partial f_{3}\right)^{2}
\end{aligned}
$$

Logo $\left(\partial f_{1}\right)^{2}+\left(\partial f_{2}\right)^{2}+\left(\partial f_{3}\right)^{2} \equiv 0$ e se $\partial f_{3} \not \equiv 0$ (ie, a terceira coordenada de $f$ não é constante) temos

$$
\left(-\frac{\partial f_{1}}{\partial f_{3}}-i \frac{\partial f_{2}}{\partial f_{3}}\right)\left(\frac{\partial f_{1}}{\partial f_{3}}-i \frac{f_{2}}{\partial f_{3}}\right) \equiv 1 \text {. }
$$

Então da proposição 1.4 .7 temos que $g:=\left(-\frac{\partial f_{1}}{\partial f_{3}}-i \frac{\partial f_{2}}{\partial f_{3}}\right)$ é uma função meromorfa sobre $M$ e $\frac{1}{g}=\left(\frac{\partial f_{1}}{\partial f_{3}}-i \frac{f_{2}}{\partial f_{3}}\right)$. Das definições de $g$ e $\frac{1}{g}$ temos

$$
\frac{\partial f_{2}}{\partial f_{3}}=\frac{i}{2}\left(g+\frac{1}{g}\right) \text { e } \frac{\partial f_{1}}{\partial f_{3}}=\frac{i}{2}\left(g+\frac{1}{g}\right) .
$$

Assim $\partial f=\left(\frac{1}{2}\left(\frac{1}{g}-g\right), \frac{i}{2}\left(\frac{1}{g}+g\right), 1\right) \partial f_{3}$

O resultado a seguir relaciona a função $g$ definida acima com a aplicação normal de Gauss de uma superfície mínima.

Teorema 1.4.8. Sejam $\hat{\mathbb{C}}:=\mathbb{C} \cup\{\infty\}$ a esfera de Riemann e st $: \mathbb{C} \rightarrow \hat{\mathbb{C}}$ é projeção estereográfica dada por

$$
s t(w)=\left(\frac{2 \Re w}{|w|^{2}+1}, \frac{2 \Im w}{|w|^{2}+1}, \frac{|w|^{2}-1}{|w|^{2}+1}\right)
$$


quando $w \in \mathbb{C}$ e st $(\infty)=(0,0,1)$. Se $S=f(M) \in \mathbb{R}^{3}, f=\left(f_{1}, f_{2}, f_{3}\right)$ é uma superfície minima conforme defina $g \equiv \infty$ se $\partial f_{3} \equiv 0$ e $g=\left(-\frac{\partial f_{1}}{\partial f_{3}}-i \frac{\partial f_{2}}{\partial f_{3}}\right)$ em caso contrário. Então, stog é a aplicação normal de Gauss de $S$.

Demonstração: Segue do fato que em coordenadas locais $z=u+i v$ temos

$$
f_{u} \times f_{v}=-\frac{1}{4} \Re \partial f \times \Im \partial f
$$

donde $N=\frac{2}{1+|g|^{2}}\left(\Re g, \Im g, \frac{|g|^{2}-1}{2}\right)$.

O teorema a seguir dá a representação de Weierstrass de uma superfície mínima do $\mathbb{R}^{3}$.

Definição 1.4.9. Seja $S=f(M)$ um superfície mínima. Sua curvatura total é dada por $\int_{M} K d M$, onde $K$ é a curvatura gaussiana de $S$ definida em 1.2 .4 e $d M$ é o elemento de área de $M$.

Teorema 1.4 .10 (Representação de Weierstrass). Sejam $M$ superfície de Riemann e $S=f(M)$ superficie minima completa com curvatura total finita do $\mathbb{R}^{3}$. Então

(a) $M$ é conformemente equivalente a uma superfície de Riemann $\bar{M}_{\gamma}$ de gênero $\gamma$ menos um número finito de pontos, ie, $M \cong \bar{M}_{\gamma} \backslash\left\{q_{1}, \ldots, q_{N}\right\}$.

(b) Existem $(g, d H)$ respectivamente uma função e uma diferencial meromorfas sobre $\bar{M}_{\gamma}$, dH analitica sobre $M$ tais que $g:=s t^{-1} \circ N$, onde $N: M \rightarrow \hat{\mathbb{C}}$ é a aplicação normal de Gauss de $S$ e

$$
f(p)=f\left(p_{0}\right)+2 \Re \int_{p_{0}}^{p}\left(\frac{1}{2}\left(\frac{1}{g}-g\right), \frac{i}{2}\left(\frac{1}{g}+g\right), 1\right) d H, p_{0} \in M
$$

Reciprocamente, sejam $M$ uma superficie de Riemann como a do item (a) acima, $p, p_{0} \in M, g: M \rightarrow \hat{\mathbb{C}}$ e $d H$ respectivamente uma função meromorfa não constante $e$ uma diferencial meromorfa sobre $\bar{M}_{\gamma}$ tais que $d H$ é analítica sobre $M e$

(a') $\alpha:=\left(\frac{1}{2}\left(\frac{1}{g}-g\right), \frac{i}{2}\left(\frac{1}{g}+g\right), 1\right)$ dH é uma diferencial holomorfa definida em $M$;

(b') $\alpha$ não tem períodos reais, ie, $\Re \int_{\gamma} \alpha=0$, para todo $\gamma$ caminho fechado de $M$;

(c) $\langle\alpha, \bar{\alpha}\rangle^{\mathrm{C}}>0$ 
Então, para todo $c \in \mathbb{R}^{3}$

$$
f(p):=\Re \int_{p_{0}}^{p} \alpha+c
$$

está bem definida e $S=f(M)$ é uma imersão conforme minima em $\mathbb{R}^{3}$.

Demonstração: A os itens (a'), (b') e (c') são consequência da proposição 1.4 .4 e das observações após a proposição 1.4.7. O outro lado pode ser encontrado em [12].

Definição 1.4.11. O par $(g, d H)$ do teorema acima é chamado par de Weierstrass ou dados de Weierstrass de $S=f(M)$.

Do teorema acima notamos que toda superfície mínima completa com curvatura total finita é a imersão de uma superfície de Riemann menos um número finito de pontos. Se $f$ e $f^{*}$ são as representações de Weierstrass de uma superfície mínima e sua conjugada, então $f+i f^{*}$ é uma aplicação de recobrimento de $M$ em $\mathbb{C}$ e a função $g$ do par de Weierstrass também é uma aplicação de recobrimento de $\bar{M}_{\gamma}$ sobre $\hat{\mathbb{C}}$.

Definição 1.4.12. Sejam $M=\bar{M}_{\gamma} \backslash\left\{q_{1}, \ldots, q_{N}\right\}$ uma superfície de Riemann e $S=$ $f(M)$ uma imersão mínima completa de $M$ em $\mathbb{R}^{3}$. Um fim de $S$ é a imagem por $f$ de uma vizinhança $V$ de $q_{i}$ em $\bar{M}_{\gamma}$ de modo que $V \cap\left(\left\{q_{1}, \ldots, q_{N}\right\}-q_{i}\right)=\emptyset$.

Proposição 1.4.13. Seja $S=f(M)$ uma superfície mínima completa em $\mathbb{R}^{3}$, onde $M=\bar{M}_{\gamma} \backslash\left\{q_{1}, \ldots, q_{N}\right\}$ e $(g, d H)$ seu par de Weierstrass. Então

$$
\operatorname{deg}(g)=\gamma+N-1
$$

Demonstração: Ver [9].

Temos a seguinte expressão para métrica de uma superfície mínima completa em termos dos seus dados de Weierstrass:

Proposição 1.4.14. Seja $S=f(M)$ uma superfície mínima completa em $\mathbb{R}^{3} e$ $(g, d H)$ seu par de Weierstrass. Então a sua métrica é dada por

$$
d s^{2}=\frac{1}{2}\left(\frac{1}{|g|}+|g|\right)^{2}|d H|^{2} .
$$

Demonstação: Basta fazer os cáculos diretamente lembrando que se $z=u+i v$ são coordenadas isotérmicas, então localmente $\partial f=f_{z} d z$, e que $f_{z}=f_{u}$ (lema 1.3.62) donde $\lambda^{2}=\left\langle f_{u}, f_{u}\right\rangle=\left\langle f_{z}, \overline{f_{z}}\right\rangle^{\mathbb{C}}$.

Proposição 1.4.15. Nas mesmas condições da proposição acima temos, que se $K$ é a curvatura Gaussiana de $S=f(M)$ com dados de Weierstrass $(g, d H)$ então

$$
K=\frac{-16}{\left(|g|+|g|^{-1}\right)^{4}}\left|\frac{d g / g}{d H}\right|^{2} .
$$


Demonstração: Novamente basta fazer os cálculos diretamente lembrando que podemos calcular $K=\frac{b_{11} b_{22}-b_{12}^{2}}{\lambda^{4}}$ usando as expressões da proposição 1.2 .11 e que ao escrevermos $d H=h d w$ num sistema de coordenadas $(U, w)$ de $\bar{M}_{\gamma}$ para $h$ função meromorfa temos

$$
\left\langle f_{w w}, N\right\rangle=-\frac{g_{w} h}{g}
$$

Em termos dos dados de Weierstrass de uma superfície temos as seguintes relações para a segunda forma fundamental:

Proposição 1.4.16. Seja $S=f(M)$ uma superficie minima com par de Weierstrass $(g, d H)$ e sejam $B$ e $B^{\mathbb{C}}$ sua segunda forma fundamental e a segunda forma fundamental complexificada. Então

(a) $B^{2,0}=-\frac{d g}{g} d H$;

(b) $B=-2 \Re\left(\frac{d g}{g} d H\right)$.

Demonstração: A prova de (a) segue da definição 1.2 .10 e da expressão para $\left\langle f_{w w}, N\right\rangle$ da demonstração da proposição 1.4.15. Para provar (b), basta observar que $B=2 \Re B^{2,0}=-2 \Re\left(\frac{d g}{g} d H\right)$. Para verificar isso podemos escrever $B^{2,0}$ fazendo uso das expressões de 1.2 .10 e de 1.2.11.

Os teoremas a seguir impõe limitações sobre o gênero e os tipos de fins de uma superfície mínima mergulhada em $\mathbb{R}^{3}$.

Teorema 1.4.17 (López-Ros). Seja $S$ uma superfície mínima completa não planar de curvatura total finita mergulhada em $\mathbb{R}^{3}$. Se $S$ é uma imersão da esfera então $S$ é o catenóide.

Demonstração: Ver [5].

Teorema 1.4.18 (Schöen). Seja S uma superfície minima completa de curvatura total finita mergulhada em $\mathbb{R}^{3}$ comdois fins. Então $S$ é o catenóide.

Demonstração: Ver [13]. 


\subsection{Princípios de simetria para superfícies míni- mas}

Como vimos na seção anterior, a representação de Weierstrass de uma superfície mínima em $\mathbb{R}^{3}$ é dada por um integrando holomorfo e vamos explorar esse fato tentando extrair algumas simetrias para a imagem da imersão baseados no princípio de simetria de Schwarz para funções holomorfas que enunciamos a seguir.

Definição 1.5.1. Sejam $w, \tilde{w} \in \hat{\mathbb{C}}$.

(a) Se $C$ é uma circunferência em $\mathbb{C}$ de centro $a$ e raio $r$, então $\tilde{w}$ é o simétrico de $w$ se $\tilde{w}=a+\frac{r^{2}}{\bar{w}-\bar{a}}$. Podemos então definir a simetria $S(w)=a+\frac{r^{2}}{\bar{w}-\bar{a}}$ relativa à circunferência $C$.

(b) Se $L$ é uma reta de $\mathbb{C}$ paralela a $b=|b| e^{i \theta} \neq 0$ passando por $a$, então $\tilde{w}$ é o simétrico de $w$ se $\tilde{w}=e^{2 i \theta}(\bar{w}-\bar{a})+a$. Podemos então definir a simetria $T(w)=e^{2 i \theta}(\bar{w}-\bar{a})+a$ relativa à reta $L$.

Teorema 1.5.2 (Princípio de Simetria de Schwarz). Sejam $C_{i}$ uma circunferência ou reta de $\mathbb{C}$ e $S_{i}$ as simetrias relativas a $C_{i}$ respectivamente, $i=1,2$. Seja $G$ aberto conexo de $\mathbb{C}$ tal que $G \cap C_{1}=\emptyset$, FrG $\cap C_{1}=C_{0}$, onde $C_{0}$ é um arco de $C_{1}$. Seja ainda $f$ holomorfa em $G$, contínua no bordo de $G$, com $f\left(C_{0}\right) \subset C_{2}$. Então $f$ se estende de modo holomorfo a uma função $g$ de $G \cup C_{0} \cup S_{1}(G)$ satisfazendo $g(w)=f(w), w \in G \cup C_{0}$ e $g(w)=S_{2}\left(f\left(S_{1}(w)\right)\right), w \in S_{1}(G)$.

Demonstração: Ver [3].

A seguir apresentamos alguns resultados sobre curvas em superfícies mínimas que fazem uso da superfície conjugada em sua demonstração. Suas provas podem ser encontradas em [18].

Proposição 1.5.3. Sejam $S=f(M)$ uma superfície mínima do $\mathbb{R}^{3}$ não planar com dados de Weierstrass $(g, d H)$, $\alpha$ uma geodésica em $M$ e $\beta:=f \circ \alpha$. Então

(a) $\{\beta\}$ é um segmento de reta se e só se $\left(\frac{d g}{g} d H\right)\left(\alpha^{\prime}\right) \in i \mathbb{R}$.

(b) $\{\beta\}$ está contida num plano se e só se $\left(\frac{d g}{g} d H\right)\left(\alpha^{\prime}\right) \in \mathbb{R}$.

Teorema 1.5.4 (Princípios de simetria de Schwarz para superfícies mínimas). Seja $S$ uma superficie minima em $\mathbb{R}^{3}$. Então 
(a) se $S$ contém um segmento de uma reta então $S$ é invariante por uma rotação de 180 graus ao redor dessa reta e,

(b) se $S$ é não planar e contém uma geodésica plana então $S$ é simétrica com relação ao plano que contém essa geodésica.

O teorema acima nos permite então construir superfícies mínimas obtendo apenas peças fundamentais da superfície que sejam delimitadas por geodésicas planas ou retas que ela contém e então estendemos essa peça para obter toda a superfície. 


\section{Capítulo 2}

\section{Obtendo novas superfícies a partir do catenóide}

Neste capítulo vamos obter equações algébricas para superfícies de Riemann $M$ que representem a geometria da superfície mínima obtida em termos das simetrias e das alças da superfície. Comparando as duas superfícies mínimas da figura 2.1 ( $M_{n}$-Costa-Hoffman-Meeks e "birdcage-catenóides") vemos que se compactificarmoas obtemos a mesma superfície de Riemann. O que muda são apenas os tipos de fins e a sua posição no $\mathbb{R}^{3}$. Isto nos leva a crer na existência de alguma relação entre seus dados de Weierstrass. O exemplos e a teoria aqui apresentados estão baseados em [16]. O mesmo autor descreve uma técnica para a troca de alças por fins planares em $[17]$.

Estudaremos nesse capítulo o caso em que a superfície tem dois fins catenoidais. Dos teoremas de López-Ros (1.4.17) e Schöen (1.4.18) sabemos que a única superfície mínima completa com curvatura total finita mergulhada de gênero zero com dois fins catenoidais é o catenóide. Logo, outra superfície com dois fins catenoidais tem que ter gênero positivo e deixará de ser mergulhada. Veremos então como se modificam os dados de Weierstrass em relação aos do catenóide quando adicionamos diversos tipos de alças ao catenóide. Veremos ainda, no capítulo seguinte, que em alguns casos é possível, com uma ligeira alteração na equação algébrica de $M$, trocar alças por fins catenoidais.

\subsection{Catenóide}

Compactificando o catenóide de acordo com a figura 2.3 obtemos a esfera $\widehat{\mathbb{C}}$ (identificando os fins do catenóide com os pólos norte e sul de $\widehat{\mathbb{C}})$. Assim $M=\widehat{\mathbb{C}} \backslash\{0, \infty\}$. 

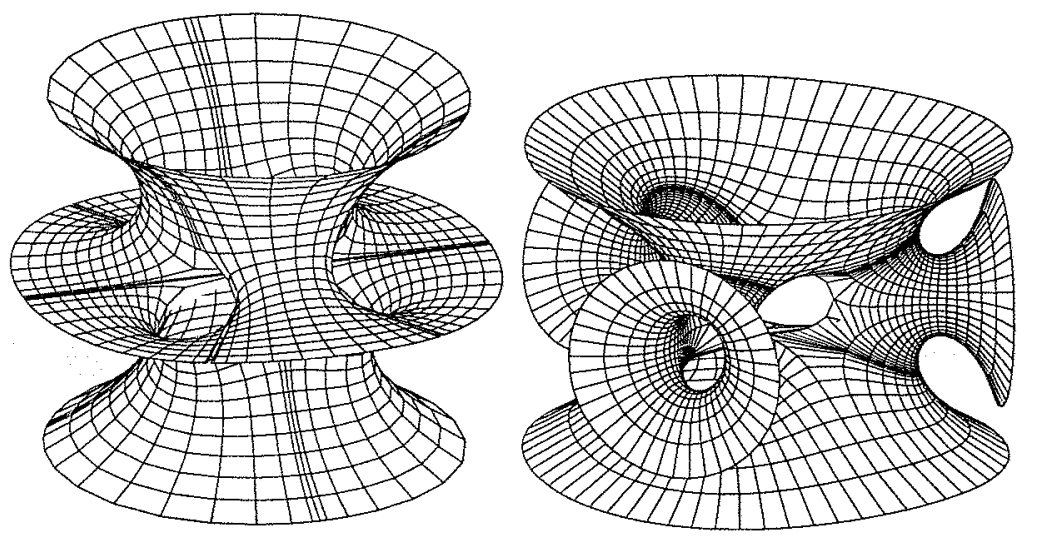

Figura 2.1: $M_{3}$-Costa-Hoffman-Meeks e o "birdcage-catenóide" de gênero 2.

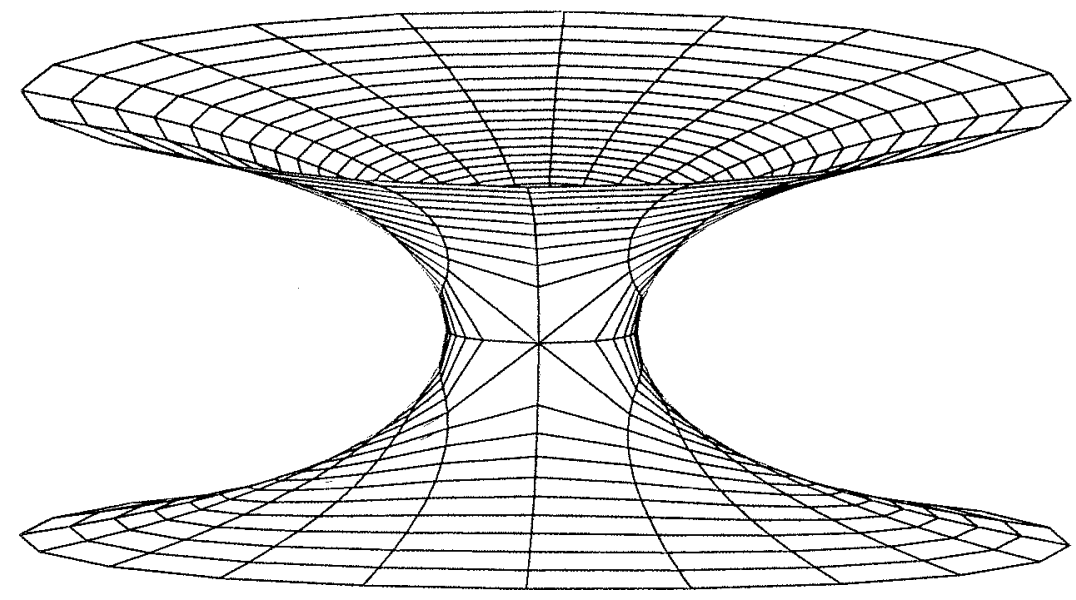

Figura 2.2: O catenóide.
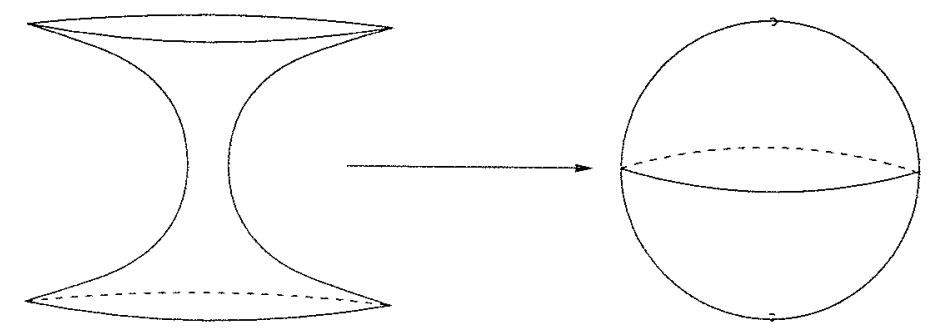

Figura 2.3: Compactificação do catenóide. 
Para encontrar os dados de Weierstrass do catenóide vamos considerar o seu vetor normal, lembrando que $N=$ stog. Isto nos dá $g(0)=\infty$ e $g(\infty)=0$. Como a imagem de $N$ restrita à cintura do catenóide está contida no equador de $\widehat{\mathbb{C}}$ temos que $g \subset S^{1}$, isto é, $|g|=1$ ao longo dessa curva. Em particular temos $g(1)=1$. Como $g \in \mathcal{O}(\widehat{\mathbb{C}})$ e é injetora (pois $N$ o é) temos que $g$ é um biholomorfismo de $\widehat{\mathbb{C}}$ e portanto uma aplicação de Möbius. Com os três valores acima podemos determinar $g$ ao longo de $\widehat{\mathbb{C}}$ :

$$
g(z)=\frac{1}{z}
$$

Considere agora um biholomorfismo $h$ em $\widehat{\mathbb{C}}$ que corresponde a uma rotação de 90 graus em torno do eixo $x_{2}$, ie, $h(\infty)=1, h(1)=0$ e $h(0)=-1$. Disto temos $h(z)=\frac{z-1}{z+1}$ ou, equivalentemente, $z=\frac{1+h}{1-h}$ e portanto

$$
\dot{g}=\frac{1-h}{1+h}
$$

Para obtermos a diferencial $d H$ devemos lembrar que $\operatorname{deg}(d H)=-\chi(\bar{M})=-2$, ou seja, ela deve ter dois pólos a mais do que zeros. Mas o pólos de $d H$ devem estar nos fins da superfície e os zeros nos pontos onde $g+\frac{1}{g}=\infty$. Não existem tais pontos, logo $d H$ deve ter um pólo simples em cada fim, dando o crescimento logarítmico que caracteriza os fins catenoidais (neste caso o crescimento logarítmico é na terceira coordenada). Portanto

$$
d H=c \frac{d h}{(h-1)(h+1)}=c \frac{d h}{h^{2}-1} .
$$

A constante $c$ acima é real pois ao longo da cintura do catenóide a terceira coordenada da representação de Weierstrass deve se anular, ie, $d H \in i \mathbb{R}$. Ao longo da cintura temos que $g \in S^{1}$ donde $h \in i \mathbb{R} \Rightarrow h(t)=i t$ para um certo parâmetro $t \in \mathbb{R}$ e então

$$
c \frac{d h}{h^{2}-1}=c \frac{i d t}{-t^{2}-1} \in i \mathbb{R} \Longleftrightarrow c \in \mathbb{R}
$$

Podemos escolher $c=1$ pois esta constante aparece em todas as coordenadas e portanto representa uma dilatação ou contração uniforme nos três eixos e a equação das superfícies mínimas é invariante por dilatações.

De acordo com resultado citado em [16], os períodos em torno dos fins se anulam, pois qualquer curva em torno de cada um deles é homotópica a uma curva simétrica em relação aos planos $x_{2} x_{3}$ e $x_{1} x_{3}$ simultaneamente. A simetria com relação ao plano $x_{2} x_{3}$ implica que o vetor período está na direção de $x_{1}$ e a simetria relativa ao plano $x_{1} x_{3}$ faz com que o vetor período esteja na direção de $x_{2}$. 
Com o objetivo de parametrizar apenas uma fatia do catenóide e obter toda a superfície a partir das simetrias dadas pelas catenárias (isto será muito útil no momento em que adicionarmos alças ao catenóide) podemos considerar os seguintes dados de Weierstrass:

$$
g^{k}=\left(\frac{1-h}{1+h}\right)^{k_{0}} \text { e } d H=\frac{d h}{h^{2}-1} .
$$

Com isso $g$ e $h$ são funções holomorfas com pontos de ramificação na esfera de Riemann, ie, $g$ e $h$ são recobrimentos ramificados de $\widehat{\mathbb{C}}$. Os pontos de ramificação de $g$ são 0 e $\infty$ e os de $h$ são 1 e -1 com as seguintes multiplicidades:

$$
\begin{array}{c|c|c}
\widehat{\mathbb{C}} & \infty & 0 \\
\hline g & 0^{n_{0}} & \infty^{n_{0}} \\
\hline h & 1^{m_{0}} & -1^{m_{0}}
\end{array} \text { com } n_{0}=\frac{k_{0}}{\left(k_{0}, k\right)} \text { e } m_{0}=\frac{k}{\left(k_{0}, k\right)}
$$

Deste modo $d H$ continua tendo ordem -1 em cada um dos fins pois ord $(d H)=$ $\left(m_{0}-1\right)-m_{0}=-1$ o que implica $\operatorname{deg}(d H)=-2$ (obtendo de fato uma superfície de Riemann homeomorfa à esfera.)

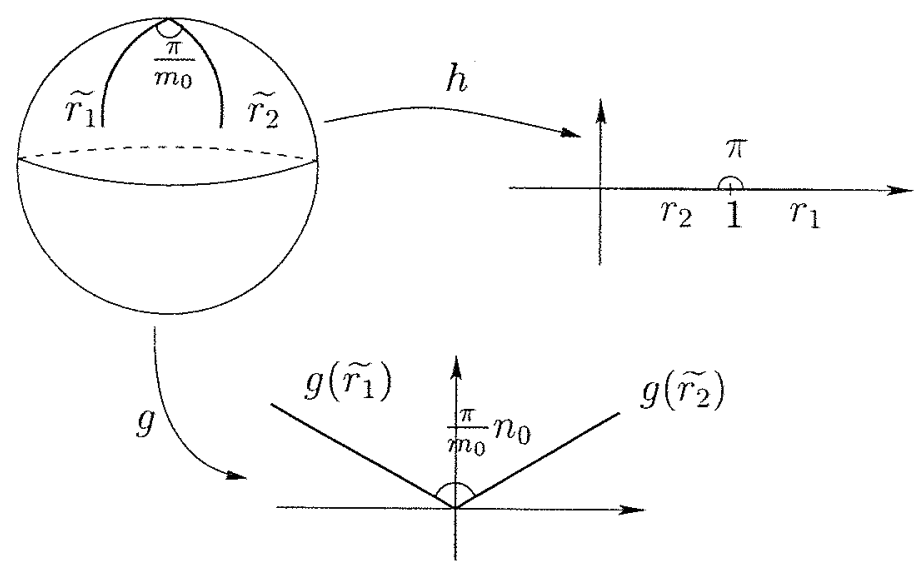

Figura 2.4: Fatias do catenóide.

Dados $k$ e $k_{0}$ em (2.2) podemos determinar a largura da fatia de catenóide obtida, ie, o ângulo entre os planos de simetria que a delimitam. Para isso, consideremos a pré-imagem em $\widehat{\mathbb{C}}$ por $h$ do eixo real. Esta pré-imagem é levada em uma geodésica plana do catenoíde, pois $h \in \mathbb{R}$ implica $\frac{d g}{g} d H \in \mathbb{R}$. Sejam então $r_{1}$ e $r_{2}$ arcos de $\widehat{\mathbb{C}}$ partindo de um dos fins $(h=1$ ou $h=-1)$ do catenóide com imagem real por $h$. Como a ordem de $h$ nos fins é $m_{0}$ então o ângulo entre as pré-imagens de $r_{1}$ e $r_{2}$, $\widetilde{r_{1}}$ e $\widetilde{r_{2}}$ respectivamente, em $\widehat{\mathbb{C}} \dot{e} \frac{\pi}{m_{0}}$. Agora, com relação a $g$, o ângulo entre $g\left(\widetilde{r_{1}}\right)$ e 
$g\left(\widetilde{r_{2}}\right)$ é $\frac{\pi}{m_{0}} n_{0}$, pois $g$ tem ordem $n_{0}$ nos fins, como pode ser visto na figura 2.4 . Se $\frac{\pi}{l_{0}}$ é o ângulo entre os planos que contêm as imagens de $\widetilde{r_{1}}$ e $\widetilde{r_{2}}$ pela representação de Weierstrass temos

$$
\frac{\pi}{l_{0}}=\frac{\pi}{m_{0}} n_{0} \Rightarrow l_{0}=\frac{m_{0}}{n_{0}}=\frac{k}{k_{0}}
$$

e isso nos dá o ângulo entre os vetores normais que é igual ao dos planos de simetria.

Exemplos:

1. Se quisermos um quarto do catenóide, (ie, ângulo $\frac{\pi}{l_{0}}=\frac{\pi}{2}$ ) devemos ter $l_{0}=2$, ou seja, $k=2 k_{0}$.

2. Se $k_{0}=2 k$ temos $l_{0}=2 \pi$ o que dá um ângulo entre os planos de simetria de $2 \pi$, cobrindo o catenóide duas vezes.

No que se segue vamos apenas considerar parametrizações do catenóide sem pontos de ramificação para $g$ no fins. Isto quer dizer que $n_{0}=1 \Rightarrow k_{0} \mid k$. Vamos também manter a simetria equatorial para facilitar a resolução do problema de alguns períodos.

\subsection{Alças em uma superfície mínima}

Existem diversos tipos de alças que podem ser adicionados a uma superfície mínima. Ao fazermos isso aumentamos o gênero da superfície de Riemann que gera a superfície mínima no $\mathbb{R}^{3}$, o que dificulta em geral a resolução do problema dos períodos. Vamos aqui adicionar alças à superfície que representem algum tipo de simetria reflexiva para ela, isto é, as alças em questão conterão geodésicas planas e portanto os planos que as contêm serão planos de simetria para a superfície no $\mathbb{R}^{3}$. Vamos supor ainda que nessas alças o vetor normal à superfície assume 0 e $\infty$ em dois pontos das geodésicas planas contidas nessas alças (isto faz com que os planos de simetria sejam perpendiculares ao plano $x_{1} x_{2}$ ).

As alças são aqui classificadas de acordo com o ângulo entre os planos de simetria nelas contidos.

Definição 2.2.1. Dizemos que uma alça é uma $l$-alça, $l \in \mathbb{N}^{*}$, se os seus planos de simetria formam um ângulo de $\frac{2 \pi}{l}$.

Em particular, 2-alças são chamadas alças de Schwarz e 4-alças são chamadas alças de Neovius. 


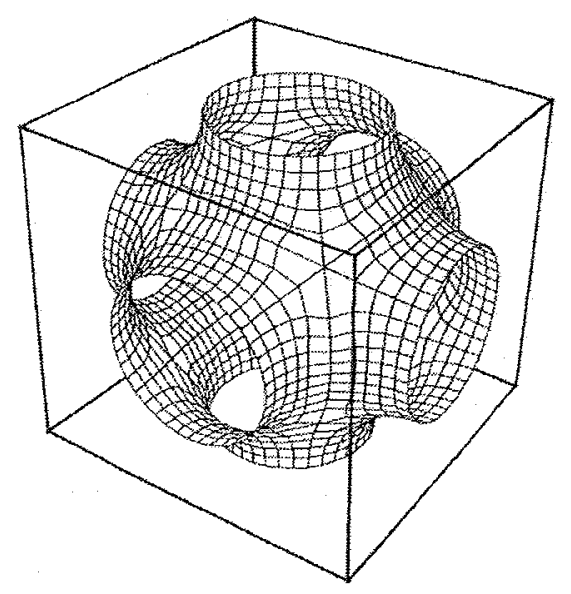

Figura 2.5: Alças de Schwarz numa superfície mínima.

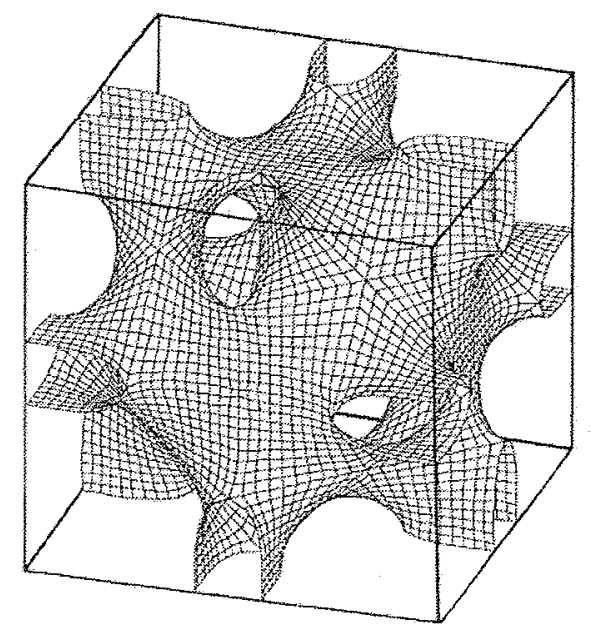

Figura 2.6: Alças de Neovius numa superfície mínima. 
As figuras 2.5 e 2.6 mostram alças do tipo de Schwarz e de Neovius respectivamente.

Nesta seção vamos obter exemplos de superfícies mínimas puxando e empurrando alças no catenóide com planos de simetria nos seus bordos e vamos manter a simetria na superfície com relação ao plano que contém a cintura do catenóide.

Como um primeiro exemplo vamos construir uma superfície cuja existência foi provada por Hoffman e Karcher em 1986 e é citada [10]. Essa superfície tem gênero 1 e é períodica, tendo sua peça fundamental parecida com um catenóide que tem duas alças de Schwarz saindo dele. Em cada alça há um plano de simetria e a superfície é continuada por reflexão nesses planos. Por esse motivo essa superfície é chamada de cerca de catenóides. Com um método semelhante Wohlgemuth construiu uma superfície mínima duplamente periódica de gênero $2 \mathrm{com}$ dois fins catenoidais e quatro alças de Schwarz.

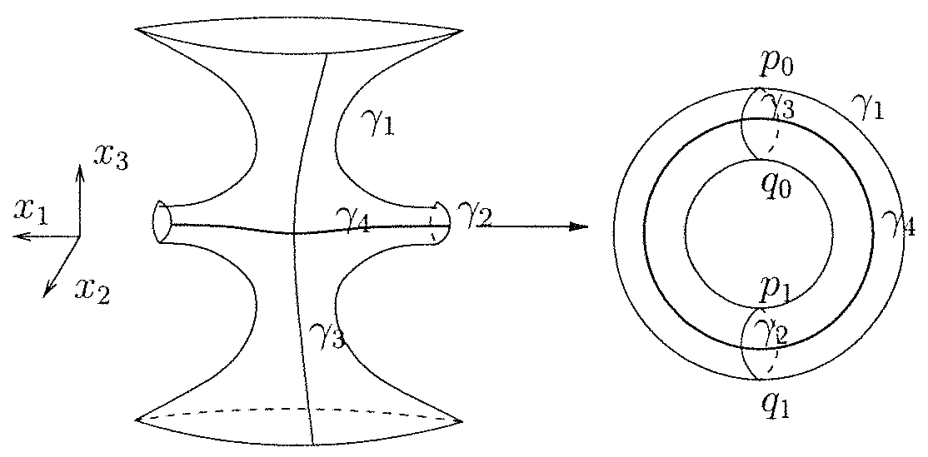

Figura 2.7: Compatificação da cerca de catenóides.
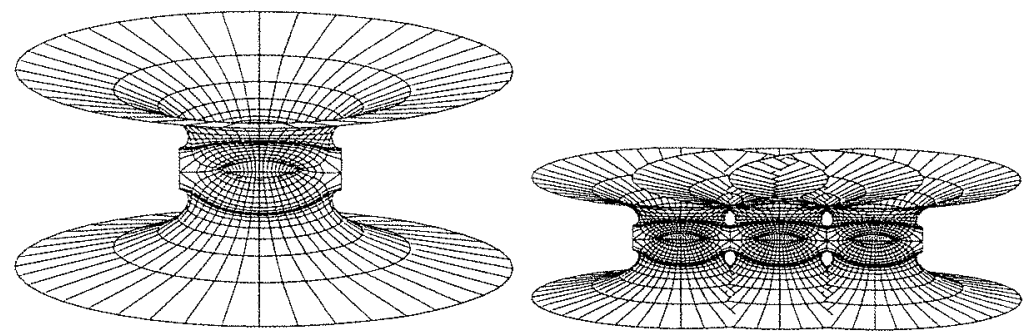

Figura 2.8: A peça fundamental e as reflexões da cerca de catenóides.

Podemos obter os dados de Weierstrass para a cerca de catenóides a partir da geometria da superfície usando funções elípticas, mas seguiremos o tipo de construção usado para o catenóide da seção anterior. Para isto observemos na figura 2.7 que a superfície de Riemann em questão é um toro $T$ retangular, pois este possui uma involução anti-holomorfa, a saber a reflexão com relação ao plano que contém a curva 
que liga $p_{0} p_{1}$, que tem um conjunto de pontos constituído por duas componentes conexas.

Considere agora a involução $\rho: T \rightarrow T$ que corresponde à rotação de $180^{\circ}$ do toro em relação ao eixo $x_{3}$ no $\mathbb{R}^{3}$. Esta involução tem ordem 2 , ou seja, $\rho^{2}=i d$ e 4 pontos fixos, a saber $p_{0}, p_{1}, q_{0}$ e $q_{1}$, e portanto de 1.3 .32 temos $\chi(T / \rho)=\frac{\chi(T)}{2}+4 \frac{(2-1)}{2}=2$.

Assim $T / \rho$ é biholomorfo à esfera $\widehat{\mathbb{C}}$. Qualquer biholormorfismo de $\widehat{\mathbb{C}}$ em $\widehat{\mathbb{C}}$ é uma aplicação de Möbius que pode ser determinada por 3 valores. Seja então $\tilde{h}: T /$ $\rho \rightarrow \widehat{\mathbb{C}}$ satisfazendo $\tilde{h}\left(\left[p_{0}\right]\right)=1, \tilde{h}\left(\left[q_{0}\right]\right)=-1$ e $\tilde{h}\left(\left[p_{1}\right]\right)=a, a \in(-1,0)$. A involução no toro dada pela reflexão relativamente à curva indicada na Figura 2.7 induz uma involução anti-holomorfa em $\hat{\mathbb{C}}$ que troca 1 e -1 e mantém 0 fixo, ou seja, $z \rightarrow-\bar{z}$, que corresponde a uma reflexão relativa ao eixo imaginário. Como os pontos $p_{1}$ e $q_{1}$ são simétricos com relação a essa involução concluímos que $h\left(\left[q_{1}\right]\right)=-\overline{h\left[p_{1}\right]}=-a$. Temos então que $h: T \rightarrow \widehat{\mathbb{C}}$ definida por $h:=\tilde{h} \circ \rho$ é holomorfa de $T$ em $\widehat{\mathbb{C}}$ satisfazendo a tabela de valores e multiplicidades abaixo:

$$
\begin{array}{c|c|c|c|c}
T & p_{0} & q_{0} & p_{1} & q_{1} \\
\hline h & 1^{(2)} & -1^{(2)} & a^{(2)} & -a^{(2)}
\end{array} \text { com } a \in(-1,0) .
$$

Os valores entre parênteses acima indicam as multiplicidades das imagens. Neste caso a multiplicidade dos valores $a$ e $-a$ deve ser dois pois cada um dos pontos $p_{1}$ e $q_{1}$ é ponto fixo da involução $\rho$. Mas como $h$ é um recobrimento ramificado próprio de $\widehat{\mathbb{C}}$ (pois $h \in \mathcal{O}(T, \widehat{\mathbb{C}})$, com $T$ compacta) temos que todos os pontos devem ter a mesma multiplicidade.

$O$ vetor normal desta superfície aponta verticalmente para cima ou para baixo no fins e nos pontos correspondentes a $p_{1}$ e $q_{1}$. Assim, além do zero e do pólo simples nos fins catenoidais, deve ter um zero e um pólo simples no bordo de cada uma das alças, assim, o grau de $g$ aumenta de 1 em relação ao do catenóide, ie, $\operatorname{deg}(g)=2$. Disto obtemos a equação algébrica:

$$
g^{2}=\frac{1-h}{1+h} \frac{h-a}{h+a}
$$

Como $d H$ deve ter pólos somente nos fins e zeros onde $g+\frac{1}{g}=\infty$, obtemos

$$
d H=\frac{d h}{h^{2}-1} .
$$

De fato podemos ver na equação acima que os pólos de $d H$ são $h=1$ e $h=-1$ e $h$ tem ordem dois neste pontos, o que dá $\operatorname{ord}(d h)=1$ fazendo com que $d H$ tenha ordem -1, ou seja, pólos simples. Os zeros de $d H$ são obtidos quando $d h=0$, ie, nos 
pontos de ramificação de $h$ diferentes de 1 e -1 que são exatamente $a$ e -a. Estes zeros também são simples pois novamente $d h$ tem ordem 1 nesses pontos, assim como a ordem dos pólos de $g+\frac{1}{g}$.

Esses dados de Weierstrass induzem simetrias da superfície como verificamos a seguir.

Gostaríamos que o valor de $g$ na curva $\gamma_{1}$ indicada na superfície que vai do fim à alça (ie, a curva indicada na figura que liga $p_{0}$ a $q_{1}$ ) fosse real e no toro essa curva tem imagem real por $h$. Logo $h=t$, onde $-a<t<1$ e então $g^{2}=\frac{1-t}{1+t} \frac{t-a}{t+a}>0 \Rightarrow$ $g(t) \in \mathbb{R}$.

Para a curva $\gamma_{2}$ indicada no bordo da alça gostaríamos que $g$ fosse imaginária pura. Ao longo dessa curva $h$ é real e varia entre $a$ e $-a$. Então $g^{2}=\frac{1-t}{1+t} \frac{t-a}{t+a}<0$ e portanto $g \in i \mathbb{R}$ ao longo dessa curva.

Para a curva $\gamma_{3}$ indicada que liga um fim ao outro, isto é, $p_{0}$ a $p_{1}$, gostaríamos que $g$ também fosse imaginária pura. Para determinar os valores de $h$ ao longo dessa curva basta observar que ela é perpendicular à curva que liga $p_{0}$ a $q_{1}$ em $p_{0}$ e que este ponto é um ponto de ramificação de ordem 1 e portanto $h$ sobre esta curva é real, isto é, $h \in \mathbb{R}, h \in[1, \infty] \cup[-\infty,-1]$, donde $g^{2}=\frac{1-h}{1+h} \frac{h-a}{h+a}<0$ implicando $g \in i \mathbb{R}$.

Para a curva $\gamma_{4}$ indicada que liga uma alça à outra gostaríamos que $g \in S^{1}$. A fim de determinar o valor de $h$ ao longo desta curva basta observar a involução $\widetilde{I}$ no toro dada pelo rebatimento ao longo dela. Esta involução induz em $\hat{\mathbb{C}}$ uma única involução $I$ (ie, uma aplicação de Möbius ou uma conjugada) que satisfaz $I(a)=-a, I(1)=-1$ e $I(-a)=a$ que implica $I(z)=-\bar{z}$, para $z \in \hat{\mathbb{C}}$. Como a curva em questão é fixa por $\tilde{I}$ então sua imagem é fixa por $I$ e então $h$ sobre essa curva é imaginária pura. Portanto $g^{2}=\frac{1-i t}{1+i t} \frac{i t-a}{i t+a}$. Mas

$$
\left\{\frac{1-t}{1+t}, \frac{t-a}{t+a}\right\} \subset S^{1}, \forall t \in \mathbb{R} \Rightarrow g^{2} \in S^{1} \Rightarrow g \in S^{1} .
$$

Com isso vamos mostrar que a superfície obtida tem todas as simetrias desejadas, ou seja, todas as curvas citadas acima são geodésicas planas. As curvas a que nos referimos abaixo são as mesmas da figura 2.7 .

Para uma das curvas que liga $p_{0}$ a $q_{1}$, digamos $\gamma_{1}$, (basta fazer uma pois a outra é

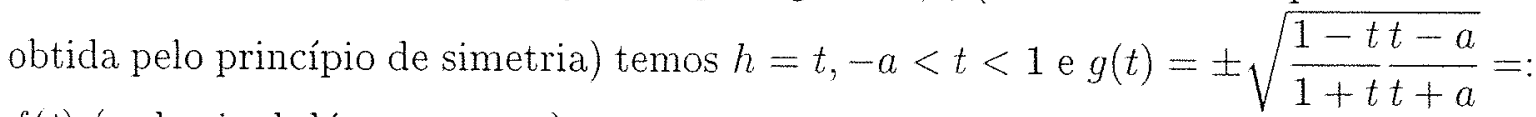
$f(t)$ (cada sinal dá uma curva), $-a<t<1$ e então

$$
\frac{d g}{g} d H\left(\gamma_{1}^{\prime}\right)=\frac{f^{\prime}(t)}{f(t)} d t \frac{d t}{t^{2}-1}\left(\gamma_{1}^{\prime}\right)=\frac{f^{\prime}(t)}{f(t)\left(t^{2}-1\right)} d t^{2}\left(\gamma_{1}^{\prime}\right) \in \mathbb{R}
$$


Para a curva $\gamma_{2}$ no bordo de uma das alças temos $h=t, a<t<-a$ e $g(t)=$ if $(t), a<t<-a$ para alguma $f$ real e portanto

$$
\frac{d g}{g} d H\left(\gamma_{2}^{\prime}\right)=\frac{i f^{\prime}(t)}{i f(t)} d t \frac{d t}{t^{2}-1}\left(\gamma_{2}^{\prime}\right)=\frac{f^{\prime}(t)}{f(t)\left(t^{2}-1\right)} d t^{2}\left(\gamma_{2}^{\prime}\right) \in \mathbb{R}
$$

Para a curva $\gamma_{3}$ que liga um fim ao outro temos $h=t,-1<t<1$ e $g(t)=$ if $(t),-1<t<1$ para alguma $f$ real. Portanto,

$$
\frac{d g}{g} d H\left(\gamma_{3}^{\prime}\right)=\frac{i f^{\prime}(t)}{i f(t)} d t \frac{d t}{t^{2}-1}\left(\gamma_{3}^{\prime}\right)=\frac{f^{\prime}(t)}{f(t)\left(t^{2}-1\right)} d t^{2}\left(\gamma_{3}^{\prime}\right) \in \mathbb{R} .
$$

Para a curva que liga as alças, $\gamma_{4}$, temos $h=i t$ e $g \in S^{1}$, ou seja, $g=e^{i f(t)}, t \in$ $I \subset \mathbb{R}$ para alguma $f$ real e então

$$
\frac{d g}{g} d H\left(\gamma_{4}^{\prime}\right)=\frac{i f^{\prime}(t) e^{i f(t)}}{e^{i f(t)}} d t \frac{i d t}{-t^{2}-1}\left(\gamma_{4}^{\prime}\right)=\frac{f^{\prime}(t)}{\left(t^{2}+1\right)} d t^{2}\left(\gamma_{4}^{\prime}\right) \in \mathbb{R} .
$$

Os períodos se anulam nos fins e nas alças pois eles têm dois planos de simetria perpendiculares $\left(x_{1} x_{3}\right.$ e $x_{2} x_{3}$ no caso dos fins e $x_{1} x_{2}$ e $x_{2} x_{3}$ no caso dos alças). Na curva onde $h=i t, t \in \mathbb{R}$ resta um período na direção do eixo $x_{1}$, dado pela reflexão nos planos de simetria das alças.

Definição 2.2.2. Sejam $m, n \in \mathbb{Z}$. Definimos $(m, n)$ como sendo o máximo divisor comum entre $m$ e $n$.

Comparando as equações (2.2) e (2.3) vemos que o lado direito da última é exatamente o lado direito da primeira multiplicado por um fator que nos dá as alças. Isto nos leva a considerar a seguinte equação

$$
g^{k}=\left(\frac{1-h}{1+h}\right)^{k_{0}}\left(\frac{h-a_{1}}{h+a_{1}}\right)^{k_{1}} \ldots\left(\frac{h-a_{N}}{h+a_{N}}\right)^{k_{N}}
$$

Esta equação define uma superfície de Riemann $M$ e funções $g$ e $h \in \mathcal{O}(M, \hat{\mathbb{C}})$.

Nestas condições, temos a seguinte tabela de multiplicidades para $g$ e $h$ :

$$
\begin{array}{c|c|c}
M & p_{j} & q_{j} \\
\hline g & 0^{n_{j}} & \infty^{n_{j}} \\
\hline h & a_{j}^{m_{j}} & -a_{j}^{m_{j}}
\end{array} \text { com } n_{j}=\frac{k_{j}}{\left(k, k_{j}\right)} \text { e } m_{j}=\frac{k}{\left(k, k_{j}\right)}, 0 \leq j \leq N .
$$

onde $p_{0}$ e $q_{0}$ são tais que $h\left(p_{0}\right)=a_{0}=1$ e $h\left(q_{0}\right)=-a_{0}=-1$ e portanto $g\left(p_{0}\right)=0$ e $g\left(q_{0}\right)=\infty$. Se considerarmos $g$ como a normal de Gauss e usarmos $h$ para construir a diferencial $d H=\frac{d h}{h^{2}-1}$ podemos provar o seguinte resultado: 
Teorema 2.2.3. Os dados de Weierstrass para uma $l_{0}$-fatia do catenóide com $N-1$ pares de $l_{j}$-alças, $j \in\{2,3,4, \ldots\}$ podem ser dados por

$$
g^{k}=\left(\frac{1-h}{1+h}\right)^{k_{0}}\left(\frac{h-a_{1}}{h+a_{1}}\right)^{k_{1}} \ldots\left(\frac{h-a_{N}}{h+a_{N}}\right)^{k_{N}} \text { e } d H=\frac{d h}{h^{2}-1}
$$

onde $k=m m c\left(l_{0}, l_{1}, \ldots, l_{N}\right)$. Então, com $k_{0}=\frac{k}{l_{0}} e k_{j}=k \frac{l_{j}-1}{l_{j}}$, todos os expoentes estão determinados. Os valores $a_{j} \in \mathbb{R}$ devem ser escolhidos de modo a obter o comportamento esperado dos perídos e eles também determinam o tamanho relativos das alças.

Demonstração: Para obtermos uma $l_{j}$-alça, os expoentes da equação (2.4) devem satisfazer:

1. A métrica deve ser regular (sem zeros e pólos) nos pontos $p_{j}$ e $q_{j}, j \geq 1$, ie

$$
0<\frac{|d H|}{|g|}\left(1+|g|^{2}\right)<\infty \Rightarrow 0<|d H|\left(\frac{1}{|g|}+|g|\right)<\infty
$$

Nos pontos $p_{j}$ e $q_{j}, j \geq 1$ a multiplicidade de $d H$ é $m_{j}-1$ e a de $g$ é $n_{j}$. Logo devemos ter

$$
m_{j}-1-n_{j}=0 \Rightarrow \frac{k}{\left(k, k_{j}\right)}-1-\frac{k_{j}}{\left(k, k_{j}\right)}=0 \Rightarrow k-k_{j}=\left(k, k_{j}\right) .
$$

2. Os planos de simetria de uma $l_{j}$-alça geram um ângulo de $\frac{2 \pi}{l_{j}}$. Considerando a aplicação normal esse ângulo orientado é de $2 \pi-\frac{2 \pi}{l_{j}}=2 \pi\left(\frac{l_{j}-1}{l_{j}}\right)$ como podemos ver na Figura 2.9. Como $h$ tem multiplicidade $m_{j}$ temos que $g$ leva ângulos de $\frac{2 \pi}{m_{j}}$ em $2 \pi\left(\frac{l_{j}-1}{l_{j}}\right)$. Lembrando que $g$ tem multiplicidade $n_{j}$ temos

$$
\frac{2 \pi}{m_{j}} n_{j}=2 \pi\left(\frac{l_{j}-1}{l_{j}}\right) \Rightarrow \frac{n_{j}}{m_{j}}=\frac{l_{j}-1}{l_{j}} \Rightarrow k_{j}=k\left(\frac{l_{j}-1}{l_{j}}\right) .
$$

Falta somente mostrar que a escolha de $k=m m c\left(l_{0}, l_{1}, \ldots, l_{N}\right)$ é compatível com as expressões acima. Mas 


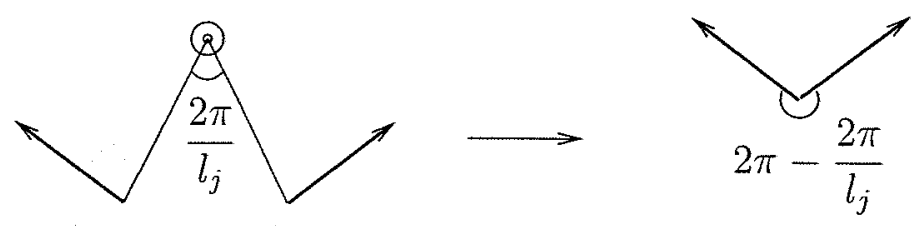

Figura 2.9: Ângulos do vetor normal em uma $l_{j}$-alça.

$$
\begin{aligned}
k-k_{j} & =k-k\left(\frac{l_{j}-1}{l_{j}}\right)=k-k+\frac{k}{l_{j}} \\
& =\frac{m m c\left(l_{0}, l_{1}, \ldots, l_{N}\right)}{l_{j}}=\frac{m m c\left(l_{0}, l_{1}, \ldots, l_{N}\right)}{l_{j}} \underbrace{\left(l_{j}, l_{j}-1\right)}_{=1} \\
& =\frac{k}{l_{j}}\left(l_{j}, l_{j}-1\right)=\left(k, k\left(\frac{l_{j}-1}{l_{j}}\right)\right) \\
& =\left(k, k_{j}\right) .
\end{aligned}
$$

\subsection{Exemplos}

Vamos construir alguns exemplos de superfícies mínimas de gênero 2, obtendo superfícies com diferentes tipos de simetria, apenas mudando o intervalo de variação dos $a_{k}$ no teorema 2.2.3.

\subsubsection{Catenóide duplamente periódico com quatro "alças de Schwarz"}

Para obtermos este tipo de superfície precisamos apenas de um quarto do catenóide, isto é, $l_{0}=2$ e duas alças de Schwarz: $l_{1}=2$ e $l_{2}=2$.

Destes valores e do teorema 2.2 .3 temos $k=2, k_{0}=1, k_{1}=1$ e $k_{2}=1$. Portanto

$$
g^{2}=\left(\frac{1-h}{1+h}\right)\left(\frac{h-a}{h+a}\right)\left(\frac{h-b}{h+b}\right) .
$$

Se escolhermos $a \in(-1,0)$ e $b \in(-\infty,-1)$ temos que as alças apontam para fora da superfície fazendo com que esta seja invariante por uma rotação de $180^{\circ} \mathrm{em}$ torno dos eixos $x$ e $y$, como na figura 2.11. Os valores de $a$ e $b$ também controlam o tamanho das alças.

As funções $g$ e $h$ têm a seguinte tabela de valores: 


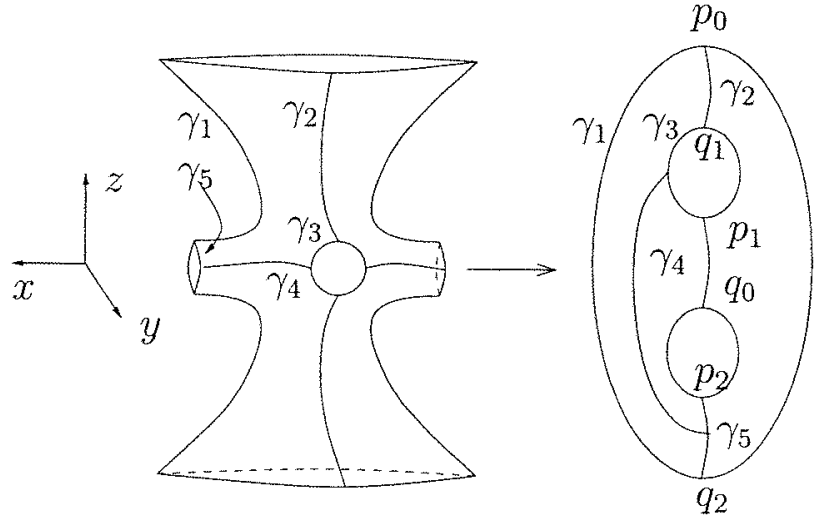

Figura 2.10: Compactificação do catenóide duplamente periódico.
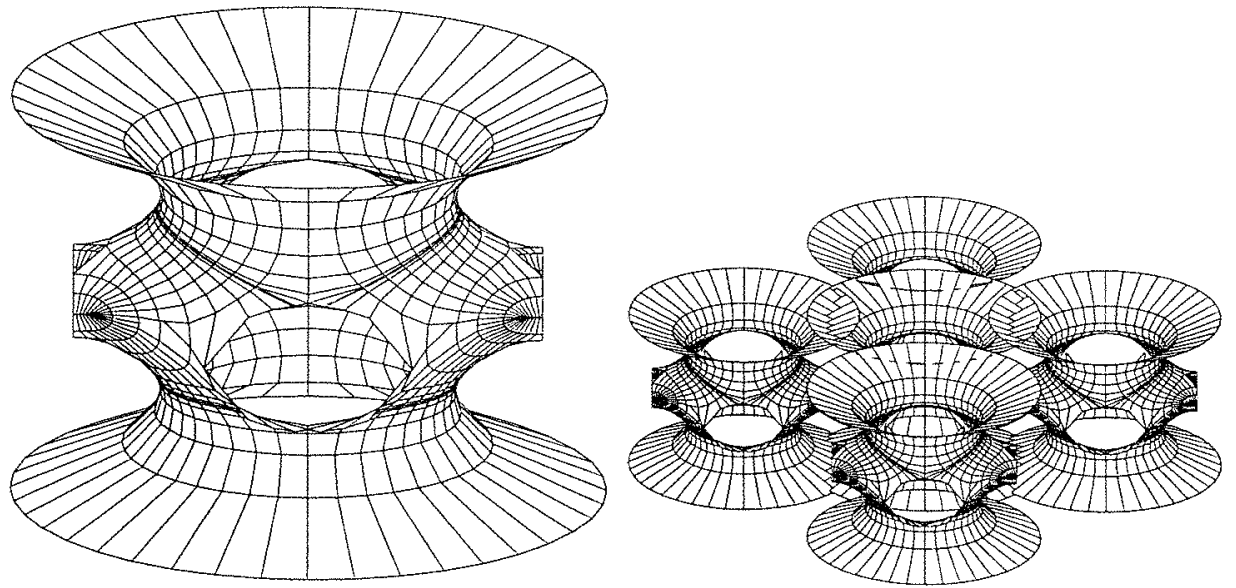

Figura 2.11: O catenóide duplamente periódico de gênero 2. 


\begin{tabular}{c|c|c|c|c|c|c}
$M$ & $p_{0}$ & $q_{0}$ & $p_{1}$ & $q_{1}$ & $p_{2}$ & $q_{2}$ \\
\hline$g$ & $0^{(0)}$ & $\infty^{(1)}$ & $0^{(1)}$ & $\infty^{(1)}$ & $0^{(1)}$ & $\infty^{(1)}$ \\
\hline$h$ & $1^{(2)}$ & $-1^{(2)}$ & $a^{(2)}$ & $-a^{(2)}$ & $b^{(2)}$ & $-b^{(2)}$
\end{tabular}

Observe que dependendo do modo como compactificamos a superfície mínima (sempre contraindo os fins a pontos) podemos obter superfícies de Riemann diferentes. Neste caso se identificamos os bordos das alças perpendiculares obtemos um bitoro. Se identificamos os bordos das alças paralelas obtemos uma nova superfície de Riemann chamada "birdcage", neste caso de gênero 3 . Veremos mais adiante que considerando essa superfície de Riemann podemos trocar as alças por fins catenoidais com uma ligeira alteração nos dados de Weierstrass obtidos abaixo. Com isto vemos que o gênero da superfície de Riemann não determina a superfície mínima em $\mathbb{R}^{3} \mathrm{e}$ que podemos obter uma mesma superfície mínima em $\mathbb{R}^{3}$ a partir de superfícies de Riemann distintas, ajustando para isso os dados de Weierstrass. Para este exemplo usaremos a compactificação da figura 2.10 .

As curvas indicadas na figura 2.10 geram as simetrias abaixo. Consideramos os extremos das curvas tais que o vetor normal sobre elas vale $0, \infty, \pm 1$ ou $\pm i$.

Se $\gamma_{1}$ é a curva que liga um fim à alça em torno do eixo $x_{1}$, a função $g$ deve ser real e, de fato, a pré-imagem de $\gamma_{1}$ em $M$ é uma curva $\widetilde{\gamma}_{1}$ (que identificaremos com $\gamma_{1}$, fazendo o mesmo para as outras curvas indicadas) tal que $h\left(\widetilde{\gamma_{1}}\right) \in(1,-b) \subset \mathbb{R}$. Assim temos $g^{2}=\frac{1-h}{1+h} \frac{h-a}{h+a} \frac{h-b}{h+b}>0$ o que implica $g \in \mathbb{R}$.

Se $\gamma_{2}$ é a curva sobre o bordo de uma das alças em torno do eixo $x_{1}$, a função $g$ deve ser imaginária pura, mas $\left\{\left.h\right|_{\gamma_{2}}\right\} \subset(-b, \infty) \cup(-\infty, b)$, pois $b$ e $-b$ são pontos de ramificação de ordem 1 e então $g^{2}=\frac{1-h}{1+h} \frac{h-a}{h+a} \frac{h-b}{h+b}<0$, ou seja, $g \in i \mathbb{R}$.

Se $\gamma_{3}$ é a curva que liga duas alças perpendiculares, então a função $g$ deve ser unitária, mas sobre $\gamma_{3}$ a função $h$ é imaginaria pura, logo $g^{2}=\frac{1-h}{1+h} \frac{h-a}{h+a} \frac{h-b}{h+b} \in S^{1}$ donde $g \in S^{1}$.

Se $\gamma_{4}$ é a curva sobre o bordo de uma das alças em torno do eixo $x_{2}$, então $g$ deve ser real. Mas $\left\{h_{\gamma_{4}}\right\} \in \mathbb{R}$ pois $\gamma_{3}$ e $\gamma_{4}$ são perpendiculares em $h=0$ que não é ponto de ramificação. Ainda temos que $h \in(a,-a)$, logo $g^{2}=\frac{1-h}{1+h} \frac{h-a}{h+a} \frac{h-b}{h+b}>0$, ou seja, $g \in \mathbb{R}$

Por fim, se $\gamma_{5}$ é a curva que liga o fim à alça em torno do eixo $x_{2}$, então $g$ deve ser imaginária pura. Observe que $\left.\left\{\left.h\right|_{\gamma_{5}}\right\} \subset(-a, 1)\right\}$. Consequentemente, $g^{2}=$ $\frac{1-h}{1+h} \frac{h-a}{h+a} \frac{h-b}{h+b}<0$, donde $g \in i \mathbb{R}$.

Vamos agora verificar que as curvas acima são geodésicas planas:

Ao longo de $\gamma_{1}$ temos $h(t)=t$, para algum parâmetro $t$, tal que $1<t<-b$ e 
$g=f(t)$ para alguma $f:[1,-b] \rightarrow \mathbb{R}$. Então

$$
\frac{d g}{g} d H\left(\gamma_{1}^{\prime}\right)=\frac{f^{\prime}(t)}{f(t)\left(t^{2}-1\right)} d t^{2}\left(\gamma_{1}^{\prime}\right) \in \mathbb{R} .
$$

Ao longo de $\gamma_{2}$ temos $h(t)=t, b<t<-b$ e $g(t)=i f(t)$ e então

$$
\frac{d g}{g} d H\left(\gamma_{2}^{\prime}\right)=\frac{i f^{\prime}(t)}{i f(t)\left(t^{2}-1\right)} d t^{2}\left(\gamma_{2}^{\prime}\right) \in \mathbb{R}
$$

Ao longo de $\gamma_{3}$ temos $h(t)=i t, t \leq 0$ e $g(t)=e^{i f(t)}$ e então

$$
\frac{d g}{g} d H\left(\gamma_{3}^{\prime}\right)=\frac{i f^{\prime}(t) e^{i f(t)}}{e^{i f(t)}\left(-t^{2}-1\right)} i d t^{2}\left(\gamma_{3}^{\prime}\right) \in \mathbb{R}
$$

Em $\gamma_{4}$ temos $h(t)=t, t \in(a,-a)$ e $g(t)=f(t) \log 0$

$$
\frac{d g}{g} d H\left(\gamma_{4}^{\prime}\right)=\frac{f^{\prime}(t)}{f(t)\left(t^{2}-1\right)} d t^{2}\left(\gamma_{4}^{\prime}\right) \in \mathbb{R} .
$$

Para $\gamma_{5}$ temos $h(t)=t,-a<t<1$ e $g(t)=-i f(t)$. Portanto,

$$
\frac{d g}{g} d H\left(\gamma_{5}^{\prime}\right)=\frac{i f^{\prime}(t)}{i f(t)\left(t^{2}-1\right)} d t^{2}\left(\gamma_{5}^{\prime}\right) \in \mathbb{R}
$$

Todos os períodos nos fins e nas alças se anulam pois existem dois planos de simetria perpendiculares em cada um deles.

\subsubsection{Catenóide simplesmente periódico com quatro "alças de Schwarz"}

Neste exemplo vamos puxar alças de Schwarz do catenóide, uma sobre a outra, obtendo uma superfície simplesmente periódica como na figura 2.13. Para isso escolhemos $a \in(-1,0)$. A outra alça vai crescer dentro da primeira, portanto $b \in(0,-a)$. Assim, a equação de $g$ tem a mesma forma de (2.5):

$$
g^{2}=\left(\frac{1-h}{1+h}\right)\left(\frac{h-a}{h+a}\right)\left(\frac{h-b}{h+b}\right) .
$$

A compactificação foi feita como na figura 2.12.

Vamos verificar as simetrias nas curvas:

A função $g$ deve ser real sobre $\gamma_{1}$. Mas $\left\{h\left(\gamma_{1}\right)\right\}=(-a, 1)$ o que implica $g^{2}=$ $\frac{1-h}{1+h} \frac{h-a}{h+a} \frac{h-b}{h+b}>0$ e portanto, $g=: f(t) \in \mathbb{R}$. Com isso segue-se que

$$
\frac{d g}{g} d H\left(\gamma_{1}^{\prime}\right)=\frac{f^{\prime}(t)}{f(t)\left(t^{2}-1\right)} d t^{2}\left(\gamma_{1}^{\prime}\right) \in \mathbb{R}
$$




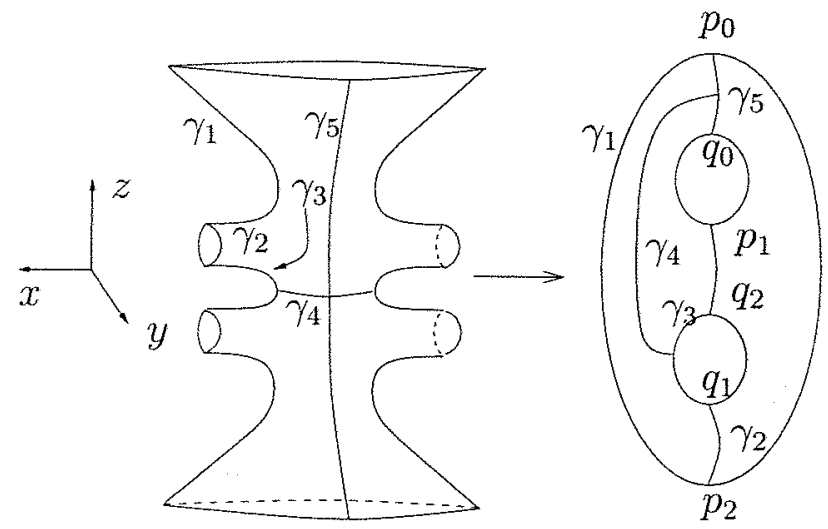

Figura 2.12: Compactificação da cerca de catenóides simplesmente periódica com 4 alças.

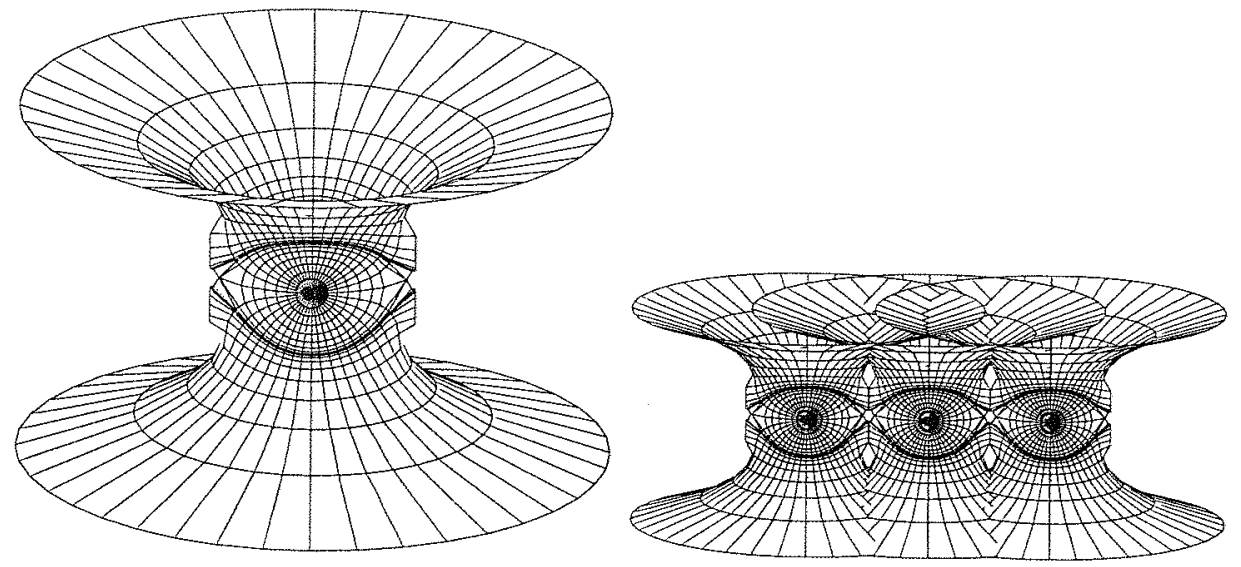

Figura 2.13: Cerca de catenóides simplesmente periódica com 4 alças. 
Sobre $\gamma_{2}$ a função $g$ deve ser imaginária pura, mas como $\left\{\left.h\right|_{\gamma_{2}}\right\}=(b,-a)$ temos que $g^{2}=\frac{1-h}{1+h} \frac{h-a}{h+a} \frac{h-b}{h+b}<0$. Logo $g \in i \mathbb{R}$ e então

$$
\frac{d g}{g} d H\left(\gamma_{2}^{\prime}\right)=\frac{i f^{\prime}(t)}{i f(t)\left(t^{2}-1\right)} d t^{2}\left(\gamma_{2}^{\prime}\right) \in \mathbb{R}
$$

Em $\gamma_{3}$, a função $g$ deve ser real e temos $\left\{h\left(\gamma_{3}\right)\right\}=(-b, b)$. Segue-se então que $g^{2}=\frac{1-h}{1+h} \frac{h-a}{h+a} \frac{h-b}{h+b}>0$ e portanto, $g \in \mathbb{R}$. Logo,

$$
\frac{d g}{g} d H\left(\gamma_{3}^{\prime}\right)=\frac{f^{\prime}(t)}{f(t)\left(t^{2}-1\right)} d t^{2}\left(\gamma_{3}^{\prime}\right) \in \mathbb{R}
$$

Para $\gamma_{4}$, a função $g$ deve ser unitária e ao longo de desta curva a função $h$ é imaginária pura pois $\gamma_{4}$ é perpendicular a $\gamma_{3}$ em $h=0$ que não é ponto de ramificação de $h$. Logo $g^{2}=\frac{1-h}{1+h} \frac{h-a}{h+a} \frac{h-b}{h+b} \in S^{1}$, donde $g \in S^{1}$ e então

$$
\frac{d g}{g} d H\left(\gamma_{4}^{\prime}\right)=\frac{i e^{i t} d t}{e^{i t}} \frac{i d t}{\left(-t^{2}-1\right)}\left(\gamma_{4}^{\prime}\right) \in \mathbb{R}
$$

Por fim, sobre $\gamma_{5}$ a função $g$ deve ser imaginária pura. Mas $\left\{\left.h\right|_{\gamma_{5}}\right\} \subset(1, \infty) \cup$ $(-\infty,-1)$ pois $\gamma_{5}$ é perpendicular a $\gamma_{4}$ em $h=1$ que é ponto de ramificação de ordem 1. Então $g^{2}=\frac{1-h}{1+h} \frac{h-a}{h+a} \frac{h-b}{h+b}<0$, ou seja, $g \in i \mathbb{R}$. Portanto,

$$
\frac{d g}{g} d H\left(\gamma_{5}^{\prime}\right)=\frac{i f^{\prime}(t)}{i f(t)\left(t^{2}-1\right)} d t^{2}\left(\gamma_{5}^{\prime}\right) \in \mathbb{R} .
$$

Com as simetrias dadas por estas geodésicas planas conseguimos mostrar apenas que os períodos nos fins se anulam e que os períodos nas alças estão na direção do eixo $y$, pois só há um plano de simetria vertical nas alças. Para obtermos uma superfície simplesmente periódica precisamos mostrar analiticamente que esses períodos se anulam, ie, mostrar que a segunda coordenada da representação de Weierstrass se anula numa curva fechada homotópica à curva no bordo de uma das alças (na outra o comportamento é o mesmo devido à simetria dada pela curva $\gamma_{5}$ ). Resumindo, precisamos mostrar que dado $a \in(-1,0)$ existe $b \in(0,-a)$ tal que

$$
\int_{\gamma} i\left(\frac{1}{g}+g\right) d H=0
$$

onde $g^{2}=\frac{1-h}{1+h} \frac{h-a}{h+a} \frac{h-b}{h+b}, d H=\frac{d h}{h^{2}-1}$ e $\gamma$ é uma curva fechada em $\hat{\mathbb{C}}$ com extremos $\gamma(0)=b$ e $\gamma(1)=-a$ satisfazendo $\{\gamma\} \subset\{z \in \mathbb{C}: 0 \leq \Re(z) \leq 1$ e $\Im(z) \geq$ $0\}$. 
Para cada $a \in(-1,0)$ fixado, definimos a função:

$$
f(b)=\int_{\gamma} \underbrace{\left(\frac{1}{g}+g\right) \frac{d h}{h^{2}-1}}_{\omega},
$$

onde

$$
g=\sqrt{\frac{1-h}{1+h} \frac{h-a}{h+a} \frac{h-b}{h+b}}
$$

Na região considerada para integração temos que $h \in(b,-a)$ implica $g \in i \mathbb{R}$ o que resulta que o integrando $\omega$ na equação $(2.7)$ é real e $h \in(0, b) \cup(-a, 1)$ implica $g \in \mathbb{R}$ e portanto $\omega \in i \mathbb{R}$. Com isso $\Re(f(b))$ não muda se estendermos os extremos de $\gamma$ para $\gamma(0)=0$ e $\gamma(1)=\frac{1-a}{2}$. Vamos então observar o sinal dos valores de $f(b)$ para $b=0$ e para $b=-a$ e tentar usar o teorema do valor intermediário.

Se $b=-a$ temos $g^{2}=\frac{1-h}{1+h}$, o que nos dá um quarto do catenóide e portanto $\Re(f(-a))=0$, pois não há alças, logo não há períodos reais.

Se $b=0$ temos $g^{2}=\frac{1-h}{1+h} \frac{h-a}{h+a}$ e portanto $g=\sqrt{\frac{1-h}{1+h} \frac{h-a}{h+a}}$. Cálculos diretos mostram que

$$
\frac{1}{g}+g=\frac{2 h(1+a)}{\sqrt{\left(1-h^{2}\right)\left(h^{2}-a^{2}\right)}} \text {. }
$$

Logo, podemos considerar, por homotopia, $\gamma(t)=t, 0<t<\frac{1-a}{2}$ e então temos

$$
f(0)=\int_{0}^{\frac{1-a}{2}} i \frac{2 t(1+a)}{\sqrt{\left(1-t^{2}\right)\left(t^{2}-a^{2}\right)}} \frac{d t}{t^{2}-1}
$$

onde todos os fatores do integrando são positivos à exceção de $t^{2}-1$ que é sempre negativo no intervalo considerado e $t^{2}-a^{2}=(t+a)(t-a)$ que é positivo se $t>-a$ e negativo se $t<-a$. Quando $t^{2}-a^{2}>0$ temos que o integrando é imaginário puro e portanto $\Re(f(0))=0$. Quando $t^{2}-a^{2}<0$ temos que o integrando é real negativo, donde $\Re(f(0))<0$.

Agora, de (2.7) temos

$$
\frac{d f}{d b}=\frac{d}{d b} \int_{\gamma} i\left(\frac{1}{g}+g\right) \frac{d h}{h^{2}-1}=i \int_{\gamma} \frac{d}{d b}\left(\frac{1}{g}+g\right) \frac{d h}{h^{2}-1}
$$

Definindo $g_{b}:=\frac{\partial g}{\partial b}$ e $K:=\sqrt{\frac{1-h}{1+h} \frac{h-a}{h+a}}$ temos 


$$
\begin{aligned}
g & =K \sqrt{\frac{h-b}{h+b}} \\
g_{b} & =K \frac{\frac{-(h+b)-(h-b)}{(h+b)^{2}}}{2 \sqrt{\frac{h-b}{h+b}}}=\frac{K(-h)}{\sqrt{\frac{h-b}{h+b}}(h+b)^{2}}
\end{aligned}
$$

Então

$$
\frac{\partial}{\partial b} \frac{1}{g}=\frac{-g_{b}}{g^{2}}=\frac{K h}{\sqrt{\frac{h-b}{h+b}}(h+b)^{2} K^{2} \frac{h-b}{h+b}}=\frac{h}{\sqrt{\frac{h-b}{h+b}}\left(h^{2}-b^{2}\right) K} .
$$

Disto vem que

$$
\begin{aligned}
g_{b}+\frac{1}{g_{b}} & =\frac{-K h}{\sqrt{\frac{h-b}{h+b}}(h+b)^{2}}+\frac{h}{\sqrt{\frac{h-b}{h+b}}\left(h^{2}-b^{2}\right) K} \\
& =\frac{h}{h^{2}-b^{2}}\left(-K \sqrt{\frac{h-b}{h+b}}+\frac{1}{K \sqrt{\frac{h-b}{h+b}}}\right) \\
& =\frac{h}{h^{2}-b^{2}}\left(-g+\frac{1}{g}\right) .
\end{aligned}
$$

Logo,

$$
f_{b}(b):=\frac{\partial f}{\partial b}(b)=i \int_{\gamma}\left(\frac{1}{g}-g\right) \frac{h}{h^{2}-b^{2}} \frac{d h}{h^{2}-1} .
$$

Se $b=-a$ temos $g=\sqrt{\frac{1-h}{1+h}}$ e então

$$
f_{b}(-a)=i \int_{\gamma}\left(\frac{1}{\sqrt{\frac{1-h}{1+h}}}-\sqrt{\frac{1-h}{1+h}}\right) \frac{h}{h^{2}-a^{2}} \frac{d h}{h^{2}-1}=i \int_{\gamma} \frac{2 h}{\sqrt{1-h^{2}}} \frac{h}{h^{2}-a^{2}} \frac{d h}{h^{2}-1} .
$$

A curva $\gamma$ acima é homotópica a um segmento de reta sobre o eixo real com um pequeno desvio no ponto $h=-a$. Mas como o integrando é imaginário puro para $h \in(0, b) \cup(-a, 1)$ temos que $\Re\left(f_{b}(-a)\right)$ é dada somente pela integral considerada sobre um pequeno semi-círculo em torno de $-a$.

Para calcular esta integral, observemos que $h$ é simétrica com relação ao eixo real, pois na superfície de Riemann temos uma involução que é o rebatimento com relação à curva $\gamma_{1}$. Esta involução induz uma involução anti-holomorfa em $\mathbb{C}$ que fixa o eixo real e portanto é a identidade. Assim, lembrando da orientação das curvas, temos

$$
-2 \int_{\gamma} \frac{2 h}{\sqrt{1-h^{2}}} \frac{h}{h^{2}-a^{2}} \frac{d h}{h^{2}-1}=\int_{\eta} \frac{2 h}{\sqrt{1-h^{2}}} \frac{h}{h^{2}-a^{2}} \frac{d h}{h^{2}-1}
$$


onde $\eta=\rho e^{i \theta}-a, 0 \leq \theta \leq 2 \pi$.

Mas como $h=-a$ é pólo simples do integrando, do teorema 1.3.28, temos que

$$
r e s_{-a}\left(\frac{2 h}{\sqrt{1-h^{2}}} \frac{h}{h^{2}-a^{2}} \frac{1}{h^{2}-1}\right)=\frac{1}{2 \pi i} \int_{\eta} \frac{2 h}{\sqrt{1-h^{2}}} \frac{h}{h-a} \frac{1}{h^{2}-1} \frac{1}{h+a} d h,
$$

Do comentário que segue a definição 1.3 .27 vem que

$$
\operatorname{res}_{-a}\left(\frac{2 h}{\sqrt{1-h^{2}}} \frac{h}{h^{2}-a^{2}} \frac{1}{h^{2}-1}\right)=\frac{2 a^{2}}{\sqrt{1-a^{2}}(-2 a)\left(a^{2}-1\right)} .
$$

Portanto, de (2.8), (2.9) e (2.10) temos

$$
\begin{aligned}
-2 \int_{\gamma} \frac{2 h}{\sqrt{1-h^{2}}} \frac{h}{h^{2}-a^{2}} \frac{d h}{h^{2}-1} & =2 \pi i \operatorname{res}_{-a}\left(\frac{2 h}{\sqrt{1-h^{2}}} \frac{h}{h^{2}-a^{2}} \frac{1}{h^{2}-1}\right) \\
& =\frac{2 \pi i 2 a^{2}}{\sqrt{1-a^{2}}(-2 a)\left(a^{2}-1\right)} .
\end{aligned}
$$

Donde

$$
\int_{\gamma} \frac{2 h}{\sqrt{1-h^{2}}} \frac{h}{h^{2}-a^{2}} \frac{d h}{h^{2}-1}=\frac{i \pi a}{\sqrt{1-a^{2}}\left(a^{2}-1\right)}
$$

Logo

$$
f_{b}(-a)=i \frac{i \pi a}{\sqrt{1-a^{2}}\left(a^{2}-1\right)}=\frac{-\pi a}{\sqrt{1-a^{2}}\left(a^{2}-1\right)}<0
$$

ie $\Re(f(0))<0, \Re(f(-a))=0$ e $\Re\left(f_{b}(-a)\right)<0$ e portanto existe $b_{0} \in(0,-a)$ tal que $\Re\left(f\left(b_{0}\right)\right)=0$, resolvendo assim este problema de período.

\subsubsection{Cerca de catenóides de gênero 2}

Podemos ainda construir um exemplo onde ao invés de puxarmos alças de Schwarz, como na seção anterior, empurraremos essas alças de modo a obter uma superfície como a de Hoffman-Karcher com um túnel. Não seria possível construir uma superfície mínima completa de curvatura total finita a partir do catenóide juntando duas alças de Schwarz obtendo um túnel no catenóide devido ao teorema de Schöen. Um esboço deste exemplo está na figura 2.14.

A compactificação dessa superfície mínima tem gênero dois como podemos ver na figura 2.15 .

Para a cerca de catenóides de gênero dois consideramos

$$
g^{2}=\frac{1-h}{1+h} \frac{h-a}{h+a} \frac{h-b}{h+b}, a \in(-\infty,-1), b \in(0,1) \text { e } d H=\frac{d h}{h^{2}-1}
$$




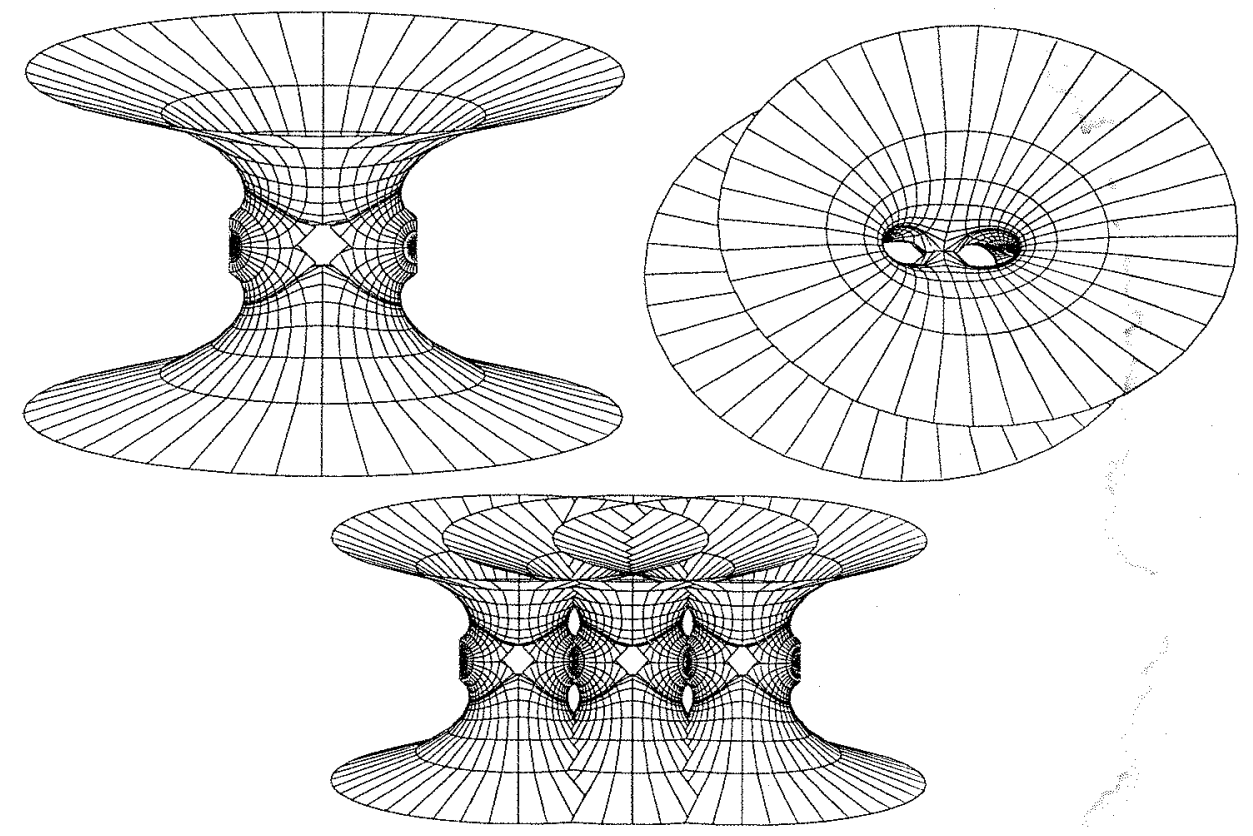

Figura 2.14: Cerca de catenóides de gênero 2 simplesmente periódico.

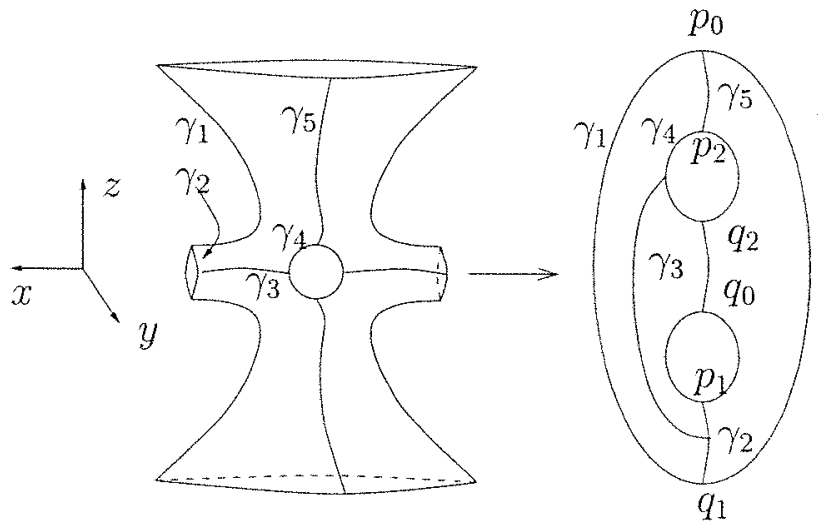

Figura 2.15: Compactificação da cerca de catenóides de gênero 2. 
Os dados de Weierstrass acima satisfazem de fato as simetrias requeridas para a superfície pois ao longo de $\gamma_{1}$ temos $h(t)=t, 1<t<-a$, donde $g \in \mathbb{R}$, e então

$$
\frac{d g}{g} d H\left(\gamma_{1}^{\prime}\right) \in \mathbb{R}
$$

Sobre $\gamma_{2}$ temos $h(t)=t, t \in[-a, \infty] \cup[\infty, a]$ e então $g \in i \mathbb{R}$, logo

$$
\frac{d g}{g} d H\left(\gamma_{2}^{\prime}\right) \in \mathbb{R}
$$

Em $\gamma_{3}$ temos $h(t) \in i \mathbb{R}$, pois é perpendicular a $\gamma_{2}$ num ponto regular e portanto $h(t)=i t$, para algum parâmetro real $t$ o que dá $g \in S^{1}$, resultando

$$
\frac{d g}{g} d H\left(\gamma_{3}^{\prime}\right) \in \mathbb{R}
$$

Para $\gamma_{4}$ temos $h(t)=t, 0<t<b, \operatorname{logo} g \in \mathbb{R}$. Assim

$$
\frac{d g}{g} d H\left(\gamma_{4}^{\prime}\right) \in \mathbb{R}
$$

Por fim, ao longo de $\gamma_{5}, h(t)=t$, o que implica $g \in i \mathbb{R}$, mostrando que

$$
\frac{d g}{g} d H\left(\gamma_{5}^{\prime}\right) \in \mathbb{R}
$$

Observamos que os fins são simétricos com relação aos planos $x_{1} x_{3}$ e $x_{2} x_{3}$, anulando portanto os períodos nos fins. As alças em torno do eixo $y$ são simétricas com relação aos planos $x_{1} x_{3}$ e $x_{1} x_{2}$, o que mostra que elas também não apresentam períodos reais. O período que não se anula por simetrias da superfície é o relativo a uma curva fechada homotópica à curva $\gamma_{2}+\gamma_{3}$ (considerando a parte dada pela reflexão com relação ao plano $x_{1} x_{3}$ ), onde $h \circ \gamma_{2}(t)=\frac{-a}{t}, 0<t<1$ e $h \circ \gamma_{3}=i t, 0<t<\infty$. Usando um método semelhante ao do exemplo anterior definimos

$$
f(b)=\int_{\gamma} i\left(g+\frac{1}{g}\right) d H,
$$

com $g^{2}$ e $d H$ dados por (2.11). Vamos aqui escolher o ramo positivo da raiz quadrada, como no exemplo anterior.

Se $b=0$ então,

$$
\begin{gathered}
g+\frac{1}{g}=\frac{2 h(1+a)}{\sqrt{\left(1-h^{2}\right)\left(h^{2}-a^{2}\right)}} . \\
f(0)=\int_{\gamma_{1}} i\left(\frac{2 h(1+a)}{\sqrt{\left(1-h^{2}\right)\left(h^{2}-a^{2}\right)}}\right) \frac{d h}{h^{2}-1}+\int_{\gamma_{2}}^{i}\left(\frac{2 h(1+a)}{\sqrt{\left(1-h^{2}\right)\left(h^{2}-a^{2}\right)}}\right) \frac{d h}{h^{2}-1} \\
=\int_{0}^{\infty} \frac{2 t(1+a)}{\sqrt{1+t^{2}} \sqrt{(t-a)(t+a)}} \frac{i d t}{t^{2}+1}+\int_{0}^{1} \frac{2 a^{3}(1+a)}{t \sqrt{a^{2}-t^{2}} \sqrt{1-t^{2}\left(t^{2}-a^{2}\right)} d t .}
\end{gathered}
$$


Lembrando que $a<-1$, concluímos que o integrando na segunda integral acima é negativo. Separando o intervalo de integração na primeira integral em $(0,-a)$ e $(-a, \infty)$, notamos que sobre o segundo intervalo a integral é imaginária pura e sobre o primeiro intevalo, após trocarmos $\sqrt{t^{2}-a^{2}}$ por $i \sqrt{a^{2}-t^{2}}$, a integral é real negativa. Logo $\Re f(0)<0$.

Se $b=1$ então $g^{2}=-\left(\frac{1-h}{1+h}\right)^{2}\left(\frac{h-a}{h+a}\right)$. A superfície é deformada de modo que a alça relativa ao valor $b$ desaparece e a superfície deixa de ser periódica, assemelhandose a dois catenóides unidos por um túnel (originalmente a alça relativa ao valor a). Esta superfície compactificada é uma esfera. A curva $\gamma$ agora corresponde a uma curva em torno do fim $h=1$. Então temos que $\gamma$ pode ser escolhida tal que $h(\gamma(t))=1+\rho e^{i t}, 0<t<2 \pi, \epsilon<|1+a|$. Então podemos escrever

$$
f(1)=\int_{\gamma} i\left(g+\frac{1}{g}\right) d H=\int_{\gamma} i g d H+\int_{\gamma} \frac{i}{g} d H .
$$

Mas

$$
\begin{aligned}
\int_{\gamma} i g d H & =\int_{\gamma}-\sqrt{\frac{h-a}{h+a}}\left(\frac{1-h}{1+h}\right) \frac{d h}{h^{2}-1} \\
& =\int_{\gamma} \sqrt{\frac{h-a}{h+a}} \frac{d h}{(h+1)^{2}} .
\end{aligned}
$$

O integrando acima é holomorfo no interior da curva $\gamma, \operatorname{logo}$

$$
\int_{\gamma} i g d H=0
$$

Porém, o integrando em

$$
\int_{\gamma} \frac{i}{g} d H=\int_{\gamma}-\sqrt{\frac{h+a}{h-a}} \frac{d h}{(h-1)^{2}}
$$

tem uma singularidade quando $h=1$. Então,

$$
-\int_{\gamma} \sqrt{\frac{h+a}{h-a}} \frac{d h}{(h-1)^{2}}=-2 \pi \operatorname{ires}_{1}\left(\sqrt{\frac{h+a}{h-a}} \frac{1}{(h-1)^{2}}\right) .
$$

Mas $h=1$ é um pólo duplo do integrando. Então

$$
\begin{aligned}
\operatorname{res}_{1}\left(\sqrt{\frac{h+a}{h-a}} \frac{1}{(h-1)^{2}}\right) & =\lim _{h \rightarrow 1} \frac{d}{d h}\left((h-1)^{2} \sqrt{\frac{h+a}{h-a}} \frac{1}{(h-1)^{2}}\right) \\
& =\lim _{h \rightarrow 1} \frac{d}{d h} \sqrt{\frac{h+a}{h-a}} \\
& =\frac{-a}{(1-a)^{\frac{3}{2}} \sqrt{1+a}} \in i \mathbb{R} .
\end{aligned}
$$


Logo

$$
\int_{\gamma}-\sqrt{\frac{h+a}{h-a}} \frac{d h}{(h-1)^{2}}=-2 \pi i\left(\frac{-a}{(1-a)^{\frac{3}{2}} \sqrt{1+a}}\right)=\frac{-2 \pi a}{(1-a)^{\frac{3}{2}} \sqrt{-1-a}}>0
$$

Observe que aqui, como a curva $\gamma$ completa uma volta num ponto de ramificação $(h=1)$, houve uma troca de ramo da raiz quadrada e portanto $\sqrt{1+a}=-i \sqrt{-1-a}$. Disto concluímos que $\Re f(1)>0$.

Portanto segue-se, do teorema do valor intermediário, que para todo $a \in(-\infty,-1)$ o problema de período para esta superfície tem solução. 


\section{Capítulo 3}

\section{Birdcage-catenóides}

Neste capítulo vamos mostrar a existência de uma família de superfícies mínimas em $\mathbb{R}^{3}$ chamadas "birdcage-catenóides" (ver [1]). Elas são imersões mínimas de superfícies de Riemann compactadas, chamadas de "birdcages", menos um número finito de pontos. Cada um desses pontos removidos dá origem a um fim catenoidal.

Esse tipo de superfície é baseado nos exemplos obtidos por D. Hoffman e J. Meeks, chamados $M_{n}$-Costa-Hoffman-Meeks, $n \geq 3$, que são superfícies do tipo da de Costa, ou seja, com os mesmos fins e simetrias, mas com gênero $n-1$. Na figura 3.1 temos um modelo para o "birdcage" de gênero 2 e a superfície $M_{3}$-Costa-Hoffman-Meeks. Vemos que se compactificarmos cada fim da superfície mínima obtemos o "birdcage". Esse processo pode ser efetuado para todo $n \geq 2$.
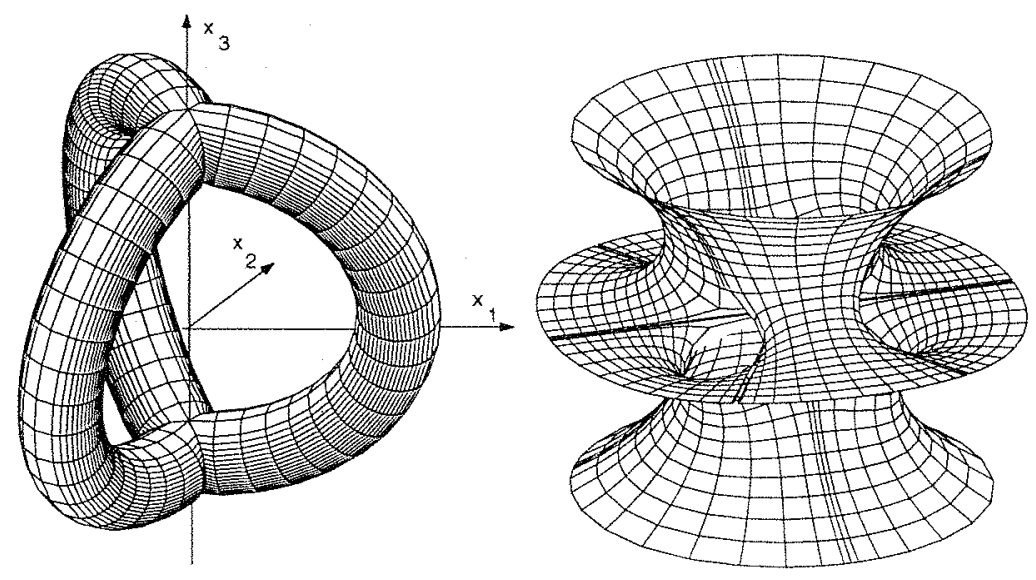

Figura 3.1: O "birdcage" de gênero 2 e a $M_{3}$-Costa-Hoffman-Meeks.

A superfície de Riemann compacta chama-se "birdcage" devido às suas simetrias, como podemos ver na figura 3.1. Se consideramos o "birdcage" de gênero $n-1$, 
notamos que ele tem um eixo de simetria rotacional ( $x_{3}$ no caso da figura) que deixa a superfície invariante por uma rotação de $\frac{360}{n}$ graus. Existem ainda $n$ planos verticais e um horizontal que deixam a superfície invariante por reflexão com relação a eles. Existe ainda uma simetria intrínseca que não pode ser visualizada no $\mathbb{R}^{3}$.

Queremos aqui mostrar o seguinte o resultado:

Teorema 3.0.1. Para cada inteiro $n \geq 2$ existe uma familia de superfícies mínimas completas em $\mathbb{R}^{3}$ tal que todo membro desta familia tem gênero $n-1$ e todos os seus fins são catenoidais, sendo dois verticais e $n$ horizontais.

A figura 3.2 representa um membro da família do enunciado do teorema quando $n=2$.

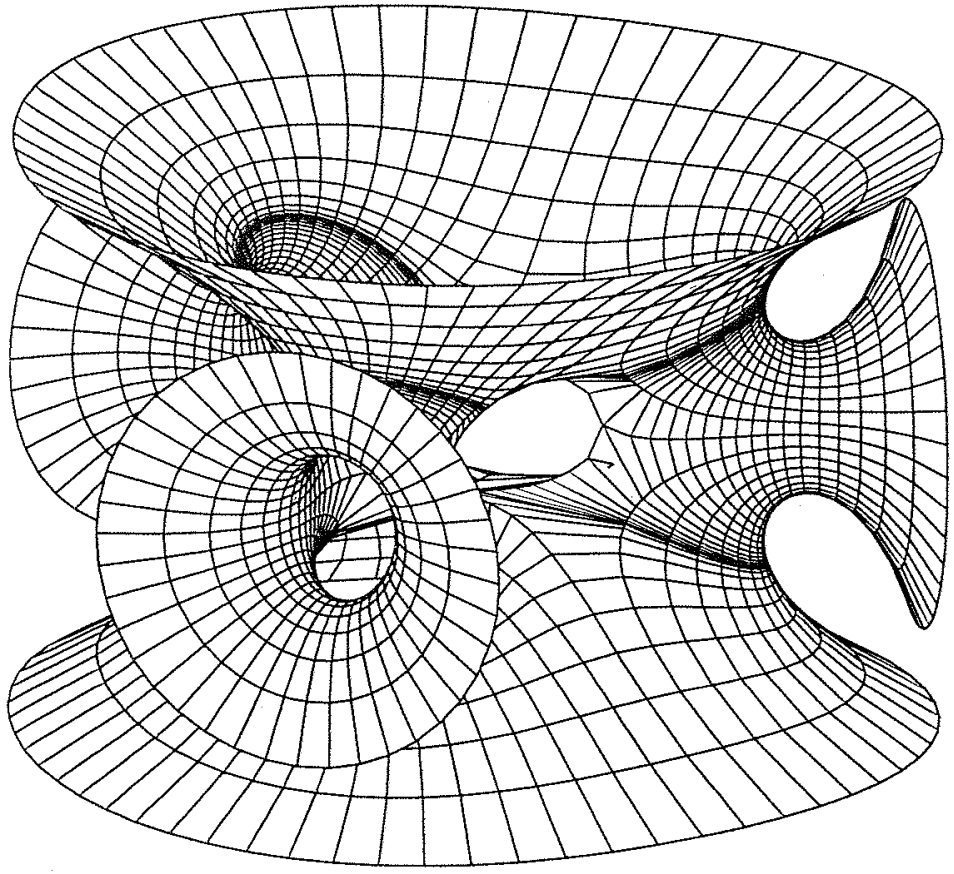

Figura 3.2: "Birdcage-catenóide" de gênero 2

Explorando as simetrias do "birdcage" e o comportamento esperado da superfície minima no $\mathbb{R}^{3}$ vamos obter os dados de Weierstrass que a representam. 


\subsection{Equação algébrica do "birdcage"}

Compactificando a superfície mínima da figura 3.2 obtemos uma superfície de Riemann compacta $\bar{S}$ perfurada nos pontos correspondentes aos fins, ou seja, a superfície mínima é a imersão mínima de $\bar{S} \backslash\left\{p_{1}, \ldots, p_{5}\right\}$ em $\mathbb{R}^{3}$, onde $\bar{S}$ é o "birdcage" de gênero $n-1=2$ (compare a figura 3.2 e a figura 3.1 da esquerda).

O "birdcage" é invariante sob uma rotação de $\frac{360}{n}$ graus em torno do eixo $x_{3}$. Se indicarmos essa rotação por $\rho$ temos que

$$
\chi(\bar{S} / \rho)=\frac{\chi(\bar{S})}{n}+4 \frac{n-1}{n}=\frac{2-2(n-1)}{n}+4 \frac{n-1}{n}=2,
$$

onde $\chi(M)$ é a característica de Euler de $M$.

Assim, o quociente de $\bar{S}$ por $\rho$ é homeomorfo à esfera $\hat{\mathbb{C}}$. Podemos induzir uma estrutura complexa em $\bar{S} / \rho$ através da estrutura de $\bar{S}$ e temos portanto um biholomorfismo entre $\bar{S} / \rho$ e $\hat{\mathbb{C}}$, que é uma aplicação de Möbius, digamos $Z$. Em seguida podemos definir $f: \bar{S} \rightarrow \hat{\mathbb{C}}$ dada por $f=Z \circ \rho$, que é então um recobrimento ramificado de $n$ folhas de $\hat{\mathbb{C}}$.

Vamos então explicitar a função $Z$. Na figura 3.1 da esquerda o semi-eixo $x_{3}, x_{3} \geq$ 0 , corta a superfície num ponto $A$ e em seguida num ponto $B$. Podemos supor, identificando os pontos de $\bar{S}$ com suas classes de equivalência em $\bar{S} / \rho$, que $Z(A)=\xi$ e $Z(B)=1 / \xi$, para $\xi>1$ fixado. Já o semi-eixo $x_{1}, x_{1} \geq 0$ corta a superfície em um ponto $C$ e em seguida num ponto $D$, podemos supor $Z(C)=\infty$. Com isso determinamos a função $Z$ e conseqüentemente a função $f$.

Como $f$ é um recobrimento isométrico de $\widehat{\mathbb{C}}$, ela leva geodésicas de $\bar{S}$ em geodésicas de $\widehat{\mathbb{C}}$, ou seja, nos seus grandes círculos.

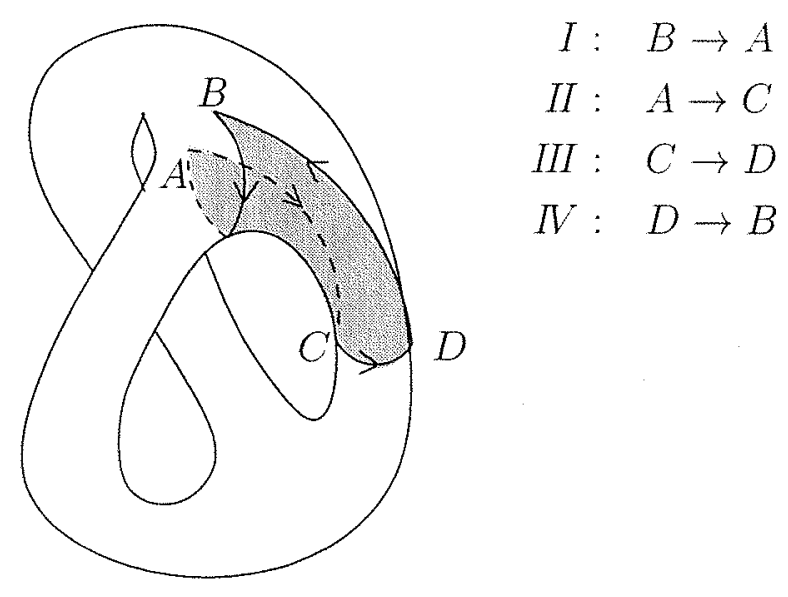

Figura 3.3: O "birdcage" e algumas curvas importantes.

A função $f$ tem ordem de ramificação $n-1$ em $B$ e $A$, ou seja, ela multiplica 
ângulos por $n$ em vizinhanças de $B$ e $A$. Assim, como $f(B)=1 / \xi$ e $f(A)=\xi$ temos que $f(I)=[1 / \xi, \xi]$ e como o ângulo entre $I$ e $I I$ é de $\frac{180}{n}$ graus temos que $f(I I) \subset \mathbb{R} \cup \infty$, mais precisamente $f(I I)=[\xi, \infty]$, pois $f(A)=\xi$ e $f(C)=\infty$. As curvas $I I$ e $I I I$ são perpendiculares em $C$ e portanto também o serão suas imagens sob $f$ pois $C$ não é ponto de ramificação para $f$. Logo $f(I I I) \subset i \mathbb{R}$. As curvas $I$ e $I V$ se interceptam em $B$ com ângulo de $\frac{180}{n}$ graus (como as curvas $I$ e $I I$ ). Portanto, $f(I V)$ é real e parte do valor $f(D)<1 / \xi$ até $f(B)=1 / \xi$, donde $f(D) \in \mathbb{R} \cap i \mathbb{R} \Rightarrow f(D)=0$. Portanto a região hachurada na figura 3.3 é levada na região de $\hat{\mathbb{C}}$ indicada na figura 3.4 .

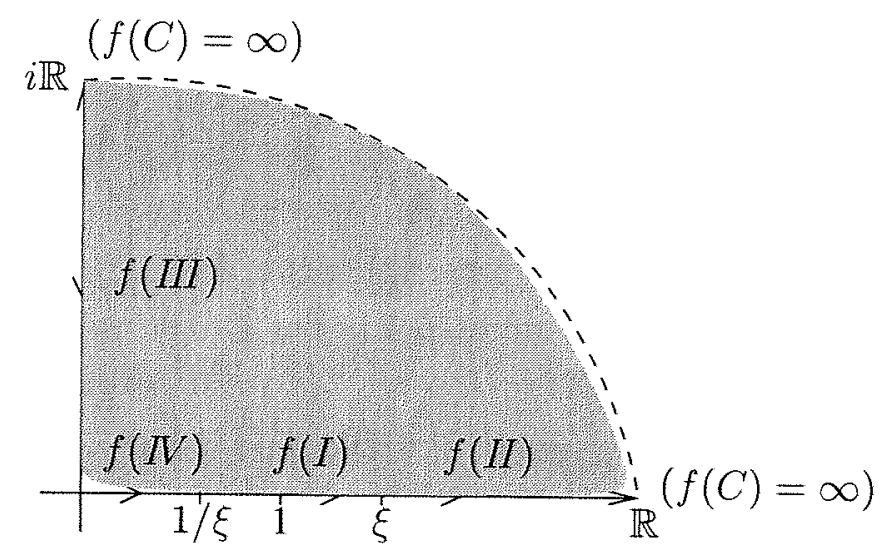

Figura 3.4: Imagem da função $f$.

Vamos agora explorar a simetria intrínseca do "birdcage" que foi citada no começo desta seção. Considere o quadrilátero hiperbólico da figura 3.5 .

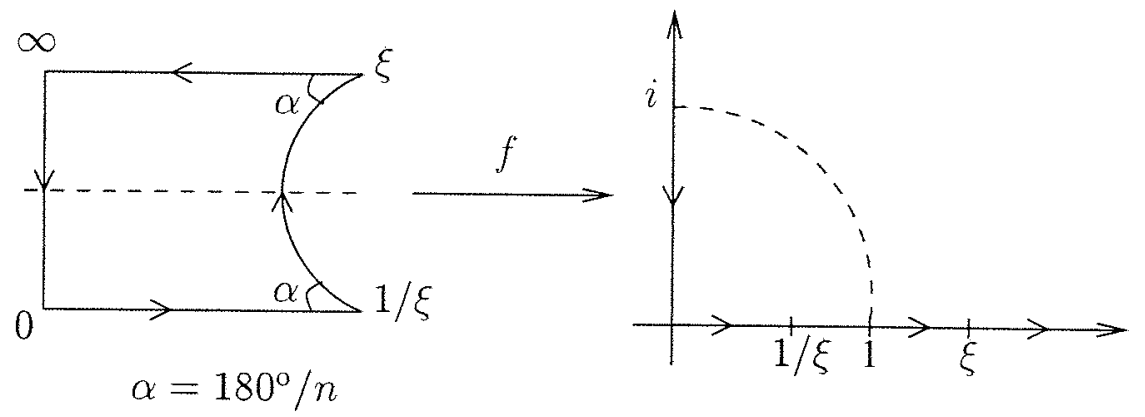

Figura 3.5: Simetria intrínseca do "birdcage".

Considere a pré-imagem da linha pontilhada na figura 3.5 da direita $(|f| \equiv 1)$, a qual é indicada pela linha pontilhada no quadrilátero da esquerda. A reflexão nessa linha pontilhada $(z \rightarrow 1 / \bar{z})$ seguida por uma reflexão no eixo imaginário $(z \rightarrow-\bar{z})$, que leva $f$ em $-1 / f$ dá uma involução em $\bar{S}$ cujos pontos fixos são os $p \in \bar{S}$ tais que $f(p)=-1 / f(p) \Rightarrow f^{2}(p)=-1 \Rightarrow f(p)= \pm i$. Agora, como $f$ é uma aplicação 
de recobrimento de $n$ folhas temos que tanto $i$ como $-i$ são assumidos $n$ vezes cada e portanto essa involução tem $2 n$ pontos fixos, todos com ordem 1 . Esta involução mostra que o "birdcage" é uma superfície de Riemann hiperelíptica (ou seja, ela possui uma involução cujos pontos fixos, que são os de ramificação, são simples e o quociente de $\bar{S}$ por esta involução é biholomorfo a $\hat{\mathbb{C}}$ ). De fato, se $*$ representa esta involução para o "birdcage" de gênero $n-1$ temos

$$
\chi(\bar{S} / *)=\frac{2-2(n-1)}{2}+2 n \frac{(2-1)}{2}=2 .
$$

Com argumento idêntico ao dado para a construção da função $f$, podemos definir $w: \bar{S} \rightarrow \hat{\mathbb{C}}$ satisfazendo $w(A)=\infty, w(B)=0$ e $w(C)=1$. Como $w$ também é um recobrimento isométrico de $\hat{\mathbb{C}}$ temos que sua imagem é dada pela figura 3.6.
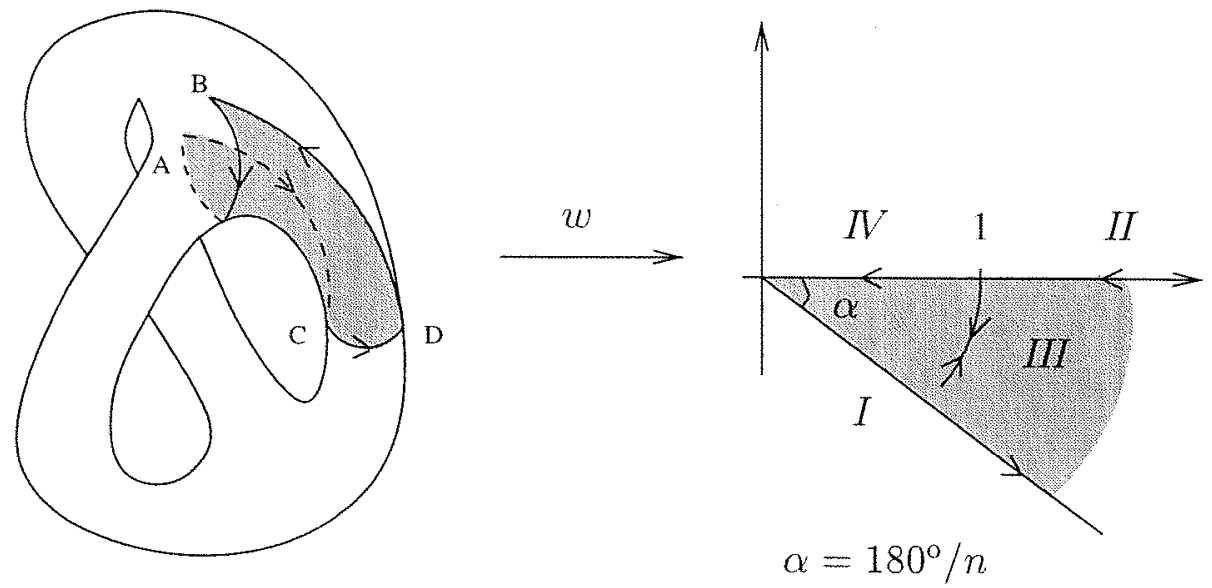

Figura 3.6: Imagem da função $w$.

A figura 3.6 é consistente, pois os pontos $A$ e $B$ não são pontos de ramificação para a função $w$. Existe um ponto de ramificação de ordem 1 no caminho III (um dos pontos fixos da involução discutida acima) entre os pontos $C$ e $D$ fazendo então com que $w(I I I)$ parta de $w(C)=1$, atinja algum valor no círculo unitário e volte para $w(D)=w(C)=1$, pois a involução hiperelíptica identifica os pontos $C$ e $D$.

Analisando os valores obtidos para as funções $f$ e $w$ podemos escrever uma tabela relacionando-os:

\begin{tabular}{c|c|c|c|c}
$\bar{S}$ & $A$ & $B$ & $C$ & $D$ \\
\hline$f$ & $\xi^{n}$ & $1 / \xi^{n}$ & $\infty^{n}$ & $0^{n}$ \\
\hline$w$ & $\infty$ & 0 & 1 & 1
\end{tabular}

Com isso podemos encontrar uma relação algébrica entre as funções $f$ e $w$ :

$$
w^{n}=\frac{(f-1 / \xi)(f+\xi)}{(f-\xi)(f+1 / \xi)}=\frac{f-1 / f+\xi-1 / \xi}{f-1 / f-\xi+1 / \xi} .
$$


A equação (3.1) define uma família de superfícies de Riemann compactas, cujo gênero é dado através da fórmula de Riemann-Hurwitz:

$$
\gamma=\frac{b}{2}-n+1=\frac{4(n-1)}{2}-n+1=n-1
$$

Precisamos ainda verificar que a equação acima representa de fato o "birdcage". Para isso basta verificar que às simetrias observadas no "birdcage" estão associadas involuções compatíveis com a equação algébrica:

- A involução hiperelíptica corresponde à transformação $f \rightarrow-1 / f$ que não altera a equação (3.1). Além disso temos que os pontos de ramificação de $w$ (ie, os pontos onde $d w=0$ ) são exatamente os pontos onde $f= \pm i$. De fato, se $k:=\xi-1 / \xi$ então

e portanto

$$
w=\left(\frac{f-1 / f+\xi-1 / \xi}{f-1 / f-\xi+1 / \xi}\right)^{\frac{1}{n}}
$$

$$
\begin{aligned}
d w & =0 \\
\Longleftrightarrow\left(f^{\prime}+f^{\prime} / f^{2}\right)(f-1 / f-k)-(f-1 / f+k)\left(f^{\prime}+f^{\prime} / f^{2}\right) & =0 \\
\Longleftrightarrow-2 k f^{\prime}-2 k f^{\prime} / f^{2} & =0 \\
\Longleftrightarrow f^{\prime}+f^{\prime} / f^{2} & =0 \\
\Longleftrightarrow f^{2} & =-1 \\
\Longleftrightarrow f & = \pm i
\end{aligned}
$$

Esses pontos de ramificação têm ordem 1 pois $w=0 \Longleftrightarrow f=-\xi$ ou $f=1$ / $\xi$. Mas como $f$ tem ordem de ramificação $n-1$ nesses pontos (e portanto multiplicidade $n$ ) temos que eles são zeros simples de $w$ e então todos os valores em $\hat{\mathbb{C}}$ são assumidos duas vezes, o que dá ordem de ramificação 1 para os pontos $f^{-1}(\{i,-i\})$.

- A reflexão dada pela curva $I$ corresponde a $f \rightarrow \bar{f}$ ou $w \rightarrow e^{-\frac{2 \pi i}{n}} \bar{w}$ que é consistente com a equação (3.1).

- As curvas $I I$ e $I V$ têm imagens reais tanto por $f$ quanto por $w$ e portanto as reflexões com relação a elas são dadas por $f \rightarrow \bar{f}$ e $w \rightarrow \bar{w}$ que também são consistentes com a equação (3.1).

- À curva $I I I$ corresponde a reflexão dada por $f \rightarrow-\bar{f}$ ou $w \rightarrow 1 / \bar{w}$ que são consistentes com a equação (3.1). 


\subsection{Os dados de Weierstrass para o "birdcage-ca- tenóide"}

Podemos agora usar as funções $f$ e $w$ para encontrar os dados de Weierstrass para o "birdcage-catenóide". As figuras 3.2 e 3.3 sugerem que o "birdcage-catenóide" é uma imersão mínima de $\bar{S}$ ("birdcage") perfurada nos pontos que dão origem aos fins catenoidais. Estes pontos são precisamente $f^{-1}(\{0, \pm \xi\})$.

Vamos então considerar $S:=\bar{S} \backslash f^{-1}(\{0, \pm \xi\}), \bar{S}$ definida pela equação (3.1) e $X: S \rightarrow \mathbb{R}^{3}$ a imersão mínima de $S$ cujo par de Weierstrass será indicado por $(g, d H)$. Lembramos que $g$ é dada pela composição da projeção estereográfica com a aplicação normal de Gauss e a imersão $X$. A diferencial $d H$ pode ser descrita em termos dos tipos de fins que queremos para a superfície e do comportamento de objetos intrínsecos à superfície, como por exemplo a métrica.

Inspirados pela técnica de adição de asas vista anteriormente vamos normalizar a funcão $f$ definindo $h:=\xi f$ e $x:=\xi^{2}$. Desse modo temos $h=1$ no fim catenoidal superior de $X(S), h=-1$ no fim inferior, $h=x$ na sela superior e $h=-x$ na sela inferior. Na curva $I I I$ indicada na figura 3.3 onde $f$ é imaginária pura, $h$ também o é. Deste modo, a função $h$ tem o mesmo comportamento das funções $h$ do capítulo anterior. Podemos então enxergar os "birdcage-catenóide"s de gênero $n-1$ como $n+2$-nóides (imersões mínimas da esfera perfurada com $n+2$ fins catenoidais, sendo 2 verticais e $n$ horizontais), onde empurramos $n$ asas para o interior formando as selas (onde $h=x$ e $h=-x$ ). Reescrevendo a equação algébrica do "birdcage" em termos da função $h$ temos

$$
w^{n}=\left(\frac{h+x}{h-x}\right)\left(\frac{h-1}{h+1}\right)
$$

Considerando a superfície $X(S)$ representada na figura 3.2 e seu vetor normal unitário temos que no seu fim catenoidal superior o vetor normal é vertical e podemos escolhê-lo apontando para baixo, donde $g(B)=0$. Temos ainda um outro ponto onde o vetor normal é vertical. Este ponto está sobre a imagem do caminho $N$ e o vetor aponta para cima, ou seja existe $y \in(0,1)$ tal que $h=y \Rightarrow g=\infty$.

Na sela superior, correspondente ao ponto $A$ em $\bar{S}$, temos que o vetor normal é vertical e aponta para baixo, donde $g(A)=0$. Mas esse é um ponto de ramificação para $g$, e devido à simetria rotacional de $360 / n$ graus vista anteriomente, temos que $g$ tem multiplicidade $n-1$ numa vizinhança de $A$ e portanto ordem de ramificação $n-2$. Os outros zeros e pólos de $g$ podem ser localizados devido à essa simetria rotacional e à simetria reflexiva no plano $x_{3}=0$. A figura 3.7 ilustra a situação. 


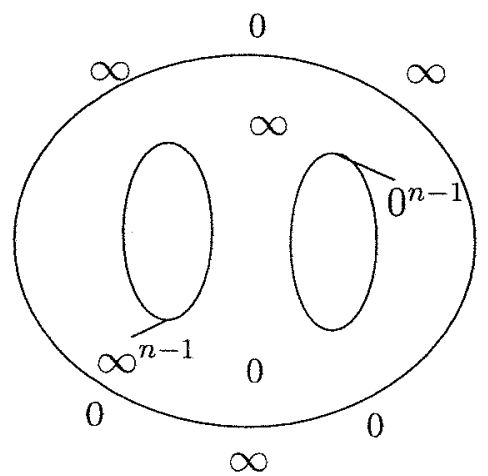

Função $g$

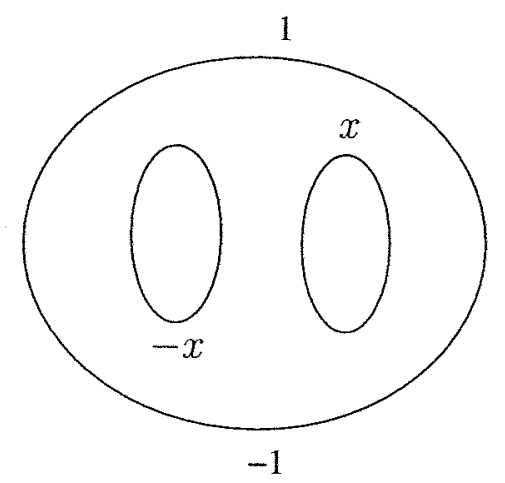

Função $h$

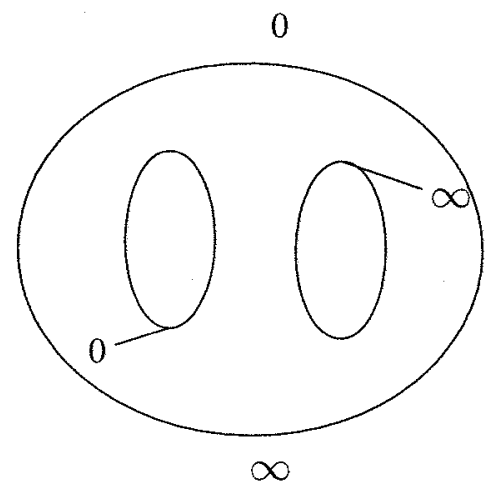

Função $w$

Figura 3.7: Zeros e pólos de $g, h$ e $w$ com suas multiplicidades.

Com base na figura 3.7 podemos escrever uma relação algébrica de $g \operatorname{com} h$ e $w$ :

$$
g=k\left(\frac{h+y}{h-y}\right)\left(\frac{x-h}{x+h}\right) w .
$$

O fator $k \in \mathbb{C}$ aparece pois a equação foi escrita considerando apenas os zeros e pólos das funções $g, h$ e $w$. Mas levando em conta que o vetor normal de $X(S)$ no ponto correpondente a $C$ em $\bar{S}$ satisfaz $g(C)=-1, h(C)=\infty$ e $w^{n}(C)=1 \Rightarrow$ $w(C)=\sqrt[n]{1}=1$ temos que $k=1$. (os valores $g(C)=-1 \sqrt[n]{1}$ dão os $n$ valores de $g$ em cada um dos fins horizontais). Assim temos a equação (3.3):

$$
g=\left(\frac{h+y}{h-y}\right)\left(\frac{x-h}{x+h}\right) w .
$$

A função $g$ também é uma aplicação de recobrimento de $\hat{\mathbb{C}}$ e seu número de folhas, $\operatorname{deg}(g)$, é dado pela fórmula de Jorge Meeks (ver [7]):

$$
\operatorname{deg}(g)=k+r-1=n-1+n+2-1=2 n,
$$

que é o esperado se observarmos a figura 3.2 (no caso da figura temos $n=2$ e portanto $\operatorname{deg}(g)=6$, e podemos verificar que de fato os zeros e pólos de $g$ são assumidos 6 vezes, contando as multiplicidades).

A equação (3.3) representa com consistência os valores do vetor normal ao longo dos caminhos $I, I I, I I I$ e $I V$ como vemos a seguir:

(I) $g$ deve estar sobre o meridiano que forma ângulo de $180 / n$ graus com o eixo $x_{1}$ tal que $x_{2} \leq 0$ e de fato, $\left.h\right|_{I} \in[1, x]$ e $w(I)=e^{-\frac{i \pi}{n}} t, t \in[0, \infty]$.

(II) $g$ deve ser real e de fato $\left.h\right|_{I I} \in[x, \infty]$ e $\left.w\right|_{I I} \in[1, \infty]$.

(III) $g$ deve ser unitária e de fato $\left.h\right|_{I I I} \in i \mathbb{R}_{+}$e $\left.w\right|_{I I I} \in S^{1}$. 
$(N) g$ deve ser real e de fato temos $\left.h\right|_{N} \in[0,1]$ e $\left.w\right|_{N} \in[0,1]$.

A equação obtida para $g$ também reflete o comportamento esperado de acordo com as simetrias de $X(S)$ :

- A reflexão na curva $I$ é dada por $w \rightarrow e^{-\frac{2 \pi i}{n}} \bar{w}$ e $h \rightarrow \bar{h}$ o que leva $g$ em $e^{-\frac{2 \pi i}{n}} \bar{g}$ que é o comportamento esperado para $g$ por uma reflexão nessa curva.

- As reflexões relativamente às cuvas $I I$ e $I V$ são dadas por $w \rightarrow \bar{w}$ e $h \rightarrow \bar{h}$ donde $g \rightarrow \bar{g}$.

- A reflexão com relação à curva $I I I$ é dada por $w \rightarrow 1 / \bar{w}$ e $h \rightarrow-\bar{h}$, o que dá

$$
\left(\frac{-\bar{h}+y}{-\bar{h}-y}\right)\left(\frac{x+\bar{h}}{x-\bar{h}}\right)\left(-\frac{1}{\bar{w}}\right)=\left(-\frac{\overline{h-y}}{\overline{h+y}}\right)\left(\frac{\overline{x+h}}{\overline{x-h}}\right)\left(\frac{1}{\bar{w}}\right)=-\frac{1}{\bar{g}},
$$

consistente com o comportamento de $g$.

Para encontrar a expressão da diferencial $d H$, precisamos determinar seus zeros e pólos, levando em conta as características desejadas para a superfície. Faremos isto considerando os zeros e pólos da função $g$ obtida na equação 3.3.

Nos fins catenoidais verticais temos que $g=0$ e $g=\infty$ (simples) e portanto $d H$ deve ter pólos simples nesses pontos (pois a métrica nesses pontos não é regular e queremos crescimento logarítmico na terceira coordenada da representação de Weierstrass para obtermos fins catenoidais). Nos fins catenoidais horizontais $g$ é finita e não nula e portanto devemos ter pólos de multiplicidade 2 para $d H$. Por exemplo o fim na direção do eixo $x_{1}$ na figura 3.2 tem $g=-1$ e queremos crescimento logarítimico na primeira coordenada, $(g-1 / g) d H$. Mas ali $g-1 / g=0$ e portanto $d H=\infty^{2}$, que é compativel com as outras coordenadas.

Nos pontos de sela, temos $g=0$ e $g=\infty$, com multiplicidade $n-1$ cada, e como esses são pontos regulares para a métrica da superfície, $d H$ deve ter zeros de ordem $n-1$ em cada uma das selas. Nos outros pontos onde $g=0$ ou $g=\infty$ a diferencial $d H$ também deve ter zeros de modo a cancelar os pólos criados por $g \pm 1 / g$ na representação de Weierstrass. Esses pontos são exatamente os pontos $h= \pm y$, que foram identificados na obtenção da função $g$. A figura 3.8 resume a situação dos zeros e pólos de $d H$.

Com base nas figuras 3.8 e 3.7 podemos escrever

$$
d H=c \frac{(h-y)(h+y)}{h^{2}} \frac{d h}{1-h^{2}}=c \frac{h^{2}-y^{2}}{h^{2}} \frac{d h}{1-h^{2}}, c \in \mathbb{C} .
$$

Para determinar a constante $c$ basta observarmos que ao longo da curva III a terceira coordenada da imersão $X(\bar{S})$ é constante e portanto $\left.d H\right|_{I I I} \in i \mathbb{R}$ e portanto 


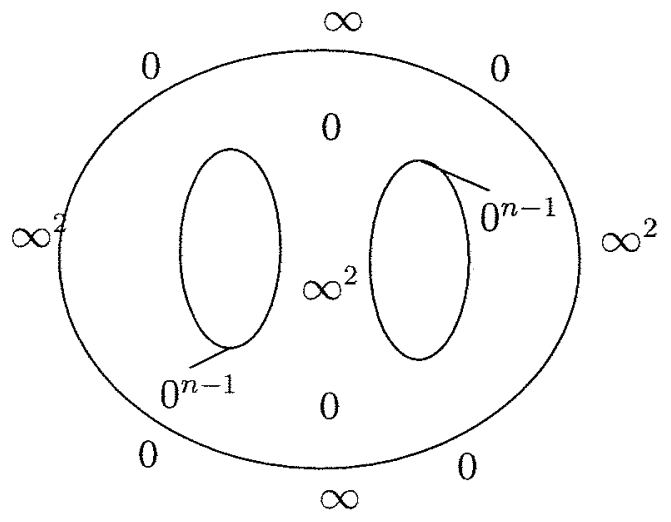

Figura 3.8: Zeros e pólos de $d H$ com suas multiplicidades.

$c \in \mathbb{R}$. Podemos escolher $c=1$, pois ela representa apenas uma dilatação nos três eixos e a equação de superfícies mínimas é invariante por dilatações.

Com isso obtemos os dados de Weierstrass para o "birdcage-catenóide":

$$
\begin{aligned}
g & =\frac{h+y}{h-y} \frac{x-h}{x+h} w \\
d H & =\frac{h^{2}-y^{2}}{h^{2}} \frac{d h}{h^{2}-1}
\end{aligned}
$$

Precisamos mostrar agora que os dados de Weierstrass de 3.4 e 3.5 nos dão as simetrias que o "birdcage-catenóide" tem. Da figura 3.2 vemos que a superfície mínima tem apenas geodésicas planas, que dão as simetrias reflexivas nos planos que as contém. Essas geodésicas são exatamente as imagens das curvas $I, I I, I I I$ e $N$ :

(I) $\left.h\right|_{I} \in[1, x], g^{n}<0$ e $\left.d H(\dot{h}) \in \mathbb{R} \Rightarrow \frac{d g}{g} d H\right|_{\dot{h}} \in \mathbb{R}$

(II) $\left.h\right|_{I I} \in[x, \infty],-1 \leq g \leq 0$ e $\left.d H(h) \in \mathbb{R} \Rightarrow \frac{d g}{g} d H\right|_{h} \in \mathbb{R}$

$\left.(I I I) h\right|_{I I I} \in i \mathbb{R}, g \in S^{1}$ e $\left.d H(\dot{h}) \in i \mathbb{R} \Rightarrow \frac{d g}{g} d H\right|_{\dot{h}} \in \mathbb{R}$

$\left.(N) h\right|_{N} \in[y, 1], 0 \leq g \leq \infty$ e $\left.d H(\dot{h}) \in \mathbb{R} \Rightarrow \frac{d g}{g} d H\right|_{\dot{h}} \in \mathbb{R}$

Portanto, as imagens das curvas $I, I I, I I I$ e $I V$ são de fato geodésicas planas e os planos que as contém são planos de simetria para o "birdcage-catenóide". 


\subsection{O problema dos períodos}

Para concluir, precisamos mostrar que a superfície mínima $X(S)$ não tem períodos reais, como esperamos a partir do esboço na figura 3.2. É suficiente, para isto, mostrar que $\int_{\gamma}\left(\phi_{1}, \phi_{2}, \phi_{3}\right)$ é imaginária pura para toda curva fechada $\gamma$ em $\bar{S}$, onde $\phi_{i}$ são as coordenadas da representação de Weierstrass.

No "birdcage" vamos analisar curvas fechadas em torno dos pontos removidos. Devido às simetrias mostradas anteriormente, a análise é suficiente nos pontos $B$ e $D$, pois o comportamento é idêntico nos outros pontos. $O$ ponto $B$ é a intersecção das curvas $I$ e $W$ que dão origem a planos de simetria para a superfície $X(S)$. Portanto, qualquer curva fechada em torno de $B$ é homotópica a uma outra curva fechada em torno de $B$ que é invariante pelas reflexões nas curvas $I$ e $I V$ e o valor de $\Re \int\left(\phi_{1}, \phi_{2}, \phi_{3}\right)$ é idêntico sobre qualquer uma das duas curvas. Com isso o vetor período em $\mathbb{R}^{3}$ é simultaneamente perpendicular aos planos de simetria gerados pelas curvas $I$ e $I V$. Esses planos não são paralelos pois o vetor normal ao longo dessas curvas não o é. Assim, o vetor período para o fim catenoidal superior se anula (conseqüentemente o mesmo ocorre para o fim catenoidal inferior). O mesmo argumento pode ser aplicado ao ponto $D$ lembrando que $D=I I I \cap I V$. Com isso mostramos que o período real se anula em todos os fins da superfície.

Falta agora uma análise dos períodos nas curvas geradoras da homologia de $\bar{S}$. Mas, novamente devido às simetrias de $\bar{S}$, basta considerarmos o periodo em apenas duas curvas.

A primeira é uma componente conexa do levantamento de $h \circ I I$ por $h$. Vemos que esse levantamento intercepta as curvas $I$ e $I I I$ e portanto o vetor período deve ser perpendicular à imagem dessas duas curvas o que faz com que ele se anule.

A outra é uma curva qualquer que seja homotópica à curva $\gamma$ indicada na figura 3.9 junto com sua reflexão com relação à curva $I I$ ou $I V$.

Devido à simetria com relação à curva $N$ temos que o vetor período é perpendicular ao plano $x_{2}=0$ e portanto é suficiente mostrar que $\Re \int_{\gamma} \phi_{2}=0$.

Vamos então mostrar que para certos valores de $x$ e $y$ nas equações 3.4 e 3.5 o período em questão se anula. Mais precisamente vamos mostrar que para cada $n \geq 2$ valem as seguintes afirmações:

- Existe um único $x_{n}>1$ tal que para todo $x \in\left[1, x_{n}\right]$ existem dois valores reais $y_{1}(x)$ e $y_{2}(x)$ para os quais o período se anula. Se $x>x_{n}$ o problema de períodos não tem solução.

- As funções $y_{1}(x)$ e $y_{2}(x)$ são respectivamente crescente e decrescente com $x$. 


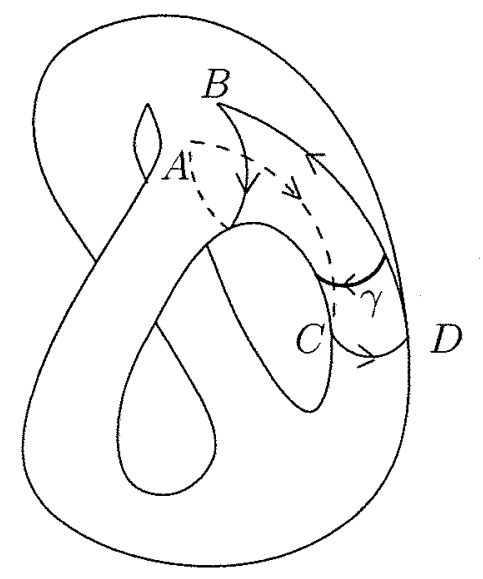

Figura 3.9: O "birdcage" e a curva $\gamma$.

Ambas são contínuas e $y_{1}(x) \leq y_{2}(x), x \in\left[1, x_{n}\right]$. A igualdade só ocorre quando $x=x_{n}$.

- $\lim _{x \rightarrow 1} y_{1}(x)=0$ e $\lim _{x \rightarrow 1} y_{2}(x)=1$

Para isso definimos

$$
P(n, x, y)=-2 \Re \int_{\gamma} \phi_{2}
$$

lembrando que $\phi_{2}=i / 2(g+1 / g) d H$. O sinal de menos na expressão de $P$ simplificará muitas das contas no que se segue. Das figuras 3.4 e 3.5 vemos que as curvas $I, I I, I I I$ e $\Pi$ formam um quadrilátero cujo interior é levado na região $\{z \in \hat{\mathbb{C}}: 0<\arg (z)<\pi /$ $2\}$ por $f$ ou por $h$. As transformações de Möbius $\frac{x-h}{x+h}, \frac{1-h}{1+h}$ e suas inversas levam esse quadrante em abertos de $\hat{\mathbb{C}}$ que excluem o semi-eixo real negativo. Portanto o ramo da raiz $n$-ésima dado por $\sqrt[n]{e^{i t}}:=e^{i t / n}$ está bem definido e é contínuo nessa região.

Fixado esse ramo definimos

$$
F(x, h)=\left(\frac{x-h}{x+h}\right)^{1-\frac{1}{n}}\left(\frac{1-h}{1+h}\right)^{\frac{1}{n}}
$$

Podemos então escrever os dados de Weierstrass do "birdcage-catenóide" em termos de $F(x, h)$ e substituí-los na expressão de $P(n, x, y)$ : 


$$
\begin{aligned}
P(n, x, y) & =-2 \Re \int_{\gamma} \phi_{2} \\
& =-2 \Re \int_{\gamma} i\left(g+\frac{1}{g}\right) d H \\
& =-2 \Re \int_{\gamma} i\left(\frac{h+y}{h-y} F(x, h)+\frac{h-y}{h+y} \frac{1}{F(x, h)}\right) \frac{h^{2}-y^{2}}{h^{2}} \frac{d h}{1-h^{2}} \\
& =-2 \Re \int_{\gamma} i\left((h+y)^{2} F(x, h)+(h-y)^{2} \frac{1}{F(x, h)}\right) \frac{d h}{h^{2}\left(1-h^{2}\right)} \\
& =2\left(y^{2} \Re \int_{\gamma} \frac{F+1 / F}{i h^{2}\left(1-h^{2}\right)} d h+2 y \Re \int_{\gamma} \frac{F-1 / F}{i h\left(1-h^{2}\right)} d h+\Re \int \frac{F+1 / F}{i\left(1-h^{2}\right)} d h\right)
\end{aligned}
$$

Como queremos analizar $P=0$ podemos considerar

$$
P=y^{2} \Re \int_{\gamma} \frac{F+1 / F}{i h^{2}\left(1-h^{2}\right)} d h+2 y \Re \int_{\gamma} \frac{F-1 / F}{i h\left(1-h^{2}\right)} d h+\Re \int_{\gamma} \frac{F+1 / F}{i\left(1-h^{2}\right)} d h .
$$

Fixando os valores de $n$ e $x$ a equação acima é a de uma parábola em $y$. Definindo

$$
\begin{aligned}
a(n, x) & :=\Re \int_{\gamma} \frac{F+1 / F}{i h^{2}\left(1-h^{2}\right)} d h, \\
b(n, x) & :=2 \Re \int_{\gamma} \frac{F-1 / F}{i h\left(1-h^{2}\right)} d h, \\
c(n, x) & :=\Re \int_{\gamma} \frac{F+1 / F}{i\left(1-h^{2}\right)} d h .
\end{aligned}
$$

temos $P=a y^{2}+b y+c$.

Vamos agora obter alguns resultados importantes sobre as funções $a, b$ e $c$ de (3.9), (3.10) e (3.11).

Proposição 3.3.1. As funções a,b e c definidas em (3.9), (3.10) e (3.11) satisfazem as seguintes propriedades:

(a) b(n,x) é negativa e crescente em $x$;

(b) $0<-b(n, x)<2 a(n, x), \forall x>1$ (o vértice da parábola está entre 0 e 1);

(c) $a(n, x)>0$ e decrescente em $x$

(d) $c(n, x)>0$ e crescente em $x$; 
(e) $a(n, x), b(n, x)$ e c(n,x) são contínuas em $x=1$. Além disso, $\left.c\right|_{x=1}=0 e$ $1+\left.\frac{b+c}{a}\right|_{x=1}=0$.

(f) $(a+b+c)(n, x)$ é positiva e crescente em $x$.

\section{Demonstração:}

(a) Cálculos diretos mostram que

$$
\lim _{h \rightarrow 0} \frac{F-1 / F}{h}=-4\left(\frac{x+n-1}{n x}\right)<\infty, x>1
$$

portanto o integrando de $b(n, x)$ é holomorfo quando $h=0$, ie, no ponto $D$ na figura 3.3. Assim o valor da integral não se altera se trocarmos $\gamma$ pela curva $\tilde{\gamma}$ cuja imagem por $h$ é $i \mathbb{R}_{+}, h(t)=i t, 0<t<\infty$ e então

$$
b(n, x)=2 \Re \int_{0}^{\infty} \frac{(F-1 / F) \circ h(t)}{i i t\left(1-(i t)^{2}\right)} i d t=2 \Re \int_{0}^{\infty} \frac{(F-1 / F) \circ h(t)}{i t\left(1+t^{2}\right)} d t .
$$

Mas $|F \circ h(t)|=1$ e portanto $F-1 / F=2 i \Im F$. A parte imaginária de $(F \circ h)$ é negativa pois $\left.F\right|_{h=i t} \in S^{1} \operatorname{com} F(x, 0)=1$ e $\Im F^{1-1 / n}(x, i)=\frac{1-x^{2}}{1+x^{2}}<0 \Rightarrow$ $\Im F(x, i)<0$ donde $\left.\Im F\right|_{h=i t}<0$. Portanto a expressão de $F$ em (3.7) sobre $h(t)=i t$ tem seu argumento variando entre $-\pi / 2$ e $\pi / 2$. Então segue-se que $b(n, x)<0$. Para mostrar que $b$ é crescente vamos mostrar que $\frac{\partial b}{\partial x}>0$, e para tanto são necessárias as seguites relações:

$$
\begin{aligned}
\frac{\partial F}{\partial x} & =\left(\frac{1-h}{1+h}\right)^{1 / n}(1-1 / n)\left(\frac{x-h}{x+h}\right)^{-1 / n}\left(\frac{x+h-(x-h)}{(x+h)^{2}}\right) \\
& =2(1-1 / n)\left(\frac{1-h}{1+h}\right)^{n}\left(\frac{x-h}{x+h}\right)^{-1 / n}\left(\frac{h}{(x+h)^{2}}\right) \\
& =2(1-1 / n) \frac{h}{x^{2}-h^{2}}\left(\frac{1-h}{1+h}\right)^{n}\left(\frac{x-h}{x+h}\right)^{1-1 / n} \\
& =2(1-1 / n) \frac{h}{x^{2}-h^{2}} F .
\end{aligned}
$$

Ou seja,

$$
\frac{\partial F}{\partial x}=2(1-1 / n) \frac{h}{x^{2}-h^{2}} F
$$


Analogamente temos

$$
\frac{\partial(1 / F)}{\partial x}=-2(1-1 / n) \frac{h}{x^{2}-h^{2}} 1 / F
$$

Derivando sob o sinal de integração e usando as expressões acima temos

$$
\begin{aligned}
\frac{\partial b}{\partial x} & =2 \Re \int_{0}^{\infty} \frac{\partial}{\partial x}[(F-1 / F) \circ h(t)] \frac{d t}{i t\left(1+t^{2}\right)} \\
& =2 \Re \int_{0}^{\infty} 2(1-1 / n) \frac{h(t)}{x^{2}-h^{2}(t)}(F+1 / F) \circ h(t) \frac{d t}{i t\left(1+t^{2}\right)} \\
& =4(1-1 / n) \Re \int_{0}^{\infty} \frac{i t}{x^{2}+t^{2}}(F+1 / F) \circ h(t) \frac{d t}{i t\left(1+t^{2}\right)} \\
& =4(1-1 / n) \Re \int_{0}^{\infty} \frac{(F+1 / F) \circ h(t)}{\left(1+t^{2}\right)\left(x^{2}+t^{2}\right)} d t .
\end{aligned}
$$

Mas como $|(F+1 / F) \circ h(t)| \equiv 1$ temos que $F+1 / F=2 \Re F$, quando $h(t)=$ it, $0<t<\infty$. Logo,

$$
\begin{aligned}
\frac{\partial(\Re F)}{\partial x} & =\frac{1}{2}\left(\frac{\partial F}{\partial x}+\frac{\partial(1 / F)}{\partial x}\right) \\
& =(1-1 / n) \frac{h}{x^{2}-h^{2}}(F-1 / F) \\
& =(1-1 / n) \frac{i t}{x^{2}+t^{2}} 2 i \Im F \\
& =-2(1-1 / n) \frac{t}{x^{2}+t^{2}} \Im F>0,
\end{aligned}
$$

pois $0<t<\infty$ e $\Im F<0$. Com isso $\Re F$ é uma função crescente de $x$. Se mostrarmos então que $\left.\frac{\partial b}{\partial x}\right|_{x=1}>0$, então teremos $\frac{\partial b}{\partial x}>0, \forall x \geq 1$.

Cálculos diretos mostram que

$$
\begin{aligned}
\left.\frac{\partial b}{\partial x}\right|_{x=1} & =4(1-/ n) \Re \int_{0}^{\infty}\left[\frac{1-i t}{1+i t}+\frac{1+i t}{1-i t}\right] \frac{d t}{\left(1+t^{2}\right)\left(1+t^{2}\right)} \\
& =8(1-1 / n) \Re \int_{0}^{\infty} \frac{1-t^{2}}{\left(1+t^{2}\right)\left(1+t^{2}\right)^{2}} d t
\end{aligned}
$$

Dividindo o intervalo de integração em $(0,1]$ e $[1, \infty)$ e fazendo a mudança $t \rightarrow 1 / t$ na integral sobre $[1, \infty)$ obtemos

$$
\left.\frac{\partial b}{\partial x}\right|_{x=1}=8(1-1 / n)\left[\int_{0}^{1} \frac{1-t^{2}}{\left(1+t^{2}\right)^{3}} d t-\int_{0}^{1} \frac{1-t^{2}}{\left(1+t^{2}\right)^{2}\left(1+1 / t^{2}\right)} d t\right]
$$


Mas para $t \in(0,1)$ temos $t<1 / t$ donde a primeira integral é maior que a segunda e portanto $\left.\frac{\partial b}{\partial x}\right|_{x=1}>0$. Lembrando que $\Re F$ é crescente com $x$ temos $\frac{\partial b}{\partial x}>0, \forall x \geq 1$

(b)

$$
\begin{aligned}
a+\frac{b}{2} & =\Re \int_{\gamma} \frac{F+F^{-1}}{i h^{2}\left(1-h^{2}\right)} d h+\Re \int_{\gamma} \frac{F-F^{-1}}{i h\left(1-h^{2}\right)} \\
& =\Re \int_{\gamma}\left[\frac{F}{i h\left(1-h^{2}\right)}\left(\frac{1}{h}+1\right)+\frac{F^{-1}}{i h\left(1-h^{2}\right)}\left(\frac{1}{h}-1\right)\right] \\
& =\Re \int_{\gamma}\left[\frac{F}{i h^{2}(1-h)}+\frac{F^{-1}}{i h^{2}(1+h)}\right] d h \\
& =\Re \int_{\gamma} \frac{F}{i h^{2}(1-h)} d h+\Re \int_{\gamma} \frac{F^{-1}}{i h^{2}(1+h)} d h .
\end{aligned}
$$

Podemos aqui escolher $\gamma$ tal que $h \circ \gamma=t, 1<t<x$, pois esta curva é homotópica à curva original. Nesse caso temos

$$
\begin{aligned}
F(h(t)) & =\left(\frac{x-t}{x+t}\right)^{1-\frac{1}{n}}\left(\frac{1-t}{1+t}\right)^{\frac{1}{n}} \\
& =\left(\frac{x-t}{x+t}\right)^{1-\frac{1}{n}}\left(\frac{t-1}{t+1}\right)^{\frac{1}{n}} \sqrt[n]{-1}
\end{aligned}
$$

Vamos escolher aqui o valor $e^{-i \pi / n}$ para $\sqrt[n]{-1}$, pois assim a função $F$ fica contínua e então

$$
F(h(t))=\left(\frac{x-t}{x+t}\right)^{1-\frac{1}{n}}\left(\frac{t-1}{t+1}\right)^{\frac{1}{n}} e^{-i \frac{\pi}{n}}
$$

De (3.15) vem que

$$
\Re \frac{F \circ h}{i t^{2}(1-t)}=\Im \frac{F \circ h}{t^{2}(t-1)}=\frac{\sin (\pi / n)|F \circ h|}{t^{2}(t-1)}
$$

e

$$
\Re \frac{(F \circ h)^{-1}}{i t^{2}(t+1)}=\Im \frac{(F \circ h)^{-1}}{t^{2}(t+1)}=\frac{\sin (\pi / n)\left|(F \circ h)^{-1}\right|}{t^{2}(t+1)} .
$$

Portanto,

$$
a+\frac{b}{2}=\sin \left(\frac{\pi}{n}\right)\left\{\int_{1}^{x} \frac{|F \circ h|}{t^{2}(t-1)} d t+\int_{1}^{x} \frac{\left|(F \circ h)^{-1}\right|}{t^{2}(t+1)} d t\right\} .
$$


Como os integrandos são positivos em (3.16) temos que $a+b / 2>0$ para todo $x>1$, donde $0<-b<2 a$.

(c) Do item (b) acima temos que $a(n, x)>0$. Vamos mostrar agora que $\frac{\partial a}{\partial x}<0$. Usando as expressões de $\frac{\partial F}{\partial x}$ e $\frac{\partial F^{-1}}{\partial x}$ de (3.13) e (3.14) temos que

$$
\begin{aligned}
\frac{\partial a}{\partial x} & =\Re \int_{\gamma} \frac{\partial}{\partial x}\left(F+F^{-1}\right) \frac{d h}{i h^{2}\left(1-h^{2}\right)} \\
& =2(1-1 / n) \Re \int_{\gamma} \frac{F-F^{-1}}{i h\left(x^{2}-h^{2}\right)\left(1-h^{2}\right)} d h .
\end{aligned}
$$

No item (a) mostramos que $\lim _{h \rightarrow 0} \frac{F-F^{-1}}{h}$ é finito e portanto o integrando acima é holomorfo em $h=0$, permitindo a escolha de $\gamma$ tal que $h \circ \gamma=i t, 0<t<\infty$ sem alterar o valor da integral. Vimos também no item (a) que sobre essa curva temos $F-F^{-1}=2 i \Im F$, com $\Im F<0$ donde $\frac{\partial a}{\partial x}<0$.

(d) O integrando em (3.11) é holomorfo em $h=0$ e portanto podemos considerar $\gamma$ tal que $h \circ \gamma=i t, 0<t<\infty$ e então

$$
c(n, x)=\int_{0}^{\infty} \frac{\left(F+F^{-1}\right)(h(t))}{1+t^{2}} d t
$$

Como vimos no item (a) desta proposição $\left(F+F^{-1}\right)(h(t))=2 \Re F(h(t))$ que é crescente em $x$. No item (e) mostraremos que $c(n, x)$ é contínua na variável $x$. Portanto, se mostrarmos que $\left.c\right|_{x=1} \geq 0$ então $c(n, x)$ será positiva e crescente para todo $x>1$, pois o integrando em (3.17) é crescente em $x$. Para verificarmos isso, basta ver que fazendo $x=1$ em (3.7) temos

$$
F(1, t)=\frac{1-t}{1+t}
$$

Portanto,

$$
F+F^{-1}(1, t)=2 \frac{1+t^{2}}{1-t^{2}}
$$

que substituído em (3.17) resulta

$$
\left.c\right|_{x=1}=2 \int_{0}^{\infty} \frac{1+t^{2}}{1-t^{2}} \frac{d t}{1-t^{2}}=2 \int_{0}^{\infty} \frac{d t}{1-t^{2}} .
$$

Dividindo o intervalo de integração em $(0,1]$ e $[1, \infty)$ e fazendo a mudança $t \rightarrow 1 / t$ na segunda integral temos 


$$
\begin{aligned}
\left.c\right|_{x=1} & =2 \int_{0}^{\infty} \frac{d t}{1-t^{2}}=2\left\{\int_{0}^{1} \frac{d t}{1-t^{2}}+\int_{1}^{\infty} \frac{d t}{1-t^{2}}\right\} \\
& =2\left\{\int_{0}^{1} \frac{d t}{1-t^{2}}+\int_{1}^{0} \frac{-d t / t^{2}}{1-1 / t^{2}}\right\} \\
& =2\left\{\int_{0}^{1} \frac{d t}{1-t^{2}}-\int_{1}^{0} \frac{d t}{t^{2}-1}\right\}=0 .
\end{aligned}
$$

(e) Considere o quadrante $Q=\{z \in \hat{\mathbb{C}}: 0<\arg (z)<\pi / 2\}$ e $K \subset \bar{Q}$ compacto tal que $K \cap\{0,1, x\}=\emptyset(0,1$ e $x$ são os pólos dos integrandos de $a, b$ e $c$ em (3.9), (3.10) e (3.11)).

Da expressão de $F$ em (3.7) temos que $\lim _{x \rightarrow 1}=\frac{1-h}{1+h}$. Podemos ainda escolher $K$ tal que a imagem de $\gamma$ esteja contida em $K$. Assim as funções $a, b$ e $c$ em (3.9), (3.10) e (3.11) estão definidas e são contínuas em $x=1$. No item (d) mostramos que $\left.c\right|_{x=1}=0$. Vamos agora analizar $\frac{b+c}{a}$.

Multiplicando $b$ por $h / h$ e $c$ por $h^{2} / h^{2}$ e rearranjando os termos em (3.8) temos

$$
P=\Re \int_{\gamma} \frac{(h+y)^{2} F+(h-y)^{2} F^{-1}}{i h^{2}\left(1-h^{2}\right)} d h
$$

Se $x=1$ temos

$$
\begin{aligned}
\frac{(h+y)^{2} F}{h^{2}\left(1-h^{2}\right)} & =\frac{1}{h^{2}}\left(\frac{h+y}{1+h}\right)^{2}=\frac{A_{1}}{h}+\frac{B_{1}}{1+h}+\frac{C_{1}}{h^{2}}+\frac{D_{1}}{(1+h)^{2}}, \mathrm{e} \\
\frac{(h-y)^{2} F^{-1}}{h^{2}\left(1-h^{2}\right)} & =\frac{1}{h^{2}}\left(\frac{h-y}{h-1}\right)^{2}=\frac{A_{1}}{h}+\frac{B_{1}}{h-1}+\frac{C_{1}}{h^{2}}+\frac{D_{1}}{(h-1)^{2}} .
\end{aligned}
$$

Podemos encontrar os valores das constantes $A_{i}, B_{i}, C_{i} D_{i}, i=1,2$ observando que a primeira expressão acima é equivalente a

$$
h^{2}+2 h y+y^{2}=A_{1} h(1+h)^{2}+B_{1} h^{2}(1+h)+C_{1}(1+h)^{2}+D_{1} h^{2} .
$$

Na equação (3.19) fazendo $h=0$ obtemos $C_{1}=y^{2}$ e para $h=-1$ concluimos $D_{1}=1-2 y+y^{2}=(1-y)^{2}$. Para $h=i(3.19)$ fica

$$
-1+2 i y+y^{2}=-2 A_{1}-B_{1}-(1-y)^{2}+i\left(-B_{1}+2 y^{2}\right),
$$

donde $B_{1}=2 y(y-1)$ e $A_{1}=-2 y(y-1)$. 
De modo análogo obtemos os valores de $A_{2}=2 y(y-1), B_{2}=-2 y(y-1), C_{2}=y^{2}$ e $D_{2}=(1-y)^{2}$. Observemos que $A_{1}=-A_{2}$ e $B_{2} /(h-1)=B_{1} /(1-h)$. Ao substituirmos as frações parciais em (3.18) e, escolhendo ho $\gamma=y+i t, 0<t<\infty$ quando $x=1$, temos

$$
\left.P\right|_{x=1}=\Re \int_{\gamma} B_{1}\left(\frac{1}{1+h}+\frac{1}{1-h}\right) \frac{d h}{i}-\Re \underbrace{\left\{i\left[-\frac{C_{1}+C_{2}}{h}-\frac{D_{1}}{1+h}+\frac{D_{2}}{1-h}\right]_{y}^{\infty}\right\}}_{=0}
$$

Logo,

$$
\begin{aligned}
\left.P\right|_{x=1} & =\Re\left\{B_{1} \int_{0}^{\infty}\left[\frac{1}{1+y+i t}+\frac{1}{1-y-i t}\right] d t\right\} \\
& =B_{1} \Re \int_{0}^{\infty}\left[\frac{1+y-i t}{(1+y)^{2}+t^{2}}+\frac{1-y-i t}{(1-y)^{2}+t^{2}}\right] d t \\
& =B_{1} \int_{0}^{\infty}\left[\frac{1+y}{(1+y)^{2}+t^{2}}+\frac{1-y}{(1-y)^{2}+t^{2}}\right] \\
& =B_{1}\left[\arctan \left(\frac{t}{1+y}\right)+\arctan \left(\frac{t}{1-y}\right)\right]_{0}^{\infty} \\
& =2 y(y-1) \pi=2 \pi y^{2}-2 \pi y .
\end{aligned}
$$

Ou seja, nesse caso temos a equação explícita da parábola $P(n, 1, y)$ :

$$
P(n, 1, y)=2 \pi y^{2}-2 \pi y
$$

donde concluímos $\left.a\right|_{x=1}=2 \pi,\left.b\right|_{x=1}=-2 \pi$ e $\left.c\right|_{x=1}=0$ (esta última já havia sido mostrada no item anterior desta proposição). Logo $1+\left.\frac{b+c}{a}\right|_{x=1}=0$.

(f) Do item acima segue-se que $\left.(a+b+c)\right|_{x=1}=0$. Lembrando que $\left.P\right|_{y=1}=a+b+c$ temos de (3.18) que

$$
a+b+c=\left.P\right|_{x=1}=\Re \int_{\gamma}\left(\tilde{F}+\tilde{F}^{-1}\right) \frac{d h}{i h^{2}},
$$

onde $\tilde{F}=\left(\frac{1+h}{1-h}\right) F=\left(\frac{x-h}{x+h}\right)^{1-\frac{1}{n}}\left(\frac{1+h}{1-h}\right)^{1-\frac{1}{n}}$.

As funções $\tilde{F}$ e $\tilde{F}^{-1}$ satisfazem as mesmas relações que $F$ e $F^{-1}$ quando derivadas em relação a $x$, pois o termo que depende de $x$ para $F$ e $\tilde{F}$ é o mesmo. Logo,

$$
\frac{\partial \tilde{F}}{\partial x}=2(1-1 / n) \frac{h}{x^{2}-h^{2}} \tilde{F} \mathrm{e} \frac{\partial \tilde{F}^{-1}}{\partial x}=-2(1-1 / n) \frac{h}{x^{2}-h^{2}} \tilde{F}^{-1}
$$


Portanto temos

$$
\frac{\partial}{\partial x}(a+b+c)=2(1-1 / n) \Re \int_{\gamma}\left(\tilde{F}-\tilde{F}^{-1}\right) \frac{d h}{x^{2}-h^{2}} .
$$

Como procedemos na demonstração do item (c), mostraremos que $\frac{\partial}{\partial x}(a+b+$ $c)>0$. Temos que $\lim _{h \rightarrow 0} \frac{\tilde{F}-\tilde{F}^{-1}}{h}=\frac{4(n-1)(x-1)}{n x}<\infty$, logo o integrando é analítico em $h=0$. Podemos considerar $\gamma$ tal que $h \circ \gamma=i t, 0<t<\infty$ e então

$$
\frac{\partial}{\partial x}(a+b+c)=2(1-1 / n) \Re \int_{0}^{\infty} \frac{\left(\tilde{F}-\tilde{F}^{-1}\right) \circ h(t)}{i t} \frac{d t}{x^{2}+t^{2}} .
$$

Do mesmo modo que ocorre com $F$ temos que $\tilde{F}-\tilde{F}^{-1}=2 i \Im \tilde{F}$, a qual é positiva pois agora $\Im F^{1-1 / n}=\frac{x^{2}-1}{x^{2}+1}>0 \Rightarrow \Im F>0$. Portanto o integrando acima é positivo, mostrando que $\frac{\partial}{\partial x}(a+b+c)>0$.

A proposição a seguir combinada com a anterior mostrará que o problema dos períodos tem soluções. Para tanto definimos a estensão de $F(x, h)$ para $x=\infty$ :

$$
F(\infty, h):=\left(\frac{1-h}{1+h}\right)^{\frac{1}{n}}
$$

e então $P(n, \infty, y), a(n, \infty, y), b(n, \infty, y)$ e $c(n, \infty, y)$ ficam definidas como em (3.8), (3.9), (3.10) e (3.11), respectivamente.

Proposição 3.3.2. Valem as seguintes afirmações:

(a) $P(n, \infty, y)$ é contínua em $x=\infty$;

(b) $a, b$ e c são contínuas em $x=\infty$;

(c) $P(n, \infty, y)>0, \forall y \in(0,1)$.

\section{Demonstração:}

(a) Vamos incialmente fazer algumas estimativas para a função $h$. Seja $0<\varepsilon<1$. Então

$$
\begin{aligned}
|h| \leq \frac{\varepsilon}{2+\varepsilon} x & \Rightarrow 2|h| \leq \varepsilon(|x|-|h|) \leq \varepsilon|x+h| \\
& \Rightarrow\left|\frac{2 h}{x+h}\right| \leq \varepsilon \\
& \Rightarrow\left|1-\frac{x-h}{x+h}\right| \leq \varepsilon \\
& \Rightarrow 1-\varepsilon \leq\left|\frac{x-h}{x+h}\right| \leq 1+\varepsilon .
\end{aligned}
$$


E portanto, se $|h| \leq \varepsilon /(2+\varepsilon)$, também temos

$$
\frac{1}{1+\varepsilon} \leq\left|\frac{x+h}{x-h}\right| \leq \frac{1}{1-\varepsilon}
$$

Como $0<\varepsilon<1$ temos $1-\varepsilon<1 \Rightarrow 1-\varepsilon<\sqrt[n]{1-\varepsilon}<\sqrt[n]{1+\varepsilon}<1+\varepsilon$ e das estimativas acima temos

$1-\frac{2 \varepsilon}{1-\varepsilon}<1-\frac{2 \varepsilon}{1+\varepsilon}=\frac{1-\varepsilon}{1+\varepsilon}<\frac{1-\varepsilon}{\sqrt[n]{1+\varepsilon}} \leq\left|\frac{x-h}{x+h}\right|^{\frac{n-1}{n}} \leq \frac{1+\varepsilon}{\sqrt[n]{1-\varepsilon}}<1+\frac{2 \varepsilon}{1-\varepsilon}$ donde

$$
1-\frac{2 \varepsilon}{1-\varepsilon} \leq\left|\frac{x+h}{x-h}\right|^{\frac{n-1}{n}}<1+\frac{2 \varepsilon}{1-\varepsilon}
$$

Então se $\nu:=\frac{2 \varepsilon}{1-\varepsilon}$ temos

$$
\left|\left(\frac{x-h}{x+h}\right)^{\frac{n-1}{n}}-1\right|<\nu \mathrm{e}\left|\left(\frac{x+h}{x-h}\right)^{\frac{n-1}{n}}-1\right|<\nu .
$$

Sejam $M=M(x, \varepsilon):=\frac{\varepsilon}{2+\varepsilon} x$ e $\gamma$ uma curva no "birdcage" tal que $h \circ \gamma=$ $y+i t, 0<t<\infty$. Sejam ainda $\gamma_{1}$ e $\gamma_{2}$ curvas tais que $\gamma=\gamma_{1}+\gamma_{2}$ com $\left|h \circ \gamma_{1}\right| \leq M$ e $\left|h \circ \gamma_{2}\right| \geq M$.

As transformações de Möbius $\frac{x-h}{x+h}$ e $\frac{x+h}{x-h}$ levam $h \circ \gamma$ em circunferências cujos raios são menores que $\frac{x+y}{x-y}$. E, se $x \geq 3 y$ temos $\frac{x+y}{x-y} \leq 2$. Então sobre $\gamma_{2}$ temos

$$
\mid \begin{aligned}
& \left(\frac{x-h}{x+h}\right)^{\frac{n-1}{n}}-1 \mid<2^{\frac{n-1}{n}}+1<3 \quad \text { e, do mesmo modo } \\
& \left(\frac{x+h}{x-h}\right)^{\frac{n-1}{n}}-1 \mid<2^{\frac{n-1}{n}}+1<3
\end{aligned}
$$

Fazendo $x=1$ nas observações acima obtemos

$$
\left|\frac{1-h}{1+h}\right|^{\frac{1}{n}}<\left(\frac{1+y}{1-y}\right)^{\frac{1}{n}}<\frac{1+y}{1-y}
$$

Se

$$
\omega:=\frac{|h+y|^{2}\left|F-\left(\frac{1-h}{1+h}\right)^{\frac{1}{n}}\right|+|h-y|^{2}\left|F^{-1}-\left(\frac{1+h}{1-h}\right)^{\frac{1}{n}}\right|}{|h|^{2}\left|1-h^{2}\right|}|d h|
$$


então de (3.20), (3.21) e (3.22) temos

$$
\begin{aligned}
|P(n, x, y)-P(n, \infty, y)| & \leq \int_{\gamma_{1}} \omega+\int_{\gamma_{2}} \omega \\
& <\nu \frac{1+y}{1-y} \int_{\gamma_{1}} \frac{|h+y|^{2}+|h-y|^{2}}{|h|^{2}\left|1-h^{2}\right|}|d h| \\
& +3 \frac{1+y}{1-y} \int_{\gamma_{2}} \frac{|h+y|^{2}+|h-y|^{2}}{|h|^{2}\left|1-h^{2}\right|}|d h|
\end{aligned}
$$

Definindo $I_{k}=\frac{1+y}{1-y} \int_{\gamma_{k}} \frac{|h+y|^{2}+|h-y|^{2}}{|h|^{2}\left|1-h^{2}\right|}|d h|, k=1,2$ temos

$$
|P(n, x, y)-P(n, \infty, y)|<\nu I_{1}+3 I_{2}
$$

As transformações de Möbius $\frac{h \pm y}{h \pm 1}$ levam as curvas nas integrais $I_{k}$ acima em semi-círculos cujos raios são menores que $\frac{1+y}{1-y}$, donde

$$
\left|\frac{(h \pm y)^{2}}{h^{2}-1}\right| \leq\left(\frac{1+y}{1-y}\right)^{2}
$$

Portanto,

$$
\begin{aligned}
& I_{1} \leq\left(\frac{1+y}{1-y}\right)^{3} \int_{h \circ \gamma_{1}} \frac{d t}{y^{2}+t^{2}} \mathrm{e} \\
& I_{2} \leq\left(\frac{1+y}{1-y}\right)^{3} \int_{h \circ \gamma_{2}} \frac{d t}{t^{2}} .
\end{aligned}
$$

Agora, $\left|h \circ \gamma_{1}\right|=|y+i t| \leq M \Rightarrow t \leq M+y$ e $\left|h \circ \gamma_{2}\right|=|y+i t| \geq M \Rightarrow t \geq M-y$. Então se $0<\varepsilon<1, M<1$ e $x>3 y$ temos

$$
\begin{aligned}
& I_{1} \leq\left(\frac{1+y}{1-y}\right)^{3} \frac{1}{y} \arctan \left(\frac{M+y}{y}\right) \leq\left(\frac{1+y}{1-y}\right)^{3} \frac{\pi}{y} \mathrm{e} \\
& I_{2} \leq\left(\frac{1+y}{1-y}\right)^{3} \frac{1}{M-y} .
\end{aligned}
$$

Das majorações acima aplicadas em (3.23) temos

$$
|P(n, x, y)-P(n, \infty, y)|<\left(\frac{1+y}{1-y}\right)^{3} \frac{1}{y}\left(\nu \pi+\frac{3 y}{M-y}\right) .
$$


Como $M=\frac{\varepsilon}{2+\varepsilon}$, se $x \geq \frac{2+\varepsilon}{\varepsilon^{2}}$ e $\varepsilon \leq 1 / 2$ então $M>1 / \varepsilon \Rightarrow \frac{1}{M-y}<2 \varepsilon$ e $\nu=\frac{2 \varepsilon}{1-\varepsilon}<4 \varepsilon$. Definindo $k:=\left(\frac{1+y}{1-y}\right)^{3} \frac{1}{y}(4 \pi+6 y)$ e considerando $\varepsilon=$ $\min \{1 / 3, \tilde{\varepsilon} / k\}, \operatorname{com} \tilde{\varepsilon}>0$ arbitrário temos que

dado $\tilde{\varepsilon}>0, \exists \delta=\frac{2+\varepsilon}{\varepsilon^{2}}$ tal que $x>\delta \Rightarrow|P(n, x, y)-P(n, \infty, y)|<\tilde{\varepsilon}$, ou seja, $P(n, x, y)$ é contínua em $x=\infty$.

(b) Acabamos de provar que dado $\tilde{\varepsilon}>0$ existe $\delta>0$ tal que se $x>\delta$ então $|P(n, x, y)-P(n, \infty, y)|<\tilde{\varepsilon}$. Em termos de sequências isto quer dizer que dada $\left\{r_{k}\right\}_{k \in \mathbb{N}}$ sequência de números reais tal que $\lim _{k \rightarrow \infty} r_{k}=\infty$ temos que

$$
\lim _{k \rightarrow \infty} P\left(n, r_{k}, y\right)=P(n, \infty, y)
$$

ou seja, a sequência $\left\{P\left(n, r_{k}, y\right)\right\}$ é convergente e portanto de Cauchy, ie, dado $\tilde{\varepsilon}>0, \exists N \in \mathbb{N}$ tal que se $m_{1}, m_{2} \geq N$ então

$$
\left|P\left(n, r_{m_{1}}, y\right)-P\left(n, r_{m_{2}}, y\right)\right|<\tilde{\varepsilon}
$$

Podemos aqui supor que a sequência $\left\{r_{k}\right\}$ é crescente. Podemos ainda reescrever a equação 3.24 usando a equação de $P(n, x, y)$ em termos dos coeficientes $a, b$ e $c$, abusando da notação, do seguinte modo:

$$
\begin{gathered}
\mid\left[(a+b+c)\left(r_{m_{2}}\right)-(a+b+c)\left(r_{m_{1}}\right)\right] y^{2}+ \\
{\left[b\left(r_{m_{2}}\right)-b\left(r_{m_{1}}\right)\right]\left(y-y^{2}\right)+} \\
{\left[c\left(r_{m_{2}}\right)-c\left(r_{m_{1}}\right)\right]\left(1-y^{2}\right) \mid<\tilde{\varepsilon} .}
\end{gathered}
$$

Dos itens (a), (d) e (f) da proposição 3.3.1 segue-se que cada parcela da inequação acima é positiva, pois $y \in(0,1)$. Assim

$$
\begin{gathered}
{\left[(a+b+c)\left(r_{m_{2}}\right)-(a+b+c)\left(r_{m_{1}}\right)\right] y^{2}+} \\
{\left[b\left(r_{m_{2}}\right)-b\left(r_{m_{1}}\right)\right]\left(y-y^{2}\right)+} \\
{\left[c\left(r_{m_{2}}\right)-c\left(r_{m_{1}}\right)\right]\left(1-y^{2}\right)<\tilde{\varepsilon}}
\end{gathered}
$$

$\forall y \in(0,1)$. Daí temos que

$$
\left\{\begin{array}{l}
(a+b+c)\left(r_{m_{2}}\right)-(a+b+c)\left(r_{m_{1}}\right)<\tilde{\varepsilon} / y^{2} \\
b\left(r_{m_{2}}\right)-b\left(r_{m_{1}}\right)<\tilde{\varepsilon} /\left(y-y^{2}\right) \mathrm{e} \\
c\left(r_{m_{2}}\right)-c\left(r_{m_{1}}\right)<\tilde{\varepsilon} /\left(1-y^{2}\right) .
\end{array}\right.
$$


Disto temos que $a\left(r_{k}\right), b\left(r_{k}\right)$ e $c\left(r_{k}\right)$ são sequências de Cauchy. Assim, para $\hat{a}:=\lim _{k \rightarrow \infty} a\left(r_{k}\right), \hat{b}:=\lim _{k \rightarrow \infty} b\left(r_{k}\right)$ e $\hat{c}:=\lim _{k \rightarrow \infty} c\left(r_{k}\right)$ temos que

$$
P(n, \infty, y)=\hat{a} y^{2}+\hat{b} y+\hat{c}
$$

donde $\hat{a}=a(n, \infty, y), \hat{b}=b(n, \infty, y)$ e $\hat{c}=c(n, \infty, y)$ para todo $y \in(0,1)$. Logo as funções $a, b$ e $c$ são contínuas em $x=\infty$.

(c) Podemos escrever, quando $x=\infty$, a equação 3.2 como

$$
w^{n}=\frac{1-h}{1+h}
$$

O gênero da superfície de Riemann dada pela equação algébrica acima é $g=$ $\frac{2(n-1)}{2}-n+1=0$, ou seja, uma esfera (lembrando que 1 e -1 são pontos de ramificação de ordem $n-1$ ). Portanto, quando $x=\infty$ temos a imersão mínima de uma esfera com $n+2$ fins catenoidais com as mesmas simetrias euclideanas do "birdcage-catenóide". A figura 3.10 representa esta superfície.

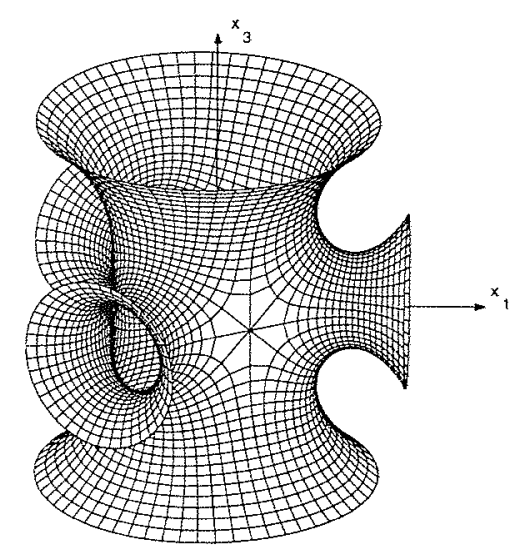

Figura 3.10: 5-nóide.

Vamos deduzir os dados de Weierstrass para esta superfície baseados em sua geometria e no tipo de análise feita para o "birdcage-catenóide" e para os exemplos do capítulo anterior. Como queremos que a superfície tenha dois fins catenoidais verticais e uma simetria rotacional de $360 / n$ graus vamos considerar uma fatia do catenóide

$$
z^{n}=\frac{1-\tilde{h}}{1+\tilde{h}}
$$


Trocamos aqui as notações para evitar confusão com os dados de Weierstrass do bridcage-catenóide. Queremos ainda que a superfície tenha um fim catenoidal horizontal em torno do eixo $x_{1}$ (correspondente ao valor $\tilde{h}=0$ ) e com isso, além do crescimento logarítmico na primeira coordenada, devemos ter um valor $y \in$ $(0,1)$ onde o vetor normal é vertical. Assim, se $(G, d \tilde{H})$ é o par de Weierstrass de $(n+2)$-nóide, podemos escrever

$$
G=z \frac{\tilde{h}+y}{\tilde{h}-y} .
$$

Para encontrar $d \tilde{H}$, observemos que ela deve ter pólos simples nos fins verticais e um pólo duplo no fim horizontal quando quando $h=0$, dando o mesmo tipo de crescimento nas duas últimas coordenadas da representação de Weierstrass, enquanto temos crescimento logarítmico na primeria coordenada. A diferencial $d \tilde{H}$ deve ter zeros nos pontos regulares da superfície, onde o vetor normal é vertical (ie, $h= \pm y$ ). Então temos que

$$
d \tilde{H}=\frac{\tilde{h}^{2}-y^{2}}{h^{2}} \frac{d \tilde{h}}{\tilde{h}^{2}-1} .
$$

Em princípio os lados de (3.28) são apenas proporcionais, mas $\tilde{h}=i t, 0<t<\infty$ representa a curva na superfície contida no plano $x_{3}=0$, donde $d \tilde{H}$ deve ser imaginária pura ao longo desta curva e portanto a constante de proporção deve ser real, podendo ser escolhida unitária. Todas as simetrias requeridas para a superfície são satisfeitas pelos dados de (3.27) e (3.28). Os períodos se anulam devido às simetrias existentes nos fins catenoidais.

De (3.26) temos $\tilde{h}=\frac{1-z^{n}}{1+z^{n}}$. Definindo $\lambda=\frac{1+y}{1-y}$, podemos reescrever $(3.27) \mathrm{e}$ (3.28) como

$$
\begin{aligned}
G & =z \frac{z^{n}-\lambda}{\lambda z^{n}-1} \mathrm{e} \\
d \tilde{H} & =\frac{\left(z^{n}-\lambda\right)\left(\lambda^{-1}-z^{n}\right)}{z\left(z^{n}-1\right)^{2}} d z=\frac{\lambda+\lambda^{-1}-\left(z^{n}+z^{-n}\right)}{z^{n}+z^{-n}-2} \frac{d z}{z}
\end{aligned}
$$

Observe que descartamos uma constante em (3.30) que corresponde a uma dilatação da superfície em $\mathbb{R}^{3}$. A figura 3.11 representa os zeros e pólos de $G$ e $d \tilde{H}$ na esfera.

Da figura 3.11, concluímos que se verificam as seguintes expressões: 


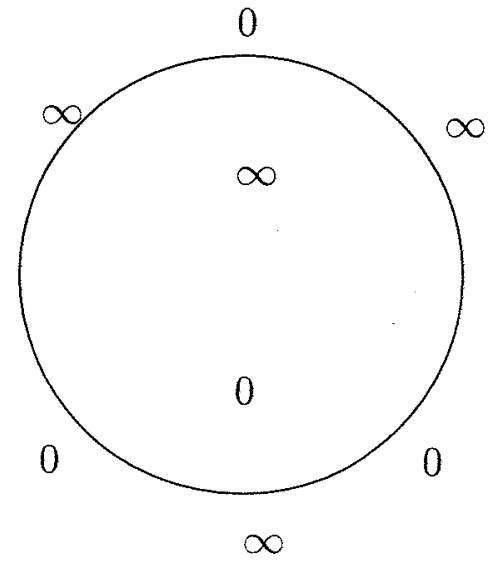

$G$

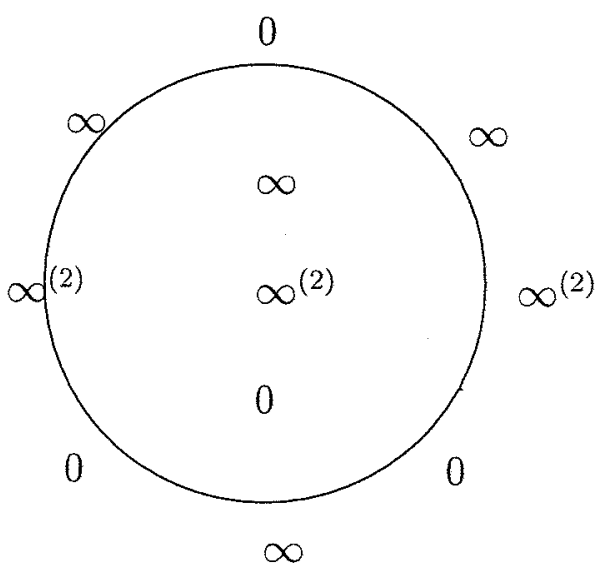

$d \tilde{H}$

Figura 3.11: Zeros e pólos dos dados de Weierstrass do $n+2$-nóide.

$$
\begin{aligned}
\operatorname{deg}(G) & =\gamma+r-1=0+n+2-1=n+1, \\
\operatorname{deg}(d \tilde{H}) & =-\chi\left(S^{2}\right)=-2
\end{aligned}
$$

Considere agora as curvas $z_{1}(t)=t, z_{2}(t)=t e^{-i \frac{\pi}{n}}$ e $z_{3}(t)=e^{i \frac{\pi}{n} t}, 0 \leq t \leq 1$. Suas imagens pela representação de Weierstrass são levadas em geodésicas planas do $(n+2)$-nóide, pois $\left.\frac{d G}{G} d \tilde{H}\right|_{z_{i}} \in \mathbb{R}, i=1,2,3$. As imagens de $z_{1}$ e $z_{2}$ por $G$ são retas que formam ângulo de $180 / n$ em $0 \in \mathbb{C}$ (observe que $z=0$ correponde ao fim catenoidal superior). Logo, as geodésicas planas correspondentes a $z_{1}$ e $z_{2}$ estão em planos verticais não paralelos formando ângulo de $180 / n$ graus, pois $z=0$ não é ponto de ramificação de $G$. Se o $n+2$ nóide estiver posicionado como na figura 3.10 então esses planos são $x_{1}=0$ e $x_{2}=-\tan (\pi / n) x_{1}$. A terceira geodésica, correspondente à curva $z_{3}$, está contida no plano $x_{3}=0$.

Observando que $G\left(z_{1}\right) \subset \mathbb{R}, G\left(z_{2}\right) \subset\left\{t e^{-i \frac{\pi}{n} t}\right\}$ e $G\left(z_{3}\right) \subset S^{1}$ com extremos $G\left(z_{1}(0)\right)=G\left(z_{2}(1)\right), G\left(z_{1}(1)\right)=G\left(z_{3}(0)\right)$ e $G\left(z_{2}(0)\right)=G\left(z_{3}(1)\right)$ temos a figura 3.12 que mostra o interior da região limitada por $z_{1}, z_{2}$ e $z_{3}$ e sua imagem por G.

Considerando a segunda coordenada da representação de Weierstrass do $(n+2)$ nóide temos

$$
\varphi_{2}=\frac{i}{2}\left(G+\frac{1}{G}\right) d \tilde{H}=\frac{-i}{z\left(z^{n}-1\right)^{2}}\left[\frac{z}{\lambda}\left(z^{n}-\lambda\right)^{2}+\frac{\lambda}{z}\left(z^{n}-\lambda^{-1}\right)^{2}\right]
$$




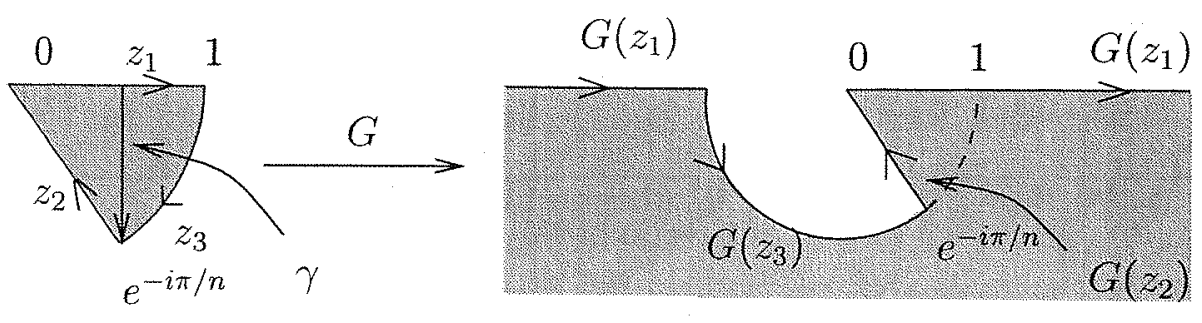

Figura 3.12: Setor circular e sua imagem por $G$.

$\mathrm{Na}$ figura 3.12 a curva $\gamma$ representa a menor geodésica ligando $\tilde{h}=y$ (ou seja, $z=\sqrt[n]{\lambda^{-1}}$ ) até $z=e^{-i \pi / n}$. A imagem de $\gamma$ está contida no interior do setor circular da figura 3.12 pois caso contrário as simetrias com relação a $z_{i}, i=1,2,3$ no $(n+2)$-nóide a confinariam no setor. Considere agora

$$
x_{2}(t)=\Re \int_{0}^{t} \varphi_{2}(\gamma(\zeta)) \gamma^{\prime}(\zeta) d \zeta, 0 \leq t \leq 1
$$

Daí, $x_{2}(0)=0, x_{2}(1)=\int_{\gamma} \varphi_{2}$ e $x_{2}^{\prime}(t)=\Re\left[\varphi_{2}(\gamma(t)) \gamma^{\prime}(t)\right]$.

Substituindo $z=\sqrt[n]{\lambda^{-1}}$ em (3.31) temos que $\left.\varphi_{2}\right|_{z=\sqrt[n]{\lambda^{-1}}} \in i \mathbb{R}_{-}^{*}$. Mas da figura $3.12 \Im\left(\gamma^{\prime}(0)\right)$ é negativa. Então

$$
x_{2}^{\prime}(0)<0
$$

Seja $\Gamma$ a composição de $\gamma$ com a imersão mínima dada por $(G, d \tilde{H})$. Então $x_{2}^{\prime \prime}(t)$ é a segunda componente do vetor normal $\Gamma^{\prime \prime}(t)$ na superfície. O módulo desse vetor normal é dado por $\left|\Gamma^{\prime \prime}(t)\right|$. Vamos agora mostrar que $\left|\Gamma^{\prime \prime}(t)\right|$ nunca se anula para $0<t<1$.

Das figuras 3.11 e 3.12 vemos que $G$ e $d H$ não têm zeros ou pólos no interior do setor circular. Além disso, $d G$ não tem zeros nessa região, pois caso contrário $d G$ teria pelo menos $4 n$ zeros em toda a superfície devido à simetria rotacional, mas como $d G$ tem $2 n+2$ pólos no total teríamos então $\operatorname{deg}(d G)=2 n-2$, contradição, uma vez que $\operatorname{deg}(d G)=-2$. Disto concluímos ainda que $d G$ deve ter $2 n$ zeros, que estão sobre as curvas $z_{1}, z_{2}$ ou $z_{3}$ e suas imagens pelas simetrias do $(n+2)$-nóide. Assim, a curvatura gaussina $K$ (veja a expressão de $K$ em (1.4.15) não se anula no interior do setor. Portanto $\left|\Gamma^{\prime \prime}(t)\right|$ é positivo para $0<t<1$.

Disto segue-se que $\left|x_{2}^{\prime \prime}(t)\right| \neq 0,0<t<1$, donde $x_{2}^{\prime \prime}(t)<0,0<t<1$ ou $x_{2}^{\prime \prime}(t)>0,0<t<1$. 
Se $x_{2}^{\prime \prime}(t)<0,0<t<1$ então (3.32) implica que $x_{2}^{\prime}(t)<0,0<t<1$ e portanto de (3.6) temos $P(n, \infty, y)=-2 x_{2}(1)>0$.

Se provarmos que $x_{2}^{\prime}(1)<0$, obteremos o mesmo resultado no caso em que $x_{2}^{\prime \prime}(t)>0$.

Da figura (3.12) vemos que $-\frac{\pi}{n}-\frac{\pi}{2}<\arg \left(\gamma^{\prime}(1)\right)<-\frac{\pi}{n}$. Além disso

$$
\begin{aligned}
\varphi_{2}(\gamma(1)) & =\frac{i}{2}\left(e^{-i \frac{\pi}{n}} \frac{-1-\lambda}{-\lambda-1}+e^{i \frac{\pi}{n}} \frac{-\lambda-1}{-1-\lambda}\right) \frac{(-1-\lambda)\left(\lambda^{-1}+1\right)}{e^{-i \frac{\pi}{n}} 4} \\
& =\frac{i}{8}\left(1+e^{2 i \frac{\pi}{n}}\right)\left(-\lambda^{-1}-1-1-\lambda\right) \\
& =-\frac{i}{8} \frac{(\lambda+1)^{2}}{\lambda}\left(1+e^{2 i \frac{\pi}{n}}\right) .
\end{aligned}
$$

E portanto $-\pi<\arg \left(\varphi_{2}(\gamma(1)) \gamma^{\prime}(1)\right)<-\frac{\pi}{2} \Rightarrow x_{2}^{\prime}(1)<0$. E então com $x_{2}^{\prime}(1)<$ 0 e $x_{2}^{\prime \prime}(t)>0$ temos que $x_{2}^{\prime}(t)$ não assume máximo para $0<t<1$, e então $x_{2}(t)<0,0<t<1$, donde $P(n, \infty, y)=-2 x_{2}(1)>0$.

Com as duas proposições acima podemos concluir que se $y_{1}(x)$ e $y_{2}(x)$ são as raízes de $P(n, x, y)=0$ com

$$
y_{1}(x)=\frac{-b(n, x)+\sqrt{\Delta(n, x)}}{2 a(n, x)} \text { e } y_{2}(x)=\frac{-b(n, x)-\sqrt{\Delta(n, x)}}{2 a(n, x)},
$$

com $\Delta(n, x)=b^{2}(n, x)-4 a(n, x) c(n, x)$ então $y_{1}$ e $y_{2}$ são funções contínuas de $x$ e do item (e) da proposição 3.3.1 omitindo $n$ do argumento das funções vem que

1. $P(n, 1,0)=c(1)=0$.

2. $P(n, 1,1)=(a+b+c)(1)=0 \Rightarrow b(1)=-a(1)$.

3. $\lim _{x \rightarrow 1} y_{1}(x)=\frac{-b(1)+\sqrt{\Delta(1)}}{-2 b(1)}=0$.

4. $\lim _{x \rightarrow 1} y_{2}(x)=\frac{-b(1)+\sqrt{\Delta(1)}}{-2 b(1)}=1$.

As funções $y_{1}$ e $y_{2}$ acima são respectivamente crescente e decrescente com $x$, pois se $\tilde{b}:=b / a$ e $\tilde{c}:=c / a$, podemos escrever $2 y_{1}=\tilde{b}+\sqrt{\tilde{\Delta}}$ e $2 y_{2}=\tilde{b}-\sqrt{\tilde{\Delta}}$, com $\tilde{\Delta}=\tilde{b}^{2}-4 \tilde{c}$. Logo,

$$
\frac{\partial \tilde{\Delta}}{\partial x}=\frac{\partial}{\partial x}\left(\tilde{b}^{2}-4 \tilde{c}\right)=2 \tilde{b} \tilde{b}^{\prime}-4 \tilde{c}^{\prime}=2 \tilde{b}\left(\tilde{b}^{\prime}+\tilde{c}^{\prime}\right)-(2 \tilde{b}+4) \tilde{c}^{\prime} .
$$


Mas segue-se dos itens (a), (c) e (d) da proposição 3.3.1 que

$$
\tilde{c}^{\prime}>0, \tilde{b}^{\prime}>0 \text { e } \tilde{b}<0 .
$$

Do item (b) da mesma proposição temos

$$
-b<2 a \Rightarrow-\tilde{b}<2 \Rightarrow-2 \tilde{b}-4<0 .
$$

De (3.33) e (3.34) temos que

$$
\frac{\partial \tilde{\Delta}}{\partial x}<0
$$

donde concluimos que $\frac{\partial y_{1}}{\partial x}>0$ e $\frac{\partial y_{2}}{\partial x}<0$.

Para mostrar que existe $x_{n}, 1<x_{n}<\infty$ tal que o problema dos períodos tem uma única solução basta observar da proposição 3.3 .2 e das considerações acima que

$$
\begin{aligned}
P(n, \infty, y)>0 & \Rightarrow \Delta(\infty)<0 \mathrm{e} \\
P(n, 1,0)=P(n, 1,1)=0 & \Rightarrow \Delta(1)>0 .
\end{aligned}
$$

Logo existe $x_{n} \in(1, \infty)$ tal que $\Delta\left(x_{n}\right)=0$. Como as funções $y_{1}$ e $y_{2}$ são respectivamente crescente e decrescente em $x$, temos que esse $x_{n}$ é único. Se $1<x<x_{n}$ temos $\Delta(x)>0$, e portanto duas soluções para o problema dos períodos. Se $x_{n}<x<\infty$ então $\Delta(x)<0$ e não há solução para o problema dos períodos. A figura 3.13 representa cada situação
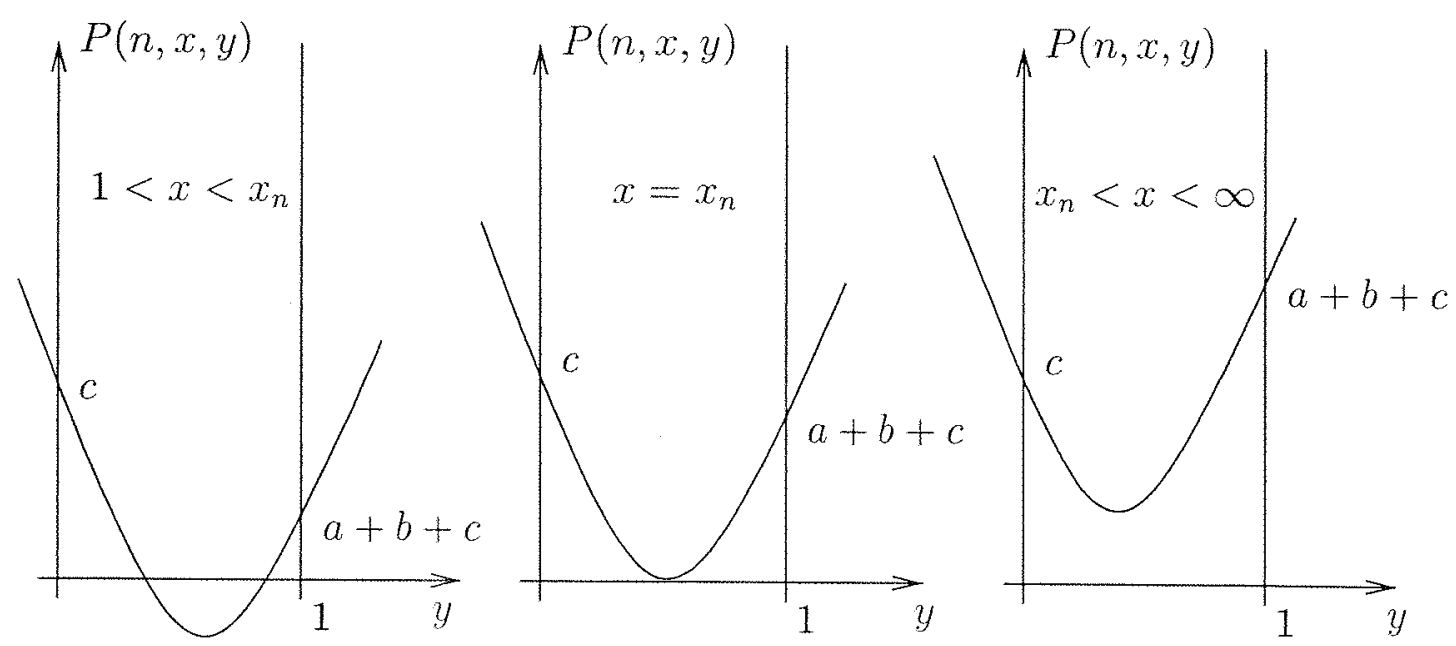

Figura 3.13: Representações de $P(n, x, y)$. 


\section{Referências Bibliográficas}

[1] V. Ramos Batista. Noncongruent minimal surfaces with the same symmetries and conformal structure. Tohoku Mathematical Journal, To appear.

[2] L. Bers. Riemann surfaces. New York University, 1957-1958.

[3] J. B. Conway. Functions of one complex variable. Springer-Verlag, 1975.

[4] C. J. da Costa. Funções elípiticas, algébricas e superfícies mínimas. $18^{\circ}$ Colóquio Brasileiro de Matemática. IMPA, 1991.

[5] F.J. López e A. Ros. On embedded complete minimal surfaces of genus zero. Journal of Differential Geometry, 33:293-300, 1991.

[6] M. Callaham e D. Hoffman e W. Meeks. Embedded minimal surfaces with an infinite number of ends. Inventiones Mathematicae, 96:459-505, 1989.

[7] L. Jorge e W. H. Meeks III. The topology of complete minimal surfaces of finite total gaussian curvature. Topology, 22:203-221, 1983.

[8] O. Forster. Lectures on Riemann Surfaces. Springer-Verlag, 1981.

[9] H. Fujimoto. Value distribution theory of the Gauss Map of Minimal Surfaces in $\mathbb{R}^{m}$. Friedrich Vieweg \& Sohn, 1993.

[10] H. Karcher. Construction of minimal surfaces. Technical report, Tokyo University, 1989.

[11] W. S. Massey. Algebraic Topology: An introduction. Springer-Verlag, New York, 1967.

[12] R. Osserman. A survey of minimal surfaces. Dover, $2^{\text {nd }}$ ed., 1986.

[13] R. Schoen. Uniqueness, simetry and embeddeness of minimal surfaces. Journal of Differential Geometry, 18:791-809, 1983. 
[14] M. Spivak. Comprehensive introduction to differential geometry. Library of Congress, 1975.

[15] G. Springer. Introduction to Riemann Surfaces. Chelsea Publishing Co., New York, $2^{\text {nd }}$ ed., 1981.

[16] M. Wohlgemuth. Higher genus minimal surfaces by growing handles out of a catenoid. Manuscripta Math., 70:397-428, 1991.

[17] M. Wohlgemuth. Minimal surfaces of higher genus with finite total curvature. Archive for Rational Mechanics and Analysis, 137:1-25, 1997.

[18] Antonio Chávez Zeña. Torres de selas simétricas do tipo scherk. Tese de mestrado IME-USP, 2002. 